\title{
Strong isospin symmetry breaking in light scalar meson production
}

\author{
N. N. Achasov* and G. N. Shestakov ${ }^{\dagger}$ \\ Laboratory of Theoretical Physics, Sobolev Institute for Mathematics, \\ Siberian Branch of the Russian Academy of Sciences, \\ prosp. Akademika Koptyuga 4, 630090 Novosibirsk, Russian Federation
}

\begin{abstract}
Isospin symmetry breaking is discussed as a tool for studying the nature and production mechanisms of light scalar mesons. We are concerned with isospin breaking effects with an amplitude $\sim \sqrt{m_{d}-m_{u}}$ (instead of the usual $\sim m_{d}-m_{u}$ ), where $m_{u}$ and $m_{d}$ are the $u$ and $d$ quark masses, whose magnitude and phase vary with energy in a resonance-like way characteristic of the $K \bar{K}$ threshold region. We consider a variety of reactions that can experimentally reveal (or have revealed) the mixing of $a_{0}^{0}(980)$ and $f_{0}(980)$ resonances that breaks the isotopic invariance due to the mass difference between $K^{+}$and $K^{0}$ mesons. Experimental results on the search for $a_{0}^{0}(980)-f_{0}(980)$ mixing in $f_{1}(1285) \rightarrow f_{0}(980) \pi^{0} \rightarrow \pi^{+} \pi^{-} \pi^{0}$ and $\eta(1405) \rightarrow f_{0}(980) \pi^{0} \rightarrow \pi^{+} \pi^{-} \pi^{0}$ decays suggest a broader perspective on the isotopic symmetry breaking effects due to the $K^{+}$and $K^{0}$ mass difference. It has become clear that not only the $a_{0}^{0}(980)-f_{0}(980)$ mixing but also any mechanism producing $K \bar{K}$ pairs with a definite isospin in an $\mathrm{S}$ wave gives rise to such effects, thus suggesting a new tool for studying the nature and production mechanisms of light scalars. Of particular interest is the case of a large isotopic symmetry breaking in the $\eta(1405) \rightarrow f_{0}(980) \pi^{0} \rightarrow \pi^{+} \pi^{-} \pi^{0}$ decay due to the occurrence of anomalous Landau thresholds (logarithmic triangle singularities), i.e., due to the $\eta(1405) \rightarrow\left(K^{*} \bar{K}+\bar{K}^{*} K\right) \rightarrow\left(K^{+} K^{-}+K^{0} \bar{K}^{0}\right) \pi^{0} \rightarrow f_{0}(980) \pi^{0} \rightarrow \pi^{+} \pi^{-} \pi^{0}$ transition (where it is of fundamental importance that the $K^{*}$ meson has a finite width).
\end{abstract}

Keywords: physics of light scalar mesons, isospin symmetry breaking, resonance mixing, reaction mechanisms, experimental investigations

\section{Introduction. Nature of scalar mesons}

2. The $a_{0}^{0}(980)-f_{0}(980)$ mixing as a threshold phenomenon. Amplitude and phase of the $a_{0}^{0}(980) \rightarrow\left(K^{+} K^{-}+K^{0} \bar{K}^{0}\right) \rightarrow f_{0}(980)$ transition. First search proposals

2.1. Peripheral reactions $\pi^{ \pm} N \rightarrow\left(a_{0}^{0}(980)\right.$, $\left.f_{0}(980)\right)(N, \Delta) \rightarrow \eta \pi^{0}(N, \Delta)$;

2.2. Reactions $\left(K^{-}, \bar{K}^{0}\right) N \rightarrow\left(f_{0}(980), a_{0}^{0}(980)\right)$ $(\Lambda, \Sigma, \Sigma(1385)) \rightarrow\left(\pi^{+} \pi^{-} / \eta \pi^{0}\right)(\Lambda, \Sigma, \Sigma(1385))$;

2.3. Reactions of $\bar{p} n$ annihilation at rest $\bar{p} n \rightarrow$ $\left(\pi^{-}, \rho^{-}\right) f_{0}(980) \rightarrow\left(\pi^{-}, \rho^{-}\right) \eta \pi^{0}$

2.4. Decay $f_{1}(1285) \rightarrow a_{0}^{0}(980) \pi^{0} \rightarrow 3 \pi$.

3. The $a_{0}^{0}(980)-f_{0}(980)$ mixing in polarization phenomena. Reaction $\pi^{-} p \uparrow \rightarrow \eta \pi^{0} n$

4. Detection of $a_{0}^{0}(980)-f_{0}(980)$ mixing

4.1. VES experiment in Protvino: the reaction $\pi^{-} N \rightarrow \pi^{-} f_{1}(1285) N \rightarrow \pi^{-} f_{0}(980) \pi^{0} N \rightarrow$ $\pi^{-} \pi^{+} \pi^{-} \pi^{0} N$

4.2. BESIII experiment in Beijing: reactions $\quad J / \psi \rightarrow \phi f_{0}(980) \rightarrow \phi a_{0}(980) \rightarrow \phi \eta \pi^{0}$ and $\chi_{c 1} \rightarrow a_{0}(980) \pi^{0} \rightarrow f_{0}(980) \pi^{0} \rightarrow \pi^{+} \pi^{-} \pi^{0} ;$

4.3. Data analysis

5. Strong violation of isotopic invariance according to BESIII data for the reactions $J / \psi \rightarrow \phi f_{1}(1285) \rightarrow \phi f_{0}(980) \pi^{0} \rightarrow \phi 3 \pi$ and

\footnotetext{
*E-mail: achasov@math.nsc.ru

$\dagger^{\dagger}$ E-mail: shestako@math.nsc.ru
}

$J / \psi \rightarrow \gamma \eta(1405) \rightarrow \gamma f_{0}(980) \pi^{0} \rightarrow \gamma 3 \pi$

5.1. Mechanisms of $f_{1}(1285) \rightarrow f_{0}(980) \pi^{0} \rightarrow 3 \pi$ decay;

5.2. Consistency condition;

5.3. $K \bar{K}$-loop mechanism of isotopic invariance violation in the decay $\eta(1405) \rightarrow f_{0}(980) \pi^{0} \rightarrow 3 \pi$ and the role of anomalous Landau thresholds

6. Manifestation of $a_{0}^{0}(980)-f_{0}(980)$ mixing in decays of charmed mesons

6.1. Decay $D_{s}^{+} \rightarrow \eta \pi^{0} \pi^{+}$;

6.2. Decays $D^{0} \rightarrow K_{S}^{0} \pi^{+} \pi^{-}$and $D^{0} \rightarrow K_{S}^{0} \eta \pi^{0}$

7. Bottomonium decay $\Upsilon(\mathbf{1 0 8 6 0}) \rightarrow$ $\Upsilon(1 S) f_{0}(980) \rightarrow \Upsilon(1 S) \eta \pi^{0}$

8. Reactions violating isotopic invariance in the central region

8.1. Reactions $p p \rightarrow p\left(f_{1}(1285) / f_{1}(1420)\right) p \rightarrow$ $p\left(\pi^{+} \pi^{-} \pi^{0}\right) p$;

8.2. Reaction $p p \rightarrow p\left(a_{0}^{0}(980)\right) p \rightarrow p\left(\eta \pi^{0}\right) p$

\section{Conclusion}

\section{References}

In memory of Dmitrii Vasil'evich Shirkov

\section{Introduction. Nature of scalar mesons}

In April 2016, one of the authors of this review (NNA) delivered a plenary report "37 Years with Light Scalar Mesons. The Lessons Learned" at the International conference on the Physics of Fundamental Interaction devoted to the 60th anniversary the Joint Institute for Nuclear Research in Dubna [1]. The main topic of the report 
was a threshold phenomenon discovered in experiments not long before the conference, which is now known as the mixing of $a_{0}^{0}(980)$ and $f_{0}(980)$ resonances. It was theoretically predicted in [2] as early as 1979 (see also [3, 4]). A special report on the problem related to strong violation of isotopic invariance in the production of light scalar mesons [5] was delivered at the 14th International Workshop on Tau-Lepton Physics held in Beijing in September 2016. An extended version of that report is the basis of this review.

The problem of light scalar mesons with masses $\lesssim 1$ $\mathrm{GeV}, \sigma(600), \kappa(800), a_{0}(980)$, and $f_{0}(980)$, has remained one of the most intriguing topics of hadron spectroscopy for several decades. The physics of light scalar mesons has been repeatedly discussed in Physics-Uspekhi [4, 68]. An impressive array of data on light scalars has been collected to date [9]. The nontrivial nature of these states is virtually uncontested. In particular, there is much evidence of their four-quark $q^{2} \bar{q}^{2}$ structure, and it is a matter for lively discussions. The number of publications devoted to light scalar mesons is truly immense. Some understanding of how theoretical and experimental explorations related to light scalar mesons have been developing can be gained, for example, from studies and reviews [1 30].

The search for light $\mathrm{s}$ and $\mathrm{k}$ mesons commenced as early as the 1960s, and at the same time preliminary information about these mesons appeared in Particle Data Group (PDG) reviews. The linear $\sigma$-model 31 33] was used as a theoretical tool to search for scalar mesons; this model takes spontaneous breaking of chiral symmetry into account and contains pseudoscalar mesons as Goldstone bosons. It has remarkably proved to be an effective lowenergy realization of quantum chromodynamics (QCD). Narrow scalar resonances, the isovector $a_{0}(980)$ and the isoscalar $f_{0}(980)$, were discovered in the late 1960 s and early 1970s.

In regard to $\sigma$ and $\kappa$ mesons, protracted unsuccessful attempts to confirm their existence in a conclusive way resulted in general disappointment, and information about those particles disappeared from the PDG reviews. The main argument against their existence was that the phase shifts in both $\pi \pi$ and $K \pi$ scattering fail to pass through $90^{\circ}$ at the assumed resonance masses. Nevertheless, experimental and theoretical studies of reactions in which the $\sigma$ and $\kappa$ states could manifest themselves continued.

The situation changed completely when the $S$-wave amplitude of the $\pi \pi$ scattering with the isospin $I=0$ in the linear $\sigma$-model was shown to contain a negative background phase 34 that conceals the $\sigma$ meson, as a result of which the $\pi \pi$ scattering phase fails to pass through $90^{\circ}$ at the assumed resonance mass. This observation clearly showed that the (background) screening of the lightest broad scalar mesons is a phenomenon inherent in chiral dynamics. This idea has been embraced to initiate a new wave of theoretical and experimental studies of $\sigma$ and $\kappa$ mesons. As a result, beginning in 1996, the light $\sigma$ resonance, and beginning in 2004, the light $\kappa$ resonance reappeared in particle-physics reviews [35, 36]. In study [37], which focused on the lightest scalar in the $S U(2)_{L} \times S U(2)_{R}$ linear $\sigma$-model, the existence of chiral screening of the $\sigma$ resonance was demonstrated in a joint description of low-energy data on the $\pi \pi \rightarrow \pi \pi$ and $\gamma \gamma \rightarrow \pi^{0} \pi^{0}$ reactions. It was discovered that the $\sigma(600) \rightarrow \gamma \gamma$ decay is a four-quark transition [8, 37].

Hadron scalar channels in the range $\lesssim 1 \mathrm{GeV}$ became a stumbling block for QCD, because neither the perturbation theory nor the sum rules for individual resonances are applicable in these channels. However, the nature of $\sigma(600), \kappa(800), a_{0}(980)$, and $f_{0}(980)$ light scalar mesons is the central point for understanding the chiral symmetry mechanism that emerges as a result of confinement, and hence for understanding confinement itself. Jaffe noted in 1977 that the quantum MIT bag model, which incorporates confinement in a phenomenological way, contains a nonet of light four-quark scalar states [13]. Jaffe suggested that $a_{0}(980)$ and $f_{0}(980)$ could be members of a nonet with the following symbolic quark structures: $\quad a_{0}^{+}(980)=u \bar{d} s \bar{s}, \quad a_{0}^{0}(980)=(u \bar{u}-d \bar{d}) s \bar{s} / \sqrt{2}$, $a_{0}^{-}(980)=d \bar{u} s \bar{s}$ and $f_{0}(980)=(u \bar{u}+d \bar{d}) s \bar{s} / \sqrt{2}$. From that time on, the $a_{0}^{0}(980)$ and $f_{0}(980)$ resonances have been the light-quark spectroscopy favorites.

Light scalar mesons have been explored in reactions of virtually all conceivable types using accelerators currently under operation. First, strong reactions in which pairs of pseudoscalar mesons $\pi^{+} \pi^{-}$, $K \bar{K}, \pi \eta, K \pi$, etc. are produced in $\pi N, K N$, and $N \bar{N}$ collisions have been studied (see, e.g., review [4]). It was shown in the late 1980s that studying the radiation decays $\phi(1020) \rightarrow \gamma a_{0}(980) \rightarrow \gamma \pi^{0} \eta$ and $\phi(1020) \rightarrow \gamma f_{0}(980) \rightarrow \gamma \pi \pi$ can shed light on the problem of $a_{0}(980)$ and $f_{0}(980)$ mesons [18]. This issue was under consideration from different perspectives for a decade, until the first experimental results appeared in 1988 [3844.

The decays $\phi(1020) \rightarrow \gamma a_{0}(980) \rightarrow \gamma \pi^{0} \eta \quad$ and $\phi(1020) \rightarrow \gamma f_{0}(980) \rightarrow \gamma \pi \pi$ have been studied to date not only theoretically but also experimentally with $e^{+} e^{-}$ colliders using the Spherical Neutral Detector (SND) 45 48 and the CMD-2 (Cryogenic Magnetic Detector) [49, 50] at the Novosibirsk- based Budker Institute of Nuclear Physics, Siberian Branch of the Russian Academy of Sciences, and the KLOE detector (K LOng Experiment) at the Frascati $\phi$-factory (Italy) 51 57].

The data from these experiments motivated theoretical studies that yielded the first arguments in favor of the four-quark nature of the $f_{0}(980)$ and $a_{0}(980)$ states [1, 7, 18, 41 44, 58 67]. It was shown in [41, 65, 66], for example, that light scalar mesons in $\phi(1020)$-meson radiative decays, $\phi(1020) \rightarrow \gamma f_{0}(980)$ and $\phi(1020) \rightarrow$ $\gamma a_{0}^{0}(980)$, are generated at small distances, implying that the light scalar mesons are compact states rather than loosely bound molecules. It was also shown in $60-62$ that radiative decays of the $\phi(1020)$ meson into light scalar mesons are four-quark transitions and, in the ap- 
proach based on a large number of colors $N_{c}$, correspond to the four-quark nature of light scalars.

Experimental studies of light scalar mesons in photonphoton collisions, more specifically in the reactions $\gamma \gamma \rightarrow \pi^{+} \pi^{-}, \gamma \gamma \rightarrow \pi^{0} \pi^{0}$ and $\gamma \gamma \rightarrow \pi^{0} \eta$, which commenced as early as the $1980 \mathrm{~s}$, are still in progress. It was predicted in 1982 [68, 69] that if the $a_{0}(980)$ and $f_{0}(980)$ mesons have a four-quark structure, the rates of their production in photon-photon collisions must be suppressed by an order of magnitude compared to the case of a twoquark structure. An estimate $\Gamma_{a_{0}^{0} \rightarrow \gamma \gamma} \approx \Gamma_{f_{0} \rightarrow \gamma \gamma} \approx 0.27$ $\mathrm{keV}$ was obtained in a four-quark model [68, 69], which was supported by experiment $[9,70-72$. The situation with $a_{0}(980)$ that emerged after the Crystal Ball collaboration [70] had for the first time measured the cross section of the reaction $\gamma \gamma \rightarrow a_{0}^{0}(980) \rightarrow \pi^{0} \eta$ was analyzed in detail in [73]. A dynamic model was proposed in that study for the amplitude of a two-photon decay of the $a_{0}(980)$ resonance (including the $K^{+} K^{-}$-loop mechanism of the transition $a_{0}(980) \rightarrow K^{+} K^{-} \rightarrow \gamma \gamma$ ), which agrees well with the data and demonstrates that the production of $a_{0}(980)$ in photon-photon collisions is suppressed.

Studies of light scalar mesons in photon-photon collisions at the B-factory in Japan [74 78], which opened the era of super-precise statistics, not only have confirmed predictions made 35 years earlier but also enabled reaching the conclusion [8, 79, 80] that the production mechanisms are four-quark transitions, thus signaling the fourquark nature of light scalar mesons.

It was shown in [81, 82] that data on semileptonic decays of heavy quarkonia are evidence of the four-quark nature of isoscalar $\sigma$ [or $\left.f_{0}(500)\right]$ and $f_{0}(980)$ mesons and provide a unique opportunity to explore the nature of light isovector scalar mesons in the nearest future.

The $f_{0}(980), a_{0}(980), \sigma(600)$, and $\kappa(800)$ resonances are now actively being studied in the numerous decays of heavy quarkonia, $J / \psi, D_{s}, D, B_{s}$, and $B$ by the LHCb (Large Hadron Collider beauty experiment) collaboration, BaBar, FOCUS, CLEO, Belle, BES III (Beijing Spectrometer III), etc. (see, e.g., reviews [9, 8386]). We note that the studies relating to the four-quark structure of light scalar mesons have paved the way for the 'bold' search for similar objects in the families of mesons that contain heavy $\mathrm{c}$ and $\mathrm{b}$ quarks. The majority of theoretical and experimental teams all over the world that explore elementary particle physics are now studying different four-quark states. One might expect that the Belle II detector on the SuperKEK B accelerator (Japan) will provide new data on production in photon-photon collisions of tensor four-quark states, including the tensor exotic four-quark state (E) with the isospin $I=2$ and mass in the vicinity of the rr threshold $[6,68,69,687,88]$. Along with the predicted suppression of the two-photon widths of $a_{0}(980)$ and $f_{0}(980)$ resonances, a spectacular manifestation of that exotic state in the reactions $\gamma \gamma \rightarrow \rho^{0} \rho^{0}$ and $\gamma \gamma \rightarrow \rho^{+} \rho^{-}$was predicted in 68, 69]. It was confirmed later in the experiments of three groups, JADE (Japan, Deutschland, Eng- land) [89], ARGUS (A Russian-German-United StatesSwedish collaboration) [90], and CELLO [91], which were performed at the PETRA accelerator (an abbreviation of Positron-Elektron Tandem Ring Anlage, the German name of the facility) at the DESY (Deutsches Elecktronen Synchrotron) particle physics research center (see, in relation to this, [6, 87, 88] ). Without a doubt, to conclusively prove the existence of a state with the quantum numbers $I^{G}\left(J^{P C}\right)=2^{+}\left(2^{++}\right)$and mass $\approx 1.5 \mathrm{GeV}$, it has to be discovered in charged $\rho^{ \pm} \rho^{ \pm}[6,92]$ and $\rho^{ \pm} \rho^{0}$ [6, 93] channels. Because the $E^{ \pm}$coupling to the $\gamma \rho^{ \pm}$ channel is strong, we believe that photoproduction reactions $\gamma N \rightarrow E^{ \pm} N \rightarrow \rho^{ \pm} \rho^{0} N$ and $\gamma N \rightarrow E^{ \pm} \Delta \rightarrow \rho^{ \pm} \rho^{0} \Delta$ (which occur owing to $\rho^{ \pm}$exchange) at the Jefferson Laboratory (USA) are the most promising ones for its search.

Each upgrade of accelerators and detectors triggers a new wave in studying light scalar mesons. The mechanisms of their production and decay and the shapes of corresponding mass distributions are studied at a new level of accuracy. The immense wealth of data with large statistics obtainable using modern-day accelerators will undoubtedly enable researchers to substantially improve the accuracy of scalar-resonance parameters and fruitfully continue the already commenced studies of fine threshold phenomena related to the violation of isotopic invariance in the region of $a_{0}(980)$ and $f_{0}(980)$ resonances in the $\pi^{+} \pi^{-}$and $\eta \pi^{0}$ mass spectra.

\section{The $a_{0}^{0}(980)-f_{0}(980)$ mixing as a threshold phenomenon. Amplitude and phase of the $a_{0}^{0}(980) \rightarrow\left(K^{+} K^{-}+\right.$ $\left.K^{0} \bar{K}^{0}\right) \rightarrow f_{0}(980)$ transition. First search proposals}

Experimental studies of effects related to the mixing of particles with different isotopic spins and close masses were always the focus of attention. The characteristic 'mass' (amplitude) squared of transitions such as $\rho^{0}-\omega$ and $\pi^{0}-\eta$ is approximately ${ }^{1} 0.003 \mathrm{GeV}^{2}$ or $\approx\left(m_{d}-m_{u}\right) \times 1 \mathrm{GeV}$. We here discuss the effects of isotopic symmetry breaking, the amplitude of which is $\sim \sqrt{m_{d}-m_{u}}$ (rather than $\sim\left(m_{d}-m_{u}\right)$, as usually occurs), and begin with the amplitude of transitions between $a_{0}^{0}(980)$ and $f_{0}(980)$ resonances.

The $a_{0}^{0}(980)$ and $f_{0}(980)$ resonances are located at the $K \bar{K}$ channel threshold and therefore experience its strong influence (see, e.g., [12, 14-16]). This essentially distinguishes the $a_{0}^{0}(980)-f_{0}(980)$ mixing from the wellstudied $\rho^{0}-\omega$ mixing. The point is that due to the vicinity of $a_{0}^{0}(980)$ and $f_{0}(980)$ resonances to the $K \bar{K}$ thresh-

\footnotetext{
1 This means that the order of magnitude of the amplitudes of those transitions is determined by the differences between the masses squared of the particles in isotopic meson multiplets. For example, the amplitude of the $\pi^{0}-\eta$ transition is $\left|\Pi_{\pi^{0} \eta}\right| \approx$ $m_{\pi}^{2}\left(m_{d}-m_{u}\right) /\left[\sqrt{3}\left(m_{d}+m_{u}\right)\right] \approx\left(m_{K^{0}}^{2}-m_{K^{+}}^{2}+m_{\pi^{+}}^{2}-m_{\pi^{0}}^{2}\right) / \sqrt{3}$ [94].
} 


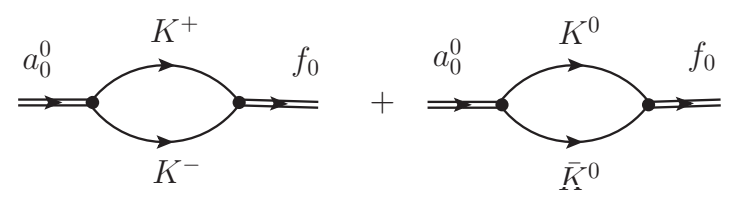

Figure 1: $K \bar{K}$-loop mechanism of the $a_{0}^{0}(980)-f_{0}(980)$ mixing.

old, the $a_{0}^{0}(980)-f_{0}(980)$ transition is significantly determined by contributions from the $K^{+} K^{-}$and $K^{0} \bar{K}^{0}$ intermediate states: $a_{0}^{0}(980) \rightarrow\left(K^{+} K^{-}+K^{0} \bar{K}^{0}\right) \rightarrow f_{0}(980)$ (Fig. 1). In the region between the $K^{+} K^{-}$and $K^{0} \bar{K}^{0}$ thresholds, whose width is $8 \mathrm{MeV}$, the amplitude of the $a_{0}^{0}(980)-f_{0}(980)$ transition $\Pi_{a_{0}^{0} f_{0}}(m)$ (here and below, $m$ is the invariant virtual mass of the scalar resonance) is $\sim \sqrt{m_{d}-m_{u}}$ rather than $\sim\left(m_{d}-m_{u}\right)$, as could be expected from general considerations. Outside this region, $\Pi_{a_{0}^{0} f_{0}}(m)$ rapidly decreases and tends to $\sim\left(m_{d}-m_{u}\right)$. Indeed, the sum of the diagrams shown in Fig. 1 converges and, given the isotopic symmetry of the coupling constants $g_{a_{0}^{0} K^{+} K^{-}}=-g_{a_{0}^{0} K^{0} \bar{K}^{0}}$ and $g_{f_{0} K^{+} K^{-}}=g_{f_{0} K^{0} \bar{K}^{0}}$, yields the following contribution to the amplitude of the $a_{0}^{0}(980)-f_{0}(980)$ transition:

$$
\begin{aligned}
& \Pi_{a_{0}^{0} f_{0}}(m)=\frac{g_{a_{0}^{0} K^{+} K^{-}} g_{f_{0} K^{+} K^{-}}}{16 \pi}\left[i \left(\rho_{K^{+} K^{-}}(m)\right.\right. \\
& \left.-\rho_{K^{0} \bar{K}^{0}}(m)\right)-\frac{\rho_{K^{+} K^{-}}(m)}{\pi} \ln \frac{1+\rho_{K^{+} K^{-}}(m)}{1-\rho_{K^{+} K^{-}}(m)} \\
& \left.+\frac{\rho_{K^{0} \bar{K}^{0}}(m)}{\pi} \ln \frac{1+\rho_{K^{0} \bar{K}^{0}}(m)}{1-\rho_{K^{0} \bar{K}^{0}}(m)}\right],
\end{aligned}
$$

where $\rho_{K \bar{K}}(m)=\sqrt{1-4 m_{K}^{2} / m^{2}}$ for $0 \leq m \leq 2 m_{K}$ and $\rho_{K \bar{K}}(m)$ must be replaced with $i\left|\rho_{K \bar{K}}(m)\right|$ for $0 \leq m \leq$ $2 m_{K}$. The resonance-like behavior of the absolute value and phase of amplitude (1) is visually displayed in Fig. 2. We represent $\Pi_{a_{0}^{0} f_{0}}(m)$ near the $K \bar{K}$ threshold as a series in $\rho_{K^{+} K^{-}}(m)$ and $\rho_{K^{0} \bar{K}^{0}}(m)$ :

$$
\begin{aligned}
& \Pi_{a_{0}^{0} f_{0}}(m)=\frac{g_{a_{0}^{0} K^{+} K^{-}} g_{f_{0} K^{+} K^{-}}}{16 \pi}\left[i \left(\rho_{K^{+} K^{-}}(m)\right.\right. \\
& \left.-\rho_{K^{0} \bar{K}^{0}}(m)\right)-\frac{2}{\pi}\left(\rho_{K^{+} K^{-}}^{2}(m)-\rho_{K^{0} \bar{K}^{0}}^{2}(m)\right) \\
& \left.+O\left(\rho_{K^{+} K^{-}}^{4}(m)-\rho_{K^{0} \bar{K}^{0}}^{4}(m)\right)+\ldots\right] \\
& =\frac{g_{a_{0}^{0} K^{+} K^{-}} g_{f_{0} K^{+} K^{-}}}{16 \pi}\left[i\left(\rho_{K^{+} K^{-}}(m)-\rho_{K^{0} \bar{K}^{0}}(m)\right)\right. \\
& +\frac{8\left(m_{K^{0}}^{2}-m_{K^{+}}^{2}\right)}{\pi m^{2}} \\
& \left.+O\left(\rho_{K^{+} K^{-}}^{4}(m)-\rho_{K^{0} \bar{K}^{0}}^{4}(m)\right)+\ldots\right] .
\end{aligned}
$$
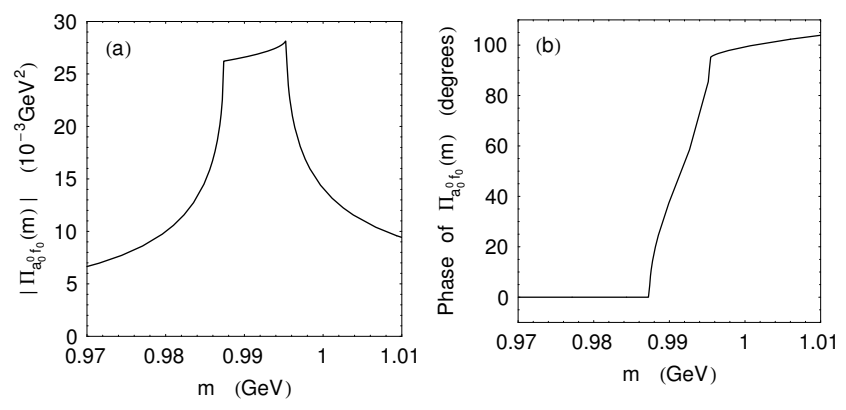

Figure 2: (a) Absolute value of the $a_{0}^{0}(980)-f_{0}(980)$ mixing amplitude: an example. (b) The phase of the $a_{0}^{0}(980)-$ $f_{0}(980)$ mixing changes by $90^{\circ}$ between the $K^{+} K^{-}$and $K^{0} \bar{K}^{0}$ thresholds, where the absolute value of the amplitude is practically constant and has the maximum value.

In the region between the $K^{+} K^{-}$and $K^{0} \bar{K}^{0}$ thresholds, the absolute value of the first term in (2) is

$$
\begin{aligned}
& \left|\Pi_{a_{0}^{0} f_{0}}(m)\right| \approx \frac{\left|g_{a_{0}^{0} K^{+} K^{-}} g_{f_{0} K^{+} K^{-}}\right|}{16 \pi} \sqrt{\frac{2\left(m_{K^{0}}-m_{K^{+}}\right)}{m_{K^{0}}}} \\
& \approx 0.127 \frac{\mid g_{a_{0} K^{+} K^{-} g_{f_{0} K^{+} K^{-}} \mid}^{16 \pi}}{2} \simeq 0.03 \mathrm{GeV}^{2} \\
& \approx m_{K} \sqrt{m_{K^{0}}^{2}-m_{K^{+}}^{2}} \approx m_{K}^{2} 0.127 \\
& \approx m_{K}^{3 / 2} \sqrt{2\left(m_{d}-m_{u}\right)} .
\end{aligned}
$$

In deriving this estimate for $\left|\Pi_{a_{0}^{0} f_{0}}\left(m \approx 2 m_{K}\right)\right|$ and plotting the $\left|\Pi_{a_{0}^{0} f_{0}}(m)\right|$ function in Fig. 2, we used the values of the $g_{a_{0}^{0} K^{+} K^{-}}$and $g_{f_{0} K^{+} K^{-}}$constants that were determined in [95] from the analysis of the first data of the BES III collaboration [96] on the $a_{0}^{0}(980)-f_{0}(980)$ mixing (see details in Section 4.3).

As was noted above, for the $\rho^{0}-\omega$ and $\pi^{0}-\eta$ transitions, $\left|\Pi_{\rho^{0} \omega}\right| \approx\left|\Pi_{\pi^{0} \eta}\right| \approx 0.003 \mathrm{GeV}^{2} \approx\left(m_{d}-m_{u}\right) \times 1$ $\mathrm{GeV}$.

We emphasize that the first term in the right-hand side of Eqn (2) is theoretically determined in an unambiguous way using the $a_{0}^{0}(980)$ and $f_{0}(980)$ coupling constants with the $K \bar{K}$ channel, as follows from the unitarity condition. Numerous data analyses indicate the four-quark nature of $f_{0}(980)$ and $a_{0}^{0}(980)$ resonances and their strong coupling with the superallowed decay into the $K \bar{K}$ channel [1, 17, 13]. The contribution from other intermediate states is, generally speaking, of the same order as the second term in (2), i.e., an order of magnitude smaller. Therefore, the $a_{0}^{0}(980)-f_{0}(980)$ transition is virtually completely driven by the first term in the righthand side of Eqn (2). As was noted above, the amplitude $\Pi_{a_{0}^{0} f_{0}}(m)$ rapidly decreases outside the region of the $K \bar{K}$ thresholds, and we can naturally expect the violation of isotopic invariance to be relatively small here, of the order of $\left(m_{K^{0}}-m_{K^{+}}\right) / m_{K^{0}} \approx 1 / 125$. Such contributions, which are incalculable in practice, can be ignored in the first approximation. 
Because the amplitude $\Pi_{a_{0}^{0} f_{0}}(m)$ is not small between the $K^{+} K^{-}$and $K^{0} \bar{K}^{0}$ thresholds, all orders of the $a_{0}^{0}(980)-f_{0}(980)$ mixing must be taken into account in the propagator $G_{a_{0}^{0} f_{0}}(m)$ that describes the $a_{0}^{0}(980) \rightarrow$ $f_{0}(980)$ and reverse transitions [2, 3],

$$
G_{a_{0}^{0} f_{0}}(m)=\frac{\Pi_{a_{0}^{0} f_{0}}(m)}{D_{a_{0}^{0}}(m) D_{f_{0}}(m)-\Pi_{a_{0}^{0} f_{0}}^{2}(m)},
$$

where $D_{r}(s)$ is the inverse propagator of the unmixed resonance $r$ with mass $m_{r}\left(r=a_{0}^{0}(980), f_{0}(980)\right)$,

$$
D_{r}(m)=m_{r}^{2}-m^{2}+\sum_{a b}\left[\operatorname{Re} \Pi_{r}^{a b}\left(m_{r}\right)-\Pi_{r}^{a b}(m)\right],
$$

$a b=\left(\eta \pi^{0}, K^{+} K^{-}, K^{0} \bar{K}^{0}, \eta^{\prime} \pi^{0}\right)$ for $r=a_{0}^{0}(980)$ and $a b=\left(\pi^{+} \pi^{-}, \pi^{0} \pi^{0}, K^{+} K^{-}, K^{0} \bar{K}^{0}, \eta \eta\right)$ for $r=f_{0}(980)$, $\Pi_{r}^{a b}(m)$ is the diagonal matrix element of the polarization operator of a resonance r, corresponding to the contribution of the $a b$ intermediate state. ${ }^{2}$

The propagators of $a_{0}^{0}(980)$ and $f_{0}(980)$ resonances, $1 / D_{a_{0}^{0}}(m)$ and $1 / D_{f_{0}}(m)$, built with consideration for the

2 The expressions for $\Pi_{r}^{a b}(m)$ in different $m$ regions are related to each other by analytic continuation [15, 95]. If $m>m_{a}+m_{b}$, then

$$
\begin{gathered}
\Pi_{r}^{a b}(m)=\frac{g_{r a b}^{2}}{16 \pi}\left[\frac{m_{a b}^{(+)} m_{a b}^{(-)}}{\pi m^{2}} \ln \frac{m_{b}}{m_{a}}\right. \\
\left.+\rho_{a b}(m)\left(i-\frac{1}{\pi} \ln \frac{\sqrt{m^{2}-m_{a b}^{(-) 2}}+\sqrt{m^{2}-m_{a b}^{(+) 2}}}{\sqrt{m^{2}-m_{a b}^{(-) 2}}-\sqrt{m^{2}-m_{a b}^{(+) 2}}}\right)\right],
\end{gathered}
$$

where $g_{r a b}$ is the constant of $r$ coupling to $a b, \rho_{a b}(m)=\left(m^{2}-\right.$ $\left.m_{a b}^{(+)}\right)^{1 / 2}\left(m^{2}-m_{a b}^{(-) 2}\right)^{1 / 2} / m^{2}, m_{a b}^{( \pm)}=m_{a} \pm m_{b}$, and $m_{a} \geq m_{b}$;

$$
\operatorname{Im} \Pi_{r}^{a b}(m)=m \Gamma_{r \rightarrow a b}(m)=\frac{g_{r a b}^{2}}{16 \pi} \rho_{a b}(m) .
$$

$$
\begin{aligned}
& \text { If } m_{a b}^{(-)}<m<m_{a b}^{(+)} \text {, then } \\
& \qquad \Pi_{r}^{a b}(m)=\frac{g_{r a b}^{2}}{16 \pi}\left[\frac{m_{a b}^{(+)} m_{a b}^{(-)}}{\pi m^{2}} \ln \frac{m_{b}}{m_{a}}\right. \\
& \left.-\rho_{a b}(m)\left(1-\frac{2}{\pi} \arctan \frac{\sqrt{m_{a b}^{(+) 2}-m^{2}}}{\sqrt{m^{2}-m_{a b}^{(-) 2}}}\right)\right],
\end{aligned}
$$

where $\rho_{a b}(m)=\left(m_{a b}^{(+) 2}-m^{2}\right)^{1 / 2}\left(m^{2}-m_{a b}^{(-) 2}\right)^{1 / 2} / m^{2}$. If $m \leq m_{a b}^{(-)}$, then

$$
\begin{gathered}
\Pi_{r}^{a b}(m)=\frac{g_{r a b}^{2}}{16 \pi}\left[\frac{m_{a b}^{(+)} m_{a b}^{(-)}}{\pi m^{2}} \ln \frac{m_{b}}{m_{a}}\right. \\
\left.+\rho_{a b}(m) \frac{1}{\pi} \ln \frac{\sqrt{m_{a b}^{(+) 2}-m^{2}}+\sqrt{m_{a b}^{(-) 2}-m^{2}}}{\sqrt{m_{a b}^{(+) 2}-m^{2}}-\sqrt{m_{a b}^{(-) 2}-m^{2}}}\right],
\end{gathered}
$$

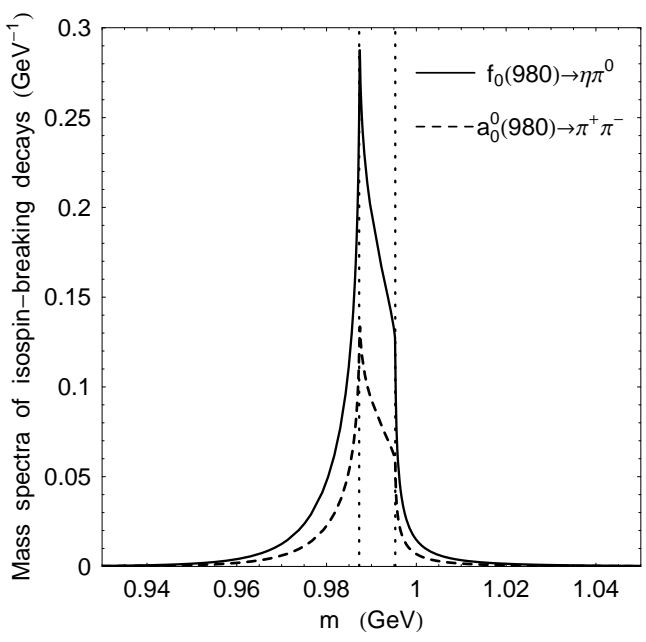

Figure 3: Mass spectra in isospin-violating decays $f_{0}(980) \rightarrow$ $\eta \pi^{0}$ and $a_{0}(980) \rightarrow \pi^{+} \pi^{-}$driven by the $a_{0}^{0}(980)-f_{0}(980)$ mixing. The solid and dashed curves are basically similar to each other. The dotted vertical lines show positions of the $K^{+} K^{-}$and $K^{0} \bar{K}^{0}$ thresholds.

finite width of the particles, satisfy the Källén-Lehmann representation in a broad range of the coupling constant $g_{r a b}$ and therefore ensure that the decay probabilities $\sum_{a b} B R(r \rightarrow a b)$ sum up to unity ( $B R$ is the branching ratio) [97].

We now present estimates for the probabilities of isospin-violating decays $f_{0}(980) \rightarrow \eta \pi^{0}$ and $a_{0}^{0}(980) \rightarrow$ $\pi^{+} \pi^{-}$that are due to the $a_{0}^{0}(980)-f_{0}(980)$ mixing. Using the constants of the coupling of $a_{0}^{0}(980)$ and $f_{0}(980)$ resonances to $\pi \pi, K \bar{K}, \eta \pi$, etc. channels [95], we obtain

$$
\begin{gathered}
B R\left(f_{0}(980) \rightarrow K \bar{K} \rightarrow a_{0}^{0}(980) \rightarrow \eta \pi^{0}\right) \\
=\int\left|G_{a_{0}^{0} f_{0}}(m)\right|^{2} \frac{2 m^{2} \Gamma_{a_{0}^{0} \rightarrow \eta \pi^{0}}(m)}{\pi} d m \approx 0.3 \%, \\
B R\left(a_{0}^{0}(980) \rightarrow K \bar{K} \rightarrow f_{0}(980) \rightarrow \pi^{+} \pi^{-}\right) \\
=\int\left|G_{a_{0}^{0} f_{0}}(m)\right|^{2} \frac{2 m^{2} \Gamma_{f_{0} \rightarrow \pi^{+} \pi^{-}}(m)}{\pi} d m \approx 0.14 \% .
\end{gathered}
$$

Figure 3 shows the mass spectra that correspond to integrands in Eqns (10) and (11). A characteristic feature of those decays is that the $\eta \pi^{0}$ and $\pi^{+} \pi^{-}$mass spectra are dominated by narrow resonance structures in the vicinity of the $K \bar{K}$ thresholds.

It is also of interest to estimate the cross section of the $G$-parity-forbidden $S$-wave process $\pi^{+} \pi^{-} \rightarrow \eta \pi^{0}$ (which is not directly observable) that can occur owing to the $a_{0}^{0}(980)-f_{0}(980)$ mixing mechanism:

$$
\begin{gathered}
\sigma\left(\pi^{+} \pi^{-} \rightarrow \eta \pi^{0}\right)=\frac{16 \pi}{\rho_{\pi^{+} \pi^{-}}^{2}(m)} \\
\times \Gamma_{f_{0} \rightarrow \pi^{+} \pi^{-}}(m) \Gamma_{a_{0}^{0} \rightarrow \eta \pi^{0}}(m)\left|G_{a_{0}^{0} f_{0}}(m)\right|^{2} .
\end{gathered}
$$


The resonance-like behavior of $\sigma\left(\pi^{+} \pi^{-} \rightarrow \eta \pi^{0}\right)$ as a function of energy is virtually undistinguishable from that of the $\eta \pi^{0}$ mass spectrum (see the solid curve in Fig. $3)$. The estimated average value of $\sigma\left(\pi^{+} \pi^{-} \rightarrow \eta \pi^{0}\right)$ in the range between the $K^{+} K^{-}$and $K^{0} \bar{K}^{0}$ thresholds is $\approx 0.39 \mathrm{mb}$. This value is too large for a $G$-parity-forbidden process. The quoted value is about $8 \%$ of the unitary limit for the cross section of the allowed $S$-wave process $\pi^{+} \pi^{-} \rightarrow \pi^{0} \pi^{0}$, which is $32 \pi /\left[3 m \rho_{\pi^{+} \pi^{-}}(m)\right]^{2} \approx 4.8 \mathrm{mb}$ at $m \approx 2 m_{K}$.

If $a_{0}^{0}(980)$ and $f_{0}(980)$ resonances can be produced concurrently in a reaction (for example, in $\pi^{-} p$ collisions), their contributions mutually interfere in $\eta \pi^{0}$ and $\pi^{+} \pi^{-}$ mass spectra owing to the $a_{0}^{0}(980)-f_{0}(980)$ mixing. The corresponding interference effects are determined by the $a_{0}^{0}(980)-f_{0}(980)$ mixing amplitude and relative values of the initial $a_{0}^{0}(980)$ and $f_{0}(980)$ production amplitudes. The possibility of detecting the $a_{0}^{0}(980)-f_{0}(980)$ mixing is then significantly dependent on the choice of the particular reaction.

If selection rules allow production in a reaction of the $a_{0}^{0}(980)$ or $f_{0}(980)$ resonance alone, that reaction provides, in principle, an opportunity to directly measure $B R\left(a_{0}^{0}(980) \rightarrow \pi^{+} \pi^{-}\right)$or $B R\left(f_{0}(980) \rightarrow \eta \pi^{0}\right)$ that are due to the $a_{0}^{0}(980)-f_{0}(980)$ mixing.

We discuss various examples of reactions of both types in this review.

2.1. Peripheral reactions $\pi^{ \pm} N \rightarrow\left(a_{0}^{0}(980)\right.$, $\left.f_{0}(980)\right)(N, \Delta) \rightarrow \eta \pi^{0}(N, \Delta)$

We begin with high-energy reactions

$$
\pi^{ \pm} N \rightarrow\left(a_{0}^{0}(980), f_{0}(980)\right) N \rightarrow \eta \pi^{0} N,
$$

where one-pion exchange in the $\mathrm{t}$ channel becomes possible owing to the $a_{0}^{0}(980)-f_{0}(980)$ mixing, $\pi^{ \pm} N \rightarrow$ $f_{0}(980) N \rightarrow \eta \pi^{0} N[2,3,38]$. The one-pion exchange amplitudes are known to be large. Therefore, the effect of the $G$-parity-forbidden amplitude $\pi^{ \pm} N \rightarrow f_{0}(980) N \rightarrow$ $\eta \pi^{0} N$ is enhanced on the background of the allowed process $\pi^{ \pm} N \rightarrow a_{0}^{0}(980) N \rightarrow \eta \pi^{0} N$ at $|t| \lesssim 0.1 \mathrm{GeV}^{2}$ (where $t$ is the momentum squared transferred from the $\pi^{ \pm}$system to the $\eta \pi^{0}$ system). The differential cross section of reaction (13) can be represented as

$$
\frac{d \sigma}{d t d m}=\left| \pm M_{+-}^{a_{0}^{0}}\left(b_{1}\right)+M_{+-}^{f_{0}}(\pi)\right|^{2}+\left| \pm M_{++}^{a_{0}^{0}}\left(\rho_{2}\right)\right|^{2} .
$$

where $M_{+-}^{r}$ and $M_{++}^{r}$ are the $s$-channel helicity amplitudes with and without the nucleon helicity flip, which describe production of the resonance $r$ and its subsequent decay into $\eta \pi^{0}$. The symbols in parentheses indicate the type of Regge exchange for the given helicity amplitude (for this, we use symbols of the lightest particles lying on the Regge trajectories with the corresponding quantum numbers); the \pm sign means that the amplitudes of $a_{0}^{0}(980)$ production have different signs in the reactions $\pi^{-} p \rightarrow \eta \pi^{0} n$ and $\pi^{+} n \rightarrow \eta \pi^{0} p$. We recall that the $\rho_{2}$ state corresponds in today's notations to the quantum numbers $I^{G}\left(J^{P C}\right)=1^{+}\left(2^{--}\right)$; however, this state has not been identified yet as a peak in corresponding multiparticle mass spectra [9].

Regge amplitudes with $\rho_{2}$-exchange quantum numbers in the $\mathrm{t}$ channel (earlier referred to as the $Z$ exchange 99]) are nevertheless needed to describe data on differential cross sections of the reactions $\pi^{-} p \rightarrow a_{0}^{0}(980) n$, $\pi^{-} p \rightarrow \omega n, \pi N \rightarrow a_{2}(1320) N$, etc. These amplitudes can also be due to Regge cuts. The properties of the Regge amplitude $M_{++}^{a_{0}^{0}}\left(\rho_{2}\right)$ for the reaction $\pi^{-} p \rightarrow a_{0}^{0}(980) n$ have been considered in detail in [98].

The reaction $\pi^{-} p \rightarrow \eta \pi^{0} n$ has been studied at the Brookhaven National Laboratory at the laboratorysystem momentum of the incident pion $P_{l a b}^{\pi^{-}}=18.3$ $\mathrm{GeV} / c$ [100, 101], at the Protvino-based Institute of High Energy Physics at 32 and $38 \mathrm{GeV} / c$ 102, 103], and at CERN at $100 \mathrm{GeV} / c$ [103]. The contribution to the cross section of the $\pi^{-} p \rightarrow \eta \pi^{0} n$ process from the reggeized one-pion exchange (OPE) has the form [98]

$$
\begin{gathered}
\frac{d \sigma^{(\mathrm{OPE})}}{d t d m}=\frac{1}{\pi s^{2}} \frac{g_{\pi N N}^{2}}{4 \pi}\left[\frac{-t e^{\Lambda_{\pi}(s)\left(t-m_{\pi}^{2}\right)}}{\left(t-m_{\pi}^{2}\right)^{2}}\right] \\
\times m^{3} \rho_{\pi^{+} \pi^{-}}(m) \sigma\left(\pi^{+} \pi^{-} \rightarrow \eta \pi^{0}\right),
\end{gathered}
$$

where $s$ is the energy squared in the $\pi^{-} p$ center-of-mass system, $g_{\pi N N}^{2} /(4 \pi) \approx 14.6, \Lambda_{\pi}(s) / 2$ is the slope of the Regge $\pi$-pole residue, and the $\sigma\left(\pi^{+} \pi^{-} \rightarrow \eta \pi^{0}\right)$ cross section is given by Eqn (12) [see also the discussion that follows Eqn (12)]. According to a very conservative estimate quoted in [104, 105], at $P_{l a b}^{\pi^{-}}=18.3 \mathrm{GeV} / c$, the value of $d \sigma^{(\mathrm{OPE})} / d t$ at the maximum located at $t \approx-0.015 \mathrm{GeV}^{2}$ is $\approx 140 \mathrm{nb} / \mathrm{GeV}^{2}$, and the total cross section of the onepion exchange is $\sigma^{(\mathrm{OPE})} \approx 11 \mathrm{nb}$. These values respectively make about $15 \%$ of $\left[d \sigma / d t\left(\pi^{-} p \rightarrow a_{0}^{0}(980) n \rightarrow\right.\right.$ $\left.\left.\eta \pi^{0} n\right)\right]\left.\right|_{t \approx 0} \approx 940 \mathrm{nb} / \mathrm{GeV}^{2}$ and about $5.5 \%$ of the total cross section $\sigma\left(\pi^{-} p \rightarrow a_{0}^{0}(980) n \rightarrow \eta \pi^{0} n\right) \approx 200 \mathrm{nb}$ at $18.3 \mathrm{GeV} / c$ (see details in [98, 104, 105]). The BNL data on the reaction $\pi^{-} p \rightarrow a_{0}^{0}(980) n \rightarrow \eta \pi^{0} n$ were normalized in [98] (see also [104, 105], where the agreement of that normalization with the estimated cross section presented in [102] was noted).

Thus, $G$-parity-violating one-pion exchange can play an important role in forming the forward peak in the $d \sigma / d t\left(\pi^{-} p \rightarrow a_{0}^{0}(980) n \rightarrow \eta \pi^{0} n\right)$ cross section and influence the shape of the $\eta \pi^{0}$ mass spectrum in the region of the $a_{0}^{0}(980)$ resonance. Unfortunately, due to insufficient resolution in the invariant mass of the $\eta \pi^{0}$ system and variable $t$ and limited statistics (errors in cross sections), it is not possible to separate the signal from the $a_{0}^{0}(980)-f_{0}(980)$ mixing in the data of the BNL [100, 101] and IHEP [102, 103] experiments. More precise measurements of the $\pi^{-} p \rightarrow a_{0}^{0}(980) n \rightarrow \eta \pi^{0} n$ reaction remain a promising and interesting option in what regards detection of the $a_{0}^{0}(980)-f_{0}(980)$ mixing.

We note that the interference of the contributions from the $\pi$ and $b_{1}$ exchanges that occurs in the amplitude $M_{+-}$ [see (14)] was discussed in detail in [3] (see also subsequent studies [98, 104, 105]). Study [3] also contained 
estimates of possible manifestations of $a_{0}^{0}(980)-f_{0}(980)$ mixing in the reactions $\pi^{ \pm} N \rightarrow\left(a_{0}^{0}(980), f_{0}(980)\right) \Delta \rightarrow$ $\eta \pi^{0} \Delta$, where the interference of the $\pi$ and $b_{1}$ exchanges at small $t$ can be more significant than in $\pi^{ \pm} N \rightarrow$ $\left(a_{0}^{0}(980), f_{0}(980)\right) N \rightarrow \eta \pi^{0} N$.

2.2. Reactions $\left(K^{-}, \bar{K}^{0}\right) N \rightarrow\left(f_{0}(980), a_{0}^{0}(980)\right)$ $(\Lambda, \Sigma, \Sigma(1385)) \rightarrow\left(\pi^{+} \pi^{-} / \eta \pi^{0}\right)(\Lambda, \Sigma, \Sigma(1385))$

A somewhat different picture of $a_{0}^{0}(980)-f_{0}(980)$ interference can be expected at high energies in the reactions

$$
\begin{aligned}
\left(K^{-}, \bar{K}^{0}\right) N \rightarrow & \left(f_{0}(980), a_{0}^{0}(980)\right)(\Lambda, \Sigma, \Sigma(1385)) \\
& \rightarrow\left\{\begin{array}{l}
\left(\pi^{+} \pi^{-}\right)(\Lambda, \Sigma, \Sigma(1385)) \\
\left(\eta \pi^{0}\right)(\Lambda, \Sigma, \Sigma(1385))
\end{array}\right.
\end{aligned}
$$

The allowed $t$-channel exchanges are in this case the same as for the production of $a_{0}^{0}(980)$ and $f_{0}(980)$ resonances. If the Regge $K$-pole exchange dominates (exchanges with natural parity are forbidden), the interference phenomena in the $\pi^{+} \pi^{-}$and $\eta \pi^{0}$ mass spectra are calculated in an unambiguous way [3]:

$$
\begin{aligned}
& \frac{d N_{\pi^{+} \pi^{-}}}{d m}=C g_{f_{0} K^{+} K^{-}}^{2} \frac{2 m^{2} \Gamma_{f_{0} \rightarrow \pi^{+} \pi^{-}}(m)}{\pi\left|D_{f_{0}}(m)\right|^{2}} \\
& \times\left|1 \pm \frac{g_{a_{0}^{0} K^{+} K^{-}}}{g_{f_{0} K^{+} K^{-}}} G_{a_{0}^{0} f_{0}}(m) D_{f_{0}}(m)\right|^{2} \\
& \frac{d N_{\eta \pi^{0}}}{d m}=C g_{a_{0}^{0} K^{+} K^{-}}^{2} \frac{2 m^{2} \Gamma_{a_{0}^{0} \rightarrow \eta \pi^{0}}(m)}{\pi\left|D_{a_{0}^{0}}(m)\right|^{2}} \\
& \quad \times\left|1 \pm \frac{g_{f_{0} K^{+} K^{-}}}{g_{a_{0}^{0} K^{+} K^{-}}} G_{a_{0}^{0} f_{0}}(m) D_{a_{0}^{0}}(m)\right|^{2},
\end{aligned}
$$

The + and - signs in Eqns (17) and (18) correspond to reactions (16) with $K^{-}$and $\bar{K}^{0}$ mesons. It is generally assumed that the Regge residues are proportional to the coupling constants of the scalar resonances and $K^{+} K^{-}$. As a result of the interference, the resonance peaks in the reactions caused by $K^{-}$and $\bar{K}^{0}$ mesons are separated by 10 to $15 \mathrm{MeV}$ and have different shapes [3].

2.3. Reactions of $\overline{\boldsymbol{p}} \boldsymbol{n}$ annihilation at rest $\overline{\boldsymbol{p}} \boldsymbol{n} \rightarrow$ $\left(\pi^{-}, \rho^{-}\right) f_{0}(980) \rightarrow\left(\pi^{-}, \rho^{-}\right) \eta \pi^{0}$

In the reactions of $\bar{p} n$ annihilation at rest,

$$
\begin{gathered}
\bar{p} n \rightarrow \pi^{-} f_{0}(980) \rightarrow \pi^{-} \eta \pi^{0}, \\
\bar{p} n \rightarrow \rho^{-} f_{0}(980) \rightarrow \rho^{-} \eta \pi^{0},
\end{gathered}
$$

which occur owing to the $a_{0}^{0}(980)-f_{0}(980)$ mixing, a narrow peak is supposed to be observed in the $\eta \pi^{0}$ mass spectrum at $m \approx 2 m_{K}$, similar to that shown in Fig. 3. The $\bar{p} n$ annihilation at rest occurs in reaction (19) in the state with the $\pi^{-}$-meson quantum numbers, and in reaction $(20)$ with the $\rho^{-}$-meson quantum numbers. Therefore, production of the $\eta \pi^{0}$ system via the $a_{0}^{0}(980)$ resonance is forbidden in these reactions due to $G$-parity.
Thus, reactions (19) and (20) provide an opportunity to directly measure the value of $B R\left(f_{0}(980) \rightarrow \eta \pi^{0}\right)[3]$.

2.4. Decay $f_{1}(1285) \rightarrow a_{0}^{0}(980) \pi^{0} \rightarrow 3 \pi$

As was noted in [2], the $a_{0}^{0}(980)-f_{0}(980)$ mixing enables the $f_{1}(1285)$ meson with the quantum numbers $I^{G}\left(J^{P C}\right)=0^{+}\left(1^{++}\right)$to decay into $3 \pi$ :

$$
f_{1}(1285) \rightarrow a_{0}^{0}(980) \pi^{0} \rightarrow f_{0}(980) \pi^{0} \rightarrow 3 \pi .
$$

The decay $f_{1}(1285) \rightarrow a_{0}(980) \pi \rightarrow \eta \pi \pi$ is one of the main decay channels for the $f_{1}(1285)$ meson [9]. According to the estimate given in [3] ,

$$
\frac{\Gamma_{f_{1}(1285) \rightarrow a_{0}^{0} \pi^{0} \rightarrow \pi^{+} \pi^{-} \pi^{0}}}{\Gamma_{f_{1}(1285) \rightarrow a_{0}^{ \pm}(980) \pi^{\mp} \rightarrow \eta \pi^{+} \pi^{-}}} \approx 1-3 \%
$$

depending on the parameters of $f_{0}(980)$ and $a_{0}^{0}(980)$ resonances, which were varied in a rather broad range. This result initiated studies of the isospin-violating decay $f_{1}(1285) \rightarrow f_{0}(980) \pi^{0} \rightarrow \pi^{+} \pi^{-} \pi^{0}$. Resumed later (after more than 25 years), these studies yielded a rather interesting result. The modern-day situation with the $f_{1}(1285) \rightarrow 3 \pi$ decay is described in Sections 4 and 5 .

In concluding this section, we note that the interest in the phenomenon of $a_{0}^{0}(980)-f_{0}(980)$ mixing started gradually increasing beginning in 1995. For example, the $a_{0}^{0}(980)-f_{0}(980)$ mixing and the ways to observe it in experiment were repeatedly discussed in the decade that followed [20, 98, 100, 104 117].

A conceptually new proposal to search for $a_{0}^{0}(980)-$ $f_{0}(980)$ mixing was made in 2004. We proposed in 104, 105] to conduct an experiment using the $\pi^{-} p \rightarrow$ $\eta \pi^{0} n$ reaction on a polarized target, which allowed uniquely identifying the $a_{0}^{0}(980)-f_{0}(980)$ by a large and abrupt increase in the azimuthal asymmetry in the $\eta \pi^{0}$ production cross section in an $S$-wave with the $\eta \pi^{0}$ invariant mass varying in the region of the $K \bar{K}$ thresholds. The behavior of this asymmetry is primarily driven by the $90^{\circ}$ change in the amplitude of the $a_{0}^{0}(980)-f_{0}(980)$ transition between the $K \bar{K}$ thresholds. This proposal is discussed in Section 3.

\section{The $a_{0}^{0}(980)-f_{0}(980)$ mixing in polarization phenomena. Reaction $\pi^{-} p \uparrow \rightarrow \eta \pi^{0} n$}

The surge in the phase of the $a_{0}^{0}(980)-f_{0}(980)$ transition amplitudes shown in Fig. 2b has prompted an exploration of $a_{0}^{0}(980)-f_{0}(980)$ mixing in polarization phenomena [104, 105]. If the amplitude of the process with this spin configuration is dominated by the contribution due to the $a_{0}^{0}(980)-f_{0}(980)$ mixing, the spin asymmetry of the cross section must exhibit a surge near the $K \bar{K}$ thresholds. An example of this phenomenon is the $\pi^{-} p \uparrow \rightarrow\left(a_{0}^{0}(980), f_{0}(980)\right) n \rightarrow a_{0}^{0}(980) n \rightarrow \eta \pi^{0} n$ reaction on a polarized target. The corresponding differential cross section is

$$
\frac{d \sigma}{d t d m d \psi}=\frac{1}{2 \pi}\left[\frac{d \sigma}{d t d m}+I(t, m) P \cos \psi\right]
$$




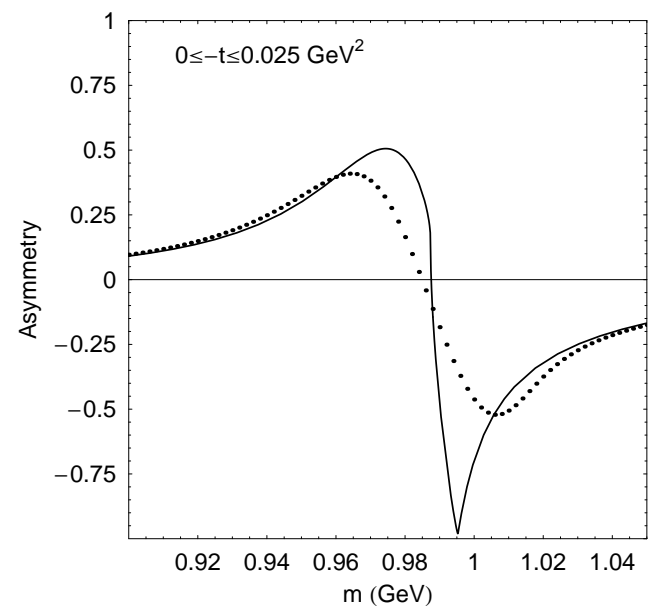

Figure 4: Manifestation of $a_{0}^{0}(980)-f_{0}(980)$ mixing in the reaction $\pi^{-} p \uparrow \rightarrow \eta \pi^{0} n$ on a polarized target at $P_{l a b}^{\pi^{-}}=18.3$ $\mathrm{GeV} / c$ in the model of $\rho_{2}$ and $\pi$ Regge exchanges [104, 105]. The solid curve shows the spin asymmetry $A(0 \leq-t \leq$ $\left.0.025 \mathrm{GeV}^{2}, m\right)$ as a function of the invariant mass of the $\eta \overline{\pi^{0}}$ system $\mathrm{m}$ (the dotted curve is the result of smoothing in $\mathrm{m}$ using a Gauss distribution with a dispersion of $10 \mathrm{MeV}$ ).

where $\psi$ is the angle between the normal to the reaction plane determined by the momenta of the incident $\pi^{-}$ meson and the final $\eta \pi^{0}$ system and the direction of the polarization of target protons perpendicular to the $\pi^{-}$beam axis, $P$ is the degree of that polarization, $I(t, m)=$ $2 \operatorname{Im}\left(M_{++} M_{+-}^{*}\right)$ describes the interference contribution that yields the azimuthal asymmetry of the cross section, and $d \sigma / d t d m=\left|M_{+-}\right|^{2}+\left|M_{++}\right|^{2}$ is the cross section on an unpolarized target. The values of $I(t, m)$ and $d \sigma / d t d m$ are used to determine the dimensionless normalized spin asymmetry $A(t, m)=I(t, m) /\left[d^{2} \sigma / d t d m\right]$, $-1 \leq A(t, m) \leq 1$.

Figure 4 illustrates the surge in the asymmetry as a function of $m$ in the region of the $K \bar{K}$ thresholds at $0 \leq-t \leq 0.025 \mathrm{GeV}^{2}$. It emerges as a result of interference between the isospin-conserving amplitude $M_{++}=M_{++}^{a_{0}^{0}}\left(\rho_{2}\right)$ and the amplitude $M_{+-}=M_{+-}^{f_{0}}(\pi)$ due to the $a_{0}^{0}(980)-f_{0}(980)$ mixing in the model of $\rho_{2}$ and $\pi$ Regge exchanges [see (14)]. This selection of the Regge mechanism model is not fortuitous: a description of the BNL 100] and IHEP 103 data for the differential cross section $d \sigma / d t\left(\pi^{-} p \rightarrow a_{0}^{0}(980) n \rightarrow \eta \pi^{0} n\right)$ on an unpolarized target does not require introducing $M_{+-}^{a_{0}^{0}}\left(b_{1}\right)$, an amplitude with the quantum numbers of the $b_{1}$ Regge exchange [see (14)]. Those data are perfectly well approximated in the $0 \leq-t \leq 0.6 \mathrm{GeV}^{2}$ range using a simple exponential function $\bar{d} \sigma / d t=C \exp (\Lambda t)$, i.e., they can be described using the amplitude $M_{++}^{a_{0}^{0}}\left(\rho_{2}\right)$, which does not vanish as $t \rightarrow 0$ (see, e.g., the solid curve in Fig. 5), while the contribution of the spin-flip amplitude is $M_{+-}^{a_{0}^{0}}\left(b_{1}\right) \sim \sqrt{-t}$. The value of the polarization

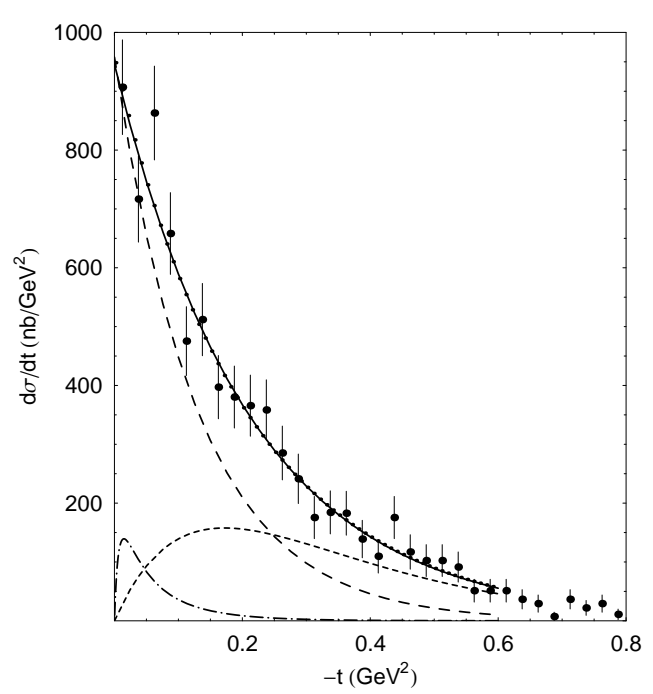

Figure 5: Dots with error bars are the normalized BNL data [100] for the differential cross section $d \sigma / d t$ of the reaction $\pi^{-} p \rightarrow a_{0}^{0}(980) n \rightarrow \eta \pi^{0} n$ at $P_{l a b}^{\pi^{-}}=18.3 \mathrm{GeV} / c$ [98]. The solid curve is the result of data approximation in the model of the $\rho_{2}$ Regge exchange. The dotted curve that virtually coincides with the solid one is an example of data approximation in the model of $\rho_{2}$ and $b_{1}$ Regge exchanges, whose contributions to $d \sigma / d t$ are respectively presented by the long-dashed and short-dashed curves. The dot-dashed curve shows the cross section $d \sigma^{(\mathrm{OPE})} / d t$ of the reaction $\pi^{-} p \rightarrow f_{0}(980) n \rightarrow a_{0}^{0}(980) n \rightarrow \eta \pi^{0} n$ at $P_{l a b}^{\pi^{-}}=18.3 \mathrm{GeV} / c$ (see details in [105]).

effects in the model of $\rho_{2}$ and $\pi$ Regge exchanges does not change much within the intervals $0 \leq-t \leq 0.05$, 0.1 , and $0.2 \mathrm{GeV}^{2}$. All the details of versions that occur when the contribution from the b1 exchange is taken into account (a version of this kind is illustrated in Fig. 5) can be found in [104, 105]. The main conclusion that can be drawn from the analysis made in the papers cited above is that the spin asymmetry emerging in any interval $0 \leq-t \leq 0.025, \ldots, 0.100 \mathrm{GeV}^{2}$ is in any event predicted to exhibit, due to an admixture of the $\pi$ exchange, an increase by a value close to unity in the $m$ range from 0.965 to $1.010 \mathrm{GeV}$.

It is noteworthy that detecting the spin asymmetry surge does not require high resolution in the invariant mass of the $\eta \pi^{0}$ system (see Fig. 4), which is of importance for identifying manifestations of the $a_{0}^{0}(980)-$ $f_{0}(980)$ mixing in the $\eta \pi^{0}$ mass spectrum in an unpolarized experiment. Moreover, it is expected that the energy dependence of the polarization effect is weak. Therefore, it can be explored in a broad $P_{l a b}^{\pi^{-}}$range, for example, from 8 to $100 \mathrm{GeV} / c$.

The reactions with polarized targets are still waiting 
to be explored experimentally.

\section{Detection of $a_{0}^{0}(980)-f_{0}(980)$ mixing}

Interest in the isospin-violating $a_{0}^{0}(980)-f_{0}(980)$ mixing has not abated over the last decade [5, 95, 96, 118 143]. Effects related to this phenomenon have been discovered and experimentally studied using the VES (Vertex Spectrometer) detector in Protvino 119 122 and the BES III detector in Beijing [96, 125, 134] in the reactions

$$
\begin{aligned}
& \pi^{-} N \rightarrow \pi^{-} f_{1}(1285) N \rightarrow \pi^{-} f_{0}(980) \pi^{0} N \\
& \rightarrow \pi^{-} \pi^{+} \pi^{-} \pi^{0} N \text { [119 122], } \\
& J / \psi \rightarrow \phi f_{0}(980) \rightarrow \phi a_{0}(980) \rightarrow \phi \eta \pi^{0} \text { [96], } \\
& \chi_{c 1} \rightarrow a_{0}(980) \pi^{0} \rightarrow f_{0}(980) \pi^{0} \rightarrow \pi^{+} \pi^{-} \pi^{0} \text { [96], (26) } \\
& J / \psi \rightarrow \gamma \eta(1405) \rightarrow \gamma f_{0}(980) \pi^{0} \rightarrow \gamma 3 \pi \text { [125], } \\
& J / \psi \rightarrow \phi f_{1}(1285) \rightarrow \phi f_{0}(980) \pi^{0} \rightarrow \phi 3 \pi \text { [134]. }
\end{aligned}
$$

The results of these experiments have shown quite clearly [5, 95, 135] that similar isospin violation effects, which are due to the mass difference of the $K^{+}$and $K^{0}$ mesons, can emerge not only as a result of the $a_{0}^{0}(980)-f_{0}(980)$ mixing alone but also from any mechanism of $K \bar{K}$-pair production with a definite isospin in the $S$-wave:

$$
\begin{aligned}
& X_{I=0} \rightarrow\left(K^{+} K^{-}+K^{0} \bar{K}^{0}\right) \rightarrow a_{0}^{0}(980) \rightarrow \eta \pi^{0}, \\
& X_{I=1} \rightarrow\left(K^{+} K^{-}+K^{0} \bar{K}^{0}\right) \rightarrow f_{0}(980) \rightarrow \pi^{+} \pi^{-} .
\end{aligned}
$$

Each mechanism of this kind generates both a narrow resonance peak in the $\eta \pi^{0}$ or $\pi^{+} \pi^{-}$mass spectrum (see Fig. 3 as an example) and a surge in the phase amplitude between the $K^{+} K^{-}$and $K^{0} \bar{K}^{0}$ thresholds (Fig. 2b). This implies that a new tool for studying the production mechanism and nature of light scalars is now available.

We discuss the effects discovered in reactions (24)-(28) in Sections 4.1-4.3 and 5.

4.1. VES experiment in Protvino: the reaction $\pi^{-} N \rightarrow \pi^{-} f_{1}(1285) N \rightarrow \pi^{-} f_{0}(980) \pi^{0} N \rightarrow$ $\pi^{-} \pi^{+} \pi^{-} \pi^{0} N$

The first preliminary observations of the isotopicsymmetry-violating decay $f_{1}(1285) \rightarrow \pi^{+} \pi^{-} \pi^{0}$ obtained using the VES detector at the Protvino accelerator [119] were reported in 2007 at the 12th International Conference on Hadron Spectroscopy (Hadron 2007) (see also $[120,121])$. The reaction of the diffraction production of the $\pi^{-} f_{1}(1285)$ system, $\pi^{-} N \rightarrow \pi^{-} f_{1}(1285) N$, was used as a source of $f_{1}(1285)$ mesons. This reaction was studied in $\pi^{-}$Be collisions at $P_{l a b}^{\pi^{-}}=27,37$, and $41 \mathrm{GeV} / c$. The signal from the $f_{1}(1285)$ resonance was sought in the $\pi^{+} \pi^{-} \pi^{0}$ decay channel and in the 'reference' $\eta \pi^{+} \pi^{-}$channel. It was found that a distinguishing feature of the $f_{1}(1285) \rightarrow \pi^{+} \pi^{-} \pi^{0}$ decay is the presence in the $\pi^{+} \pi^{-}$mass spectrum of a resonance-type structure in the vicinity of $f_{0}(980)$, which is characteristic of the isospin-violating $a_{0}^{0}(980)-f_{0}(980)$ mixing, $f_{1}(1285) \rightarrow a_{0}^{0}(980) \pi^{0} \rightarrow f_{0}(980) \pi^{0} \rightarrow \pi^{+} \pi^{-} \pi^{0}$ (see Section 2.4).
The final results of the VES experiment reported in 122] can be formulated as follows. The $f_{1}(1285) \rightarrow$ $\pi^{+} \pi^{-} \pi^{0}$ decay, which violates isotopic symmetry, has been discovered. Its branching ratio

$$
\begin{gathered}
\frac{B R\left(f_{1}(1285) \rightarrow f_{0}(980) \pi^{0} \rightarrow \pi^{+} \pi^{-} \pi^{0}\right)}{B R\left(f_{1}(1285) \rightarrow \eta \pi^{+} \pi^{-}\right)} \\
=(0.86 \pm 0.16 \pm 0.20) \%
\end{gathered}
$$

and the probability of the $f_{1}(1285) \rightarrow \pi^{+} \pi^{-} \pi^{0}$ decay have been measured:

$$
\begin{gathered}
B R\left(f_{1}(1285) \rightarrow f_{0}(980) \pi^{0} \rightarrow \pi^{+} \pi^{-} \pi^{0}\right) \\
\quad=(0.30 \pm 0.055 \pm 0.074) \% .
\end{gathered}
$$

The PDG data [9] on $B R\left(f_{1}(1285) \rightarrow a_{0}^{0}(980) \pi^{0} \rightarrow\right.$ $\left.\eta \pi^{0} \pi^{0}\right)$ yield

$$
\begin{gathered}
\frac{B R\left(f_{1}(1285) \rightarrow f_{0}(980) \pi^{0} \rightarrow \pi^{+} \pi^{-} \pi^{0}\right)}{B R\left(f_{1}(1285) \rightarrow a_{0}^{0}(980) \pi^{0} \rightarrow \eta \pi^{0} \pi^{0}\right)} \\
=(2.5 \pm 0.9) \% .
\end{gathered}
$$

4.2. BESIII experiment in Beijing: reactions $J / \psi \rightarrow \phi f_{0}(\mathbf{9 8 0}) \rightarrow \phi a_{0}(\mathbf{9 8 0}) \rightarrow \phi \eta \pi^{0}$ and $\chi_{c 1} \rightarrow a_{0}(980) \pi^{0} \rightarrow f_{0}(980) \pi^{0} \rightarrow \pi^{+} \pi^{-} \pi^{0}$

A proposal was made in [123] to search for $a_{0}^{0}(980)-$ $f_{0}(980)$ mixing in the reaction $J / \psi \rightarrow \phi f_{0}(980) \rightarrow$ $\phi a_{0}(980) \rightarrow \phi \eta \pi^{0}$, which as estimates show can be measured at the Beijing-based upgraded electron+positron collider using the BES III detector. It was later proposed to use the same facility to search for the $a_{0}^{0}(980)-f_{0}(980)$ mixing in the reaction $\chi_{c 1} \rightarrow a_{0}(980) \pi^{0} \rightarrow f_{0}(980) \pi^{0} \rightarrow$ $\pi^{+} \pi^{-} \pi^{0}$ [124]. Corresponding measurements were performed shortly after that, among other initial experiments with the BESIII detector [96]. The rates $\xi_{f a}$ and $\xi_{a f}$ of the $f_{0}(980) \rightarrow a_{0}^{0}(980)$ and $a_{0}^{0}(980) \rightarrow f_{0}(980)$ transitions were determined as a result of those experiments:

$$
\begin{gathered}
\xi_{f a}=\frac{B R\left(J / \psi \rightarrow \phi f_{0}(980) \rightarrow \phi a_{0}^{0}(980) \rightarrow \phi \eta \pi^{0}\right)}{B R\left(J / \psi \rightarrow \phi f_{0}(980) \rightarrow \phi \pi \pi\right)} \\
=(0.60 \pm 0.20(\text { stat. }) \pm 0.12(\text { sys. }) \pm 0.26(\text { para. })) \%, \quad(34) \\
\xi_{a f}=\frac{B R\left(\chi_{c 1} \rightarrow a_{0}^{0}(980) \pi^{0} \rightarrow f_{0}(980) \pi^{0} \rightarrow \pi^{+} \pi^{-} \pi^{0}\right)}{B R\left(\chi_{c 1} \rightarrow a_{0}^{0}(980) \pi^{0} \rightarrow \eta \pi^{0} \pi^{0}\right)} \\
=(0.31 \pm 0.16(\text { stat. }) \pm 0.14(\text { sys. }) \pm 0.03(\text { para. })) \%,(35)
\end{gathered}
$$

where the abbreviations stat., syst., and param. mean statistical, systematic, and parameterization-related errors. The values of the denominators in (34) and (35) where respectively taken from [96] and [144, 145].

Enhancing the accuracy of the data in (34) and (35) obtained by the BESIII collaboration in the first experiments for reactions (25) and (26) is an interesting and challenging task.

\subsection{Data analysis}


Some specific values of the couplings to the $\pi \pi, K \bar{K}$, and $\eta \pi$ decay channels are typically used in standard calculations related to $f_{0}(980)$ and $a_{0}(980)$ resonances. We first quote the estimates for these constants obtained in 95 directly from the BES III data [96] on $a_{0}^{0}(980)-f_{0}(980)$ mixing and then proceed to discussing the unexpected consequences of the VES data [122].

Because the $a_{0}^{0}(980)-f_{0}(980)$ mixing is primarily determined by the contribution of the $K \bar{K}$ loops, we set

$$
\begin{gathered}
\xi_{f a}=\frac{B R\left(f_{0}(980) \rightarrow K \bar{K} \rightarrow a_{0}^{0}(980) \rightarrow \eta \pi^{0}\right)}{B R\left(f_{0}(980) \rightarrow \pi \pi\right)}, \\
\xi_{a f}=\frac{B R\left(a_{0}^{0}(980) \rightarrow K \bar{K} \rightarrow f_{0}(980) \rightarrow \pi^{+} \pi^{-}\right)}{B R\left(a_{0}^{0}(980) \rightarrow \eta \pi^{0}\right)},
\end{gathered}
$$

where

$$
\begin{gathered}
B R\left(f_{0}(980) \rightarrow K \bar{K} \rightarrow a_{0}^{0}(980) \rightarrow \eta \pi^{0}\right) \\
=\int\left|G_{a_{0}^{0} f_{0}}(m)\right|^{2} \frac{2 m^{2} \Gamma_{a_{0}^{0} \rightarrow \eta \pi^{0}}(m)}{\pi} d m, \\
B R\left(a_{0}^{0}(980) \rightarrow K \bar{K} \rightarrow f_{0}(980) \rightarrow \pi^{+} \pi^{-}\right) \\
=\int\left|G_{a_{0}^{0} f_{0}}(m)\right|^{2} \frac{2 m^{2} \Gamma_{f_{0} \rightarrow \pi^{+} \pi^{-}}(m)}{\pi} d m, \\
B R\left(a_{0}^{0}(980) \rightarrow \eta \pi^{0}\right)=\int \frac{2 m^{2} \Gamma_{a_{0}^{0} \rightarrow \eta \pi^{0}}(m)}{\pi\left|D_{a_{0}^{0}}(m)\right|^{2}} d m, \\
B R\left(f_{0}(980) \rightarrow \pi \pi\right)=\int \frac{2 m^{2} \Gamma_{f_{0} \rightarrow \pi \pi}(m)}{\pi\left|D_{f_{0}}(m)\right|^{2}} d m,
\end{gathered}
$$

[see also Eqns (4)-(9)]. ${ }^{3}$

Substituting the central values of $\xi_{f a}$ and $\xi_{a f}$ from (34) and (35) in the left-hand sides of Eqns (36) and (37), we obtain equations for the coupling constants of $a_{0}^{0}(980)$ and $f_{0}(980)$ resonances, which must be solved numerically. Proceeding in this way, we found the following approximate values in [95]:

$$
\frac{g_{f_{0} \pi \pi}^{2}}{16 \pi} \equiv \frac{3}{2} \frac{g_{f_{0} \pi^{+} \pi^{-}}^{2}}{16 \pi}=0.098 \mathrm{GeV}^{2},
$$

\footnotetext{
${ }^{3}$ In passing from (34) and (35) to (36) and (37), we disregard the dependence of the kinematic factors contained in the $J / \psi \rightarrow \phi f_{0}(980)$ and $\chi_{c 1} \rightarrow a_{0}^{0}(980) \pi^{0}$ vertices on the invariant virtual masses of scalar mesons, because it is not significant in the decays of heavy $J / \psi(3097)$ and $\chi_{c 1}(3511)$ resonances. The values of the virtual masses were taken to be those of the scalar mesons. The corresponding kinematic factors in the equations above simply drop out under this approximation. We also disregard the interference of the contributions to the $\chi_{c 1} \rightarrow a_{0}^{0}(980) \pi^{0} \rightarrow \eta \pi^{0} \pi^{0}$ decay related to the permutation of identical $\pi^{0}$ mesons. This interference is small because the relative momenta of the $a_{0}^{0}(980)$ and $\pi^{0}$ mesons in the decay $\chi_{c 1}(3510) \rightarrow a_{0}^{0}(980) \pi^{0}$ are large.
}

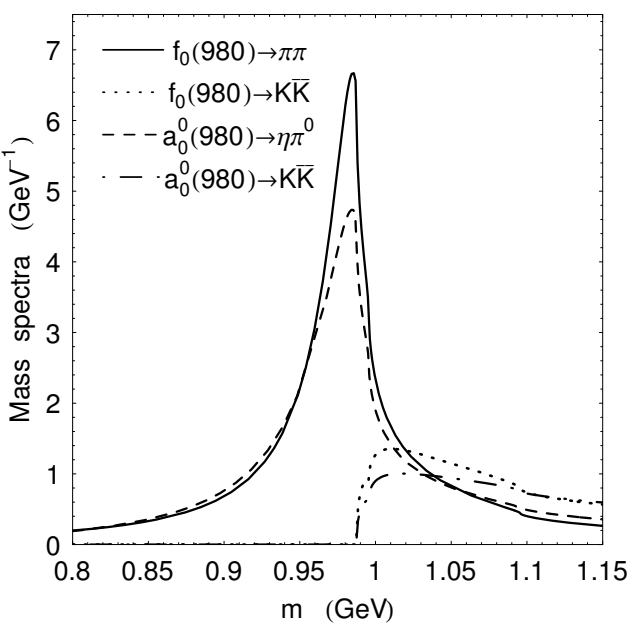

Figure 6: Mass spectra in isospin-conserving decays of $f_{0}(980)$ and $a_{0}^{0}(980)$ resonances.

$$
\begin{gathered}
\frac{g_{f_{0} K \bar{K}}^{2}}{16 \pi} \equiv 2 \frac{g_{f_{0} K^{+} K^{-}}^{2}}{16 \pi}=0.4 \mathrm{GeV}^{2}, \\
\frac{g_{a_{0}^{0} \eta \pi^{0}}^{2}}{16 \pi}=0.2 \mathrm{GeV}^{2}, \\
\frac{g_{a_{0}^{0} K \bar{K}}^{2}}{16 \pi} \equiv 2 \frac{g_{a_{0}^{0} K^{+} K^{-}}^{2}}{16 \pi}=0.5 \mathrm{GeV}^{2} .
\end{gathered}
$$

The masses of $a_{0}^{0}(980)$ and $f_{0}(980)$ resonances, $m_{a_{0}^{0}}=$ $0.985 \mathrm{GeV}$ and $m_{f_{0}}=0.985 \mathrm{GeV}$, were kept fixed in this procedure, and for the constants of their coupling to the $\eta^{\prime} \pi^{0}$ and $\eta \eta$ channels we used the relations that follow from the $q^{2} \bar{q}^{2}$ model: $g_{a_{0}^{0} \eta^{\prime} \pi^{0}}^{2}=g_{a_{0}^{0} \eta \pi^{0}}^{2}$ and $g_{f_{0} \eta \eta}^{2}=$ $g_{f_{0} K^{+} K^{-}}^{2}$ (see, e.g., [3, 18]). Integration in (38) and (39) included the region from $0.9 \mathrm{GeV}$ to $1.05 \mathrm{GeV}$ (see Fig. 3 ) and, in (40) and (41), from the respective $\eta \pi^{0}$ and $\pi \pi$ threshold to $1.3 \mathrm{GeV}$.

These are the values of the constants that we used in Section 2 to obtain the absolute value of the $a_{0}^{0}(980)-$ $f_{0}(980)$ transition in Fig. 2, the mass spectra for isospinviolating decays of $f_{0}(980)$ and $a_{0}^{0}(980)$ resonances in Fig. 3 , and estimates (3), (10), and (11). The mass spectra of the isospin-preserving decays of $f_{0}(980)$ and $a_{0}^{0}(980)$ resonances as a function of $m$ calculated for the obtained values of the constants are displayed in Fig. 6. The curves plotted there represent the integrands in (40) and (41) and similar expressions for the mass spectra in the decays into $K \bar{K}$. There is nothing special in these spectra.

There are many studies in which the constants of the $f_{0}(980)$ and $a_{0}(980)$ coupling to the $\pi \pi, K \bar{K}$, and $\eta \pi$ channels have been calculated, estimated, and determined by fitting (see, e.g., [2, 3, 12 15, 18, 43, 58, 67, 97, 104, 105, 123, 124], this list is far from complete). The obtained values of the constants are dispersed in a rather broad range. The values of the constants determined in different ways and from various reactions often disagree 
by a factor of two and sometimes even more. The values in (42)-(45) fall somewhere in the middle of the range of those available in the literature; in our opinion, it is natural to use them as a guide in making estimates.

As regards the decay $f_{1}(1285) \rightarrow f_{0}(980) \pi^{0} \rightarrow$ $\pi^{+} \pi^{-} \pi^{0}$, the VES data [122] [see (33)] require a 'huge' $a_{0}^{0}(980)-f_{0}(980)$ mixing:

$$
\begin{gathered}
\frac{B R\left(f_{1}(1285) \rightarrow a_{0}^{0}(980) \pi^{0} \rightarrow f_{0}(980) \pi^{0} \rightarrow \pi^{+} \pi^{-} \pi^{0}\right)}{B R\left(f_{1}(1285) \rightarrow a_{0}^{0}(980) \pi^{0} \rightarrow \eta \pi^{0} \pi^{0}\right)} \\
=(2.5 \pm 0.9) \% \\
\approx \frac{B R\left(a_{0}^{0}(980) \rightarrow K \bar{K} \rightarrow f_{0}(980) \rightarrow \pi^{+} \pi^{-}\right)}{B R\left(a_{0}^{0}(980) \rightarrow \eta \pi^{0}\right)}
\end{gathered}
$$

[compare (46) with (35) and (37)] and, as a consequence, the constants of the $f_{0}(980)$ and $a_{0}^{0}(980)$ resonance coupling to pseudoscalar mesons that are unsatisfactory in many aspects:

$$
\begin{aligned}
\frac{g_{f_{0} \pi \pi}^{2}}{16 \pi} \equiv & \frac{3}{2} \frac{g_{f_{0} \pi^{+} \pi^{-}}^{2}}{16 \pi}=0.46 \mathrm{GeV}^{2} \\
\frac{g_{f_{0} K \bar{K}}^{2}}{16 \pi} \equiv & 2 \frac{g_{f_{0} K^{+} K^{-}}^{2}}{16 \pi}=2.87 \mathrm{GeV}^{2} \\
& \frac{g_{a_{0}^{0} \eta \pi^{0}}^{2}}{16 \pi}=0.48 \mathrm{GeV}^{2}, \\
\frac{g_{a_{0}^{0} K \bar{K}}^{2}}{16 \pi} \equiv & 2 \frac{g_{a_{0}^{0} K^{+} K^{-}}^{2}}{16 \pi}=4.97 \mathrm{GeV}^{2}
\end{aligned}
$$

For example, due to the very large values of $g_{f_{0} K \bar{K}}^{2} /(16 \pi)$ and $g_{a_{0}^{0} K \bar{K}}^{2} /(16 \pi)$ in (48) and (50), the width of the $a_{0}^{0}(980)$ resonance in the $\eta \pi^{0}$ mass spectrum only proves to be about $15 \mathrm{MeV}$, and the value of $\xi_{a f}$ calculated using Eqn (37) is about nine times larger than its central experimental value in (35).

The ratio

$$
\frac{B R\left(f_{1}(1285) \rightarrow a_{0}^{0}(980) \pi^{0} \rightarrow f_{0}(980) \pi^{0} \rightarrow \pi^{+} \pi^{-} \pi^{0}\right)}{B R\left(f_{1}(1285) \rightarrow a_{0}^{0}(980) \pi^{0} \rightarrow \eta \pi^{0} \pi^{0}\right)}
$$

calculated using the set of constants in (42)-(45) is [95 $]^{4}$

$$
\begin{gathered}
\frac{B R\left(f_{1}(1285) \rightarrow a_{0}^{0}(980) \pi^{0} \rightarrow f_{0}(980) \pi^{0} \rightarrow \pi^{+} \pi^{-} \pi^{0}\right)}{B R\left(f_{1}(1285) \rightarrow a_{0}^{0}(980) \pi^{0} \rightarrow \eta \pi^{0} \pi^{0}\right)} \\
\approx 0.29 \%
\end{gathered}
$$

This ratio is close to the central value of $\xi_{a f}$ in (35); however, it is approximately one order of magnitude

\footnotetext{
${ }^{4}$ Because the $f_{1}(1285)$ resonance, whose mass is $m_{f_{1}}=(1281.9 \pm$ $0.5) \mathrm{MeV}$ [9], is located only $160 \mathrm{MeV}$ higher than the nominal $a_{0}(980) \pi$-production threshold, the calculations took both the $P$-wave character of the $f_{1}(1285) \rightarrow a_{0}(980) \pi$ decays and the interference of two amplitudes in the $f_{1}(1285) \rightarrow a_{0}(980) \pi \rightarrow$ $\eta \pi \pi$ channel into account. More details can be found in Section 5 .
}

smaller than the value required to explain the VES data 122] [see (33)]. Thus, we can conclude that the VES collaboration 122] has discovered a significant violation of isotopic symmetry in the region of $a_{0}^{0}(980)$ and $f_{0}(980)$ resonances, which can hardly be explained by the $a_{0}^{0}(980)-f_{0}(980)$ mixing alone.

In Section 5, following experimental indications, we consider additional $K \bar{K}$-loop mechanisms of the $f_{1}(1285) \rightarrow f_{0}(980) \pi^{0} \rightarrow \pi^{+} \pi^{-} \pi^{0}$ decay that are due, similarly to the $a_{0}^{0}(980)-f_{0}(980)$ mixing, to the mass difference of $K^{+}$- and $K^{0}$ mesons. We also use the decay $f_{1}(1285) \rightarrow f_{0}(980) \pi^{0} \rightarrow \pi^{+} \pi^{-} \pi^{0}$ to demonstrate the general approach to estimating the total contribution of the $K \bar{K}$-loop mechanisms that violate isotopic invariance.

\section{Strong violation of isotopic invariance according to BESIII data for the reactions $J / \psi \rightarrow \phi f_{1}(1285) \rightarrow \phi f_{0}(980) \pi^{0} \rightarrow \phi 3 \pi$ and $J / \psi \rightarrow \gamma \eta(1405) \rightarrow \gamma f_{0}(980) \pi^{0} \rightarrow$ $\gamma 3 \pi$}

\subsection{Mechanisms of $f_{1}(1285) \rightarrow f_{0}(980) \pi^{0} \rightarrow 3 \pi$ decay}

After the VES experiment [122] had been completed, the isospin-violating decay $f_{1}(1285) \rightarrow \pi^{+} \pi^{-} \pi^{0}$ was also observed by the BESIII collaboration [134] in the reaction $J / \psi \rightarrow \phi f_{1}(1285) \rightarrow \phi f_{0}(980) \pi^{0} \rightarrow \phi 3 \pi$. The experiment yielded the branching function ratio

$$
\begin{gathered}
\frac{B R\left(f_{1}(1285) \rightarrow f_{0}(980) \pi^{0} \rightarrow \pi^{+} \pi^{-} \pi^{0}\right)}{B R\left(f_{1}(1285) \rightarrow a_{0}^{0}(980) \pi^{0} \rightarrow \eta \pi^{0} \pi^{0}\right)} \\
=(3.6 \pm 1.4) \%
\end{gathered}
$$

It was also indicated that a specific feature of the $f_{1}(1285) \rightarrow \pi^{+} \pi^{-} \pi^{0}$ transition is that the $\pi^{+} \pi^{-}$mass spectrum is dominated by a narrow resonance structure located in the region of $K \bar{K}$ thresholds.

Another indication of the decay $f_{1}(1285) / \eta(1295) \rightarrow$ $\pi^{+} \pi^{-} \pi^{0}$ came from the BESIII experiment [125] combined with data on the reaction $J / \psi \rightarrow \gamma \eta(1405) \rightarrow$ $\gamma f_{0}(980) \pi^{0} \rightarrow \gamma 3 \pi$. If it is considered to be due to $f_{1}(1285)$ alone, we obtain

$$
\begin{gathered}
\frac{B R\left(f_{1}(1285) \rightarrow f_{0}(980) \pi^{0} \rightarrow \pi^{+} \pi^{-} \pi^{0}\right)}{B R\left(f_{1}(1285) \rightarrow a_{0}^{0}(980) \pi^{0} \rightarrow \eta \pi^{0} \pi^{0}\right)} \\
=(1.3 \pm 0.7) \% .
\end{gathered}
$$

Thus, according to data from the first experiments (33), (52), and (53), the ratio of the isospin-forbidden decay $f_{1}(1285) \rightarrow f_{0}(980) \pi^{0} \rightarrow \pi^{+} \pi^{-} \pi^{0}$ and the isospinallowed decay $f_{1}(1285) \rightarrow a_{0}^{0}(980) \pi^{0} \rightarrow \eta \pi^{0} \pi^{0}$ can be as large as one to four percent. This value is too high for a quantity that is apparently expected to be of the order of $10^{-4}$. The data rather convincingly suggest the existence of mechanisms that enhance the $f_{1}(1285) \rightarrow \pi^{+} \pi^{-} \pi^{0}$ decay. Nevertheless, they still need to be confirmed. Below, in comparing theoretical estimates with experimental data, for certainty we use the VES data [122] [see (33)] as mean values. 


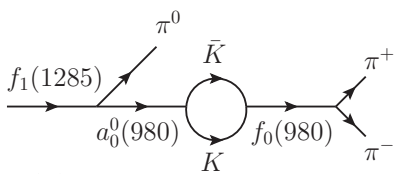

(1)

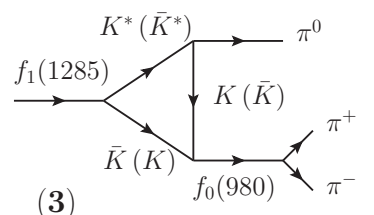

(3)

Figure 7: Diagrams corresponding to the possible $K \bar{K}$-loop mechanisms of the decay $f_{1}(1285) \rightarrow f_{0}(980) \pi^{0} \rightarrow \pi^{+} \pi^{-} \pi^{0}$.

We now proceed to estimating the contribution of the following possible $K \bar{K}$-loop mechanisms to the decay $f_{1}(1285) \rightarrow f_{0}(980) \pi^{0} \rightarrow \pi^{+} \pi^{-} \pi^{0}$ in detail:

(1) the $a_{0}^{0}(980)-f_{0}(980)$ mixing, $f_{1}(1285) \rightarrow$ $a_{0}(980) \pi^{0} \rightarrow\left(K^{+} K^{-}+K^{0} \bar{K}^{0}\right) \pi^{0} \rightarrow f_{0}(980) \pi^{0} \rightarrow$ $\pi^{+} \pi^{-} \pi^{0}$

(2) the transition $f_{1}(1285) \rightarrow\left(K^{+} K^{-}+K^{0} \bar{K}^{0}\right) \pi^{0} \rightarrow$ $f_{0}(980) \pi^{0} \rightarrow \pi^{+} \pi^{-} \pi^{0}$, which is due to the pointlike $f_{1}(1285) \rightarrow K \bar{K} \pi^{0}$ decay;

(3) the transition $f_{1}(1285) \rightarrow\left(K^{*} \bar{K}+\bar{K}^{*} K\right) \rightarrow$ $\left(K^{+} K^{-}+K^{0} \bar{K}^{0}\right) \pi^{0} \rightarrow f_{0}(980) \pi^{0} \rightarrow \pi^{+} \pi^{-} \pi^{0}$, where $K^{*}=K^{*}(892)$;

(4) the transition $f_{1}(1285) \rightarrow\left(K_{0}^{*} \bar{K}+\bar{K}_{0}^{*} K\right) \rightarrow$ $\left(K^{+} K^{-}+K^{0} \bar{K}^{0}\right) \pi^{0} \rightarrow f_{0}(980) \rightarrow \pi^{+} \pi^{-} \pi^{0}$, where $K_{0}^{*}=K_{0}^{*}(800)($ or $\kappa)$ and $K_{0}^{*}(1430)$.

Figure 7 illustrates the specified mechanisms in terms of respective diagrams (1)-(4). The $f_{1}(1285$ meson first decays within each of these mechanisms into $K \bar{K} \pi^{0}$ (according to the data in [9], the decay $f_{1}(1285) \rightarrow$ $K \bar{K} \pi$ into all charge modes occurs in about $9 \%$ of cases). Next, owing to the final-state interaction between $K$ and $\bar{K}$ mesons, i.e., the transitions $K^{+} K^{-} \rightarrow$ $f_{0}(980) \rightarrow \pi^{+} \pi^{-}$and $K^{0} \bar{K}^{0} \rightarrow f_{0}(980) \rightarrow \pi^{+} \pi^{-}$, the isospin-violating decay $f_{1}(1285) \rightarrow\left(K^{+} K^{-}+\right.$ $\left.K^{0} \bar{K}^{0}\right) \pi^{0} \rightarrow f_{0}(980) \pi^{0} \rightarrow \pi^{+} \pi^{-} \pi^{0}$ is initiated (see Fig. $7)$. This occurs because the contributions from the production of $K^{+} K^{-}$and $K^{0} \bar{K}^{0}$ pairs fail to fully cancel each other due to the mass difference between $K^{+}$ and $K^{0}$ mesons. This mutual compensation is naturally minimal in the m region between the $K^{+} K^{-}$and $K^{0} \bar{K}^{0}$ thresholds. Regardless of the specific mechanism of $K \bar{K}$-pair production, this results in the emergence in the $\pi^{+} \pi^{-}$mass spectrum of a narrow resonance structure located in the vicinity of the $K \bar{K}$ thresholds, with the width $\approx 2 m_{K^{0}}-2 m_{K^{+}} \approx 8 \mathrm{MeV}$. Observing this structure in an experiment would be a direct indication of the general $K \bar{K}$-loop mechanism in isotopic invariance violation.

(1) The main estimate that characterizes the contribution of $a_{0}^{0}(980)-f_{0}(980)$ mixing to the decay $f_{1}(1285) \rightarrow$ $f_{0}(980) \pi^{0} \rightarrow \pi^{+} \pi^{-} \pi^{0}$ is already presented in Eqn (51) (see also the discussion that follows the formula), and we only elucidate some details of that calculation [95].

First, we present the expression for the width of the $f_{1}(1285) \rightarrow a_{0}^{0}(980) \pi^{0} \rightarrow \eta \pi^{0} \pi^{0}$ decay:

$$
=\frac{g_{f_{1} a_{0}^{0} \pi^{0}}^{2} g_{a_{0}^{0} \eta \pi^{0}}^{2}}{192 \pi^{3} m_{f_{1}}^{3}} \int_{\left(m_{\eta}+m_{\pi}\right)^{2}}^{\Gamma_{f_{1} \rightarrow a_{0}^{0} \pi^{0} \rightarrow \eta \pi^{0} \pi^{0}}} d s \int_{a_{-}(s)}^{\left(m_{f_{1}}-m_{\pi}\right)^{2}} d t \mathcal{T}(s, t),
$$

where $s(t)$ is the invariant mass squared of the $\eta \pi_{1}^{0}\left(\eta \pi_{2}^{0}\right)$ pair in the decay $f_{1}(1285) \rightarrow \eta \pi_{1}^{0} \pi_{2}^{0}$,

$$
\begin{gathered}
\mathcal{T}(s, t)=\frac{p^{2}(s)}{\left|D_{a_{0}^{0}}(\sqrt{s})\right|^{2}}+\operatorname{Re} \frac{p(s) p(t) \cos \theta}{D_{a_{0}^{0}}(\sqrt{s}) D_{a_{0}^{0}}^{*}(\sqrt{t})} \\
a_{ \pm}(s)=\frac{1}{2}\left(m_{f_{1}}^{2}+m_{\eta}^{2}+2 m_{\pi}^{2}-s\right) \\
+\frac{\left(m_{f_{1}}^{2}-m_{\pi}^{2}\right)\left(m_{\eta}^{2}-m_{\pi}^{2}\right)}{2 s} \pm \frac{2 m_{f_{1}}}{\sqrt{s}} p(s) q(s)
\end{gathered}
$$

$$
\begin{gathered}
p(s)=\sqrt{m_{f_{1}}^{4}-2 m_{f_{1}}^{2}\left(s+m_{\pi}^{2}\right)+\left(s-m_{\pi}^{2}\right)^{2}} /\left(2 m_{f_{1}}\right),(57) \\
p(t)=\sqrt{m_{f_{1}}^{4}-2 m_{f_{1}}^{2}\left(t+m_{\pi}^{2}\right)+\left(t-m_{\pi}^{2}\right)^{2}} /\left(2 m_{f_{1}}\right),(58) \\
q(s)=\sqrt{s^{2}-2 s\left(m_{\eta}^{2}+m_{\pi}^{2}\right)+\left(m_{\eta}^{2}-m_{\pi}^{2}\right)^{2}} /(2 \sqrt{s}),(59) \\
p(s) p(t) \cos \theta=\frac{1}{2}\left(s+t-m_{f_{1}}^{2}-m_{\eta}^{2}\right) \\
+\frac{\left(m_{f_{1}}^{2}+m_{\pi}^{2}-s\right)\left(m_{f_{1}}^{2}+m_{\pi}^{2}-t\right)}{4 m_{f_{1}}^{2}}
\end{gathered}
$$

As an effective vertex of the $f_{1}(1285) a_{0}^{0}(980) \pi^{0}$ coupling, we here used the expression $V_{f_{1} a_{0}^{0} \pi^{0}}=g_{f_{1} a_{0}^{0} \pi^{0}}\left(\epsilon_{f_{1}}, p_{\pi^{0}}-\right.$ $\left.p_{a_{0}^{0}}\right)$, where $\epsilon_{f_{1}}$ is the $f_{1}(1285)$ polarization 4 -vector and $p_{\pi^{0}}$ and $p_{a_{0}^{0}}$ are the 4-momenta of $\pi^{0}$ and $a_{0}^{0}(980)$. If isotopic invariance holds, then $\Gamma_{f_{1} \rightarrow a_{0}^{0} \pi^{0} \rightarrow \eta \pi^{0} \pi^{0}}=$ $\Gamma_{f_{1} \rightarrow a_{0} \pi \rightarrow \eta \pi \pi} / 3$.

Similarly, we have the width of the $f_{1}(1285) \rightarrow$ $a_{0}(980) \pi^{0} \rightarrow K \bar{K} \pi$ decay

$$
\begin{gathered}
\Gamma_{f_{1} \rightarrow a_{0} \pi \rightarrow K \bar{K} \pi}=6 \Gamma_{f_{1} \rightarrow a_{0}^{0} \pi^{0} \rightarrow K^{+} K^{-} \pi^{0}} \\
=\frac{g_{f_{1} a_{0}^{0} \pi^{0}}^{2}}{\pi m_{f_{1}}^{2}} \int_{2 m_{K^{+}}}^{m_{f_{1}}-m_{\pi^{0}}} p^{3}\left(m^{2}\right) \frac{2 m^{2} \Gamma_{a_{0}^{0} \rightarrow K^{+} K^{-}}(m)}{\pi\left|D_{a_{0}^{0}}(m)\right|^{2}} d m .
\end{gathered}
$$

The width of the decay $f_{1}(1285) \rightarrow a_{0}^{0}(980) \pi^{0} \rightarrow$ $f_{0}(980) \pi^{0} \rightarrow \pi^{+} \pi^{-} \pi^{0}$ due to the $a_{0}^{0}(980)-f_{0}(980)$ mixing (see diagram (1) in Fig. 7) is

$$
\begin{gathered}
\Gamma_{f_{1} \rightarrow a_{0}^{0} \pi^{0} \rightarrow f_{0} \pi^{0} \rightarrow \pi^{+} \pi^{-} \pi^{0}}=\frac{g_{f_{1} a_{0}^{0} \pi^{0}}^{2}}{6 \pi m_{f_{1}}^{2}} \\
\times \int_{0.9 \mathrm{GeV}}^{1.05 \mathrm{GeV}}\left|G_{a_{0}^{0} f_{0}}(m)\right|^{2} p^{3}\left(m^{2}\right) \frac{2 m^{2} \Gamma_{f_{0} \rightarrow \pi^{+} \pi^{-}}(m)}{\pi} d m .(62)
\end{gathered}
$$


The shape of the $\pi^{+} \pi^{-}$mass spectrum that follows from the integrand in (62) is virtually undistinguishable from the curves shown in Fig. 3.

Numerical integration in (54) and (62) yields

$$
\begin{gathered}
\frac{\Gamma_{f_{1} \rightarrow a_{0}^{0} \pi^{0} \rightarrow f_{0} \pi^{0} \rightarrow \pi^{+} \pi^{-} \pi^{0}}}{\Gamma_{f_{1} \rightarrow a_{0}^{0} \pi^{0} \rightarrow \eta \pi^{0} \pi^{0}}} \\
=\frac{B R\left(f_{1} \rightarrow a_{0}^{0}(980) \pi^{0} \rightarrow f_{0}(980) \pi^{0} \rightarrow \pi^{+} \pi^{-} \pi^{0}\right)}{B R\left(f_{1} \rightarrow a_{0}^{0}(980) \pi^{0} \rightarrow \eta \pi^{0} \pi^{0}\right)} \\
\approx 0.29 \% .
\end{gathered}
$$

This result was discussed after Eqn (51). From (54) and (61), we also obtain

$$
\begin{aligned}
\frac{\Gamma_{f_{1} \rightarrow a_{0} \pi \rightarrow K \bar{K} \pi}}{\Gamma_{f_{1} \rightarrow a_{0} \pi \rightarrow \eta \pi \pi}}= & \frac{B R\left(f_{1} \rightarrow a_{0}(980) \pi \rightarrow K \bar{K} \pi\right)}{B R\left(f_{1} \rightarrow a_{0}(980) \pi \rightarrow \eta \pi \pi\right)} \\
& \approx 0.11 .
\end{aligned}
$$

It is noteworthy that the PDG data [9] for the ratio

$$
\frac{B R\left(f_{1} \rightarrow K \bar{K} \pi\right)}{B R\left(f_{1} \rightarrow a_{0}(980) \pi \rightarrow \eta \pi \pi\right)} \approx 0.25 \pm 0.05
$$

are not in disagreement with (64) and indicate that the transition $f_{1}(1285) \rightarrow a_{0}(980) \pi \rightarrow K \bar{K} \pi$ may not be the single source of the $f_{1}(1285) \rightarrow K \bar{K} \pi$ decay.

(2) A detailed calculation for the transition $f_{1}(1285) \rightarrow$ $\left(K^{+} K^{-}+K^{0} \bar{K}^{0}\right) \pi^{0} \rightarrow f_{0}(980) \pi^{0} \rightarrow \pi^{+} \pi^{-} \pi^{0}$, which is due to the pointlike decay $f_{1}(1285) \rightarrow K \bar{K} \pi^{0}$ (see diagram (2) in Fig. 7), yields 95]

$$
\frac{\Gamma_{f_{1} \rightarrow\left(K^{+} K^{-}+K^{0} \bar{K}^{0}\right) \pi^{0} \rightarrow f_{0} \pi^{0} \rightarrow \pi^{+} \pi^{-} \pi^{0}}}{\Gamma_{f_{1} \rightarrow K \bar{K} \pi}} \approx 0.0022 .
$$

This result is almost 15 times smaller than the corresponding experimental value

$$
\begin{gathered}
\frac{B R\left(f_{1}(1285) \rightarrow f_{0}(980) \pi^{0} \rightarrow \pi^{+} \pi^{-} \pi^{0}\right)}{B R\left(f_{1}(1285) \rightarrow K \bar{K} \pi\right)} \\
=0.033 \pm 0.010
\end{gathered}
$$

(which contains a significant error, however) following from the VES [122] [see (32)] and PDG 9] data.

The $\pi^{+} \pi^{-}$mass spectrum in the decay $f_{1}(1285) \rightarrow$ $\left(K^{+} K^{-}+K^{0} \bar{K}^{0}\right) \pi^{0} \rightarrow f_{0}(980) \pi^{0} \rightarrow \pi^{+} \pi^{-} \pi^{0}$ is, for this mechanism, similar to the curves in Fig. 3. It is clear, however, that the pointlike mechanism of the decay $f_{1}(1285) \rightarrow K \bar{K} \pi$ cannot per se yield a significant probability of the decay $f_{1}(1285) \rightarrow f_{0}(980) \pi^{0} \rightarrow \pi^{+} \pi^{-} \pi^{0}$.

(3) We now proceed to discussing the mechanism of the decay $f_{1}(1285) \rightarrow\left(K^{*} \bar{K}+\bar{K}^{*} K\right) \rightarrow\left(K^{+} K^{-}+\right.$ $\left.K^{0} \bar{K}^{0}\right) \pi^{0} \rightarrow f_{0}(980) \pi^{0} \rightarrow \pi^{+} \pi^{-} \pi^{0}$ [where $K^{*}=$ $\left.K^{*}(892)\right]$ induced by diagram (3) in Fig. 7 .

The widths of the decays $\Gamma\left(f_{1}(1285) \rightarrow\left(K^{*} \bar{K}+\right.\right.$ $\left.\left.\bar{K}^{*} K\right) \rightarrow K \bar{K} \pi\right)$ and $\Gamma\left(f_{1}(1285) \rightarrow\left(K^{*} \bar{K}+\bar{K}^{*} K\right) \rightarrow\right.$ $\left.\left(K^{+} K^{-}+K^{0} \bar{K}^{0}\right) \pi^{0} \rightarrow f_{0}(980) \pi^{0} \rightarrow \pi^{+} \pi^{-} \pi^{0}\right)$ are described in this case by simple but rather cumbersome formulas. We do not present them here (the interested reader can find them in 95]), but only briefly discuss the assumptions made in the calculations and report the calculation results.

Figure 8 shows a diagram that corresponds to the transition $f_{1}(1285) \rightarrow\left(K^{*} \bar{K}+\bar{K}^{*} K\right) \rightarrow K \bar{K} \pi$ (the caption to Fig. 8 specifies the notation for the particles involved in the decay.) The $f_{1}(1285) \rightarrow K^{*} \bar{K}$ vertex is generically determined by two independent effective coupling constants. Given today's state of experimental data, the general structure of this vertex is de facto unknown. Therefore, for certainty, we limit our approach to a partial expression (in the spirit of effective chiral Lagrangians [146 148]) of the form

$$
V_{f_{1} K^{*} \bar{K}}=g_{f_{1} K^{*} \bar{K}} F_{\mu \nu}^{\left(f_{1}\right)} F^{\left(K^{*}\right) \mu \nu},
$$

where $F_{\mu \nu}^{\left(f_{1}\right)}=p_{1 \mu} \epsilon_{f_{1} \nu}-p_{1 \nu} \epsilon_{f_{1} \mu}, F_{\mu \nu}^{\left(K^{*}\right)}=k_{1 \mu} \epsilon_{K^{*} \nu}-$ $k_{1 \nu} \epsilon_{K^{*} \mu}, \epsilon_{f_{1}}$ and $\epsilon_{K^{*}}$ are the respective polarization 4vectors of $f_{1}(1285)$ and $K^{*}$ mesons. It is noteworthy

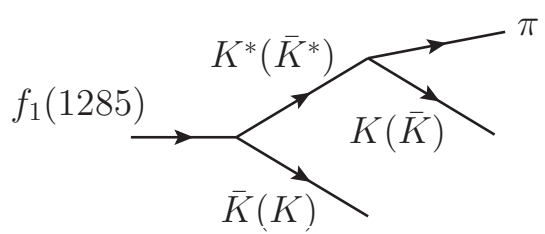

Figure 8: Diagram of the decay $f_{1}(1285) \rightarrow\left(K^{*} \bar{K}+\bar{K}^{*} K\right) \rightarrow$ $K \bar{K} \pi$. The respective 4-momenta of $f_{1}(1285), K(\bar{K}), K^{*}$ $\left(\bar{K}^{*}\right)$, and $\pi$ are $p_{1}, p_{K}\left(p_{\bar{K}}\right), k_{1}\left(k_{2}\right)$, and $p_{\pi}$.

that the $K^{*}$ resonance produced as a result of such a transverse interaction carries spin 1 off the mass shell. The amplitude of the $K^{*} \rightarrow K \pi$ decay is

$$
V_{K^{*} K \pi}=g_{K^{*} K \pi}\left(\epsilon_{K^{*}}, p_{\pi}-p_{K}\right),
$$

where $g_{K^{*+} K^{+} \pi^{0}}=-g_{\bar{K}^{* 0} \bar{K}^{0} \pi^{0}}$ and $g_{K^{*+} K^{0} \pi^{+}}=$ $\sqrt{2} g_{K^{*+} K^{+} \pi^{0}}$. Similar formulas hold for the decays $f_{1}(1285) \rightarrow \bar{K}^{*} K$ and $\bar{K}^{*} \rightarrow \bar{K} \pi$.

As follows from Eqns (68) and (69), the product of the vertices in the amplitude corresponding to diagram (3) in Fig. 7 is of the third power in momenta. However, two momenta of the three are the momenta of external particles, and therefore the diagram converges. We note that this diagram in the physical region of the $f_{1}(1285) \rightarrow f_{0}(980) \pi^{0} \rightarrow \pi^{+} \pi^{-} \pi^{0}$ decay does not contain the logarithmic (triangular) singularity (all three particles in the loop cannot be on the mass shell simultaneously $)^{5}$ As a result, the nonzero width of the $K^{*}(892)$ resonance affects the calculation of

$$
\Gamma\left(f_{1}(1285) \rightarrow\left(K^{*} \bar{K}+\bar{K}^{*} K\right) \rightarrow K \bar{K} \pi\right)
$$

\footnotetext{
${ }^{5}$ Indeed, the invariant mass of the $K \pi$ pair $\left(k_{1}^{2}\right)^{1 / 2}$ in the decay $f_{1}(1285) \rightarrow K \bar{K} \pi$ ranges from $629 \mathrm{MeV}$ to $788 \mathrm{MeV}$, while the mass of the $K^{*}$ resonance is $m_{K^{*}} \approx 895 \mathrm{MeV}$ and its decay width is $\Gamma_{K^{*}} \approx 50 \mathrm{MeV}$.
} 


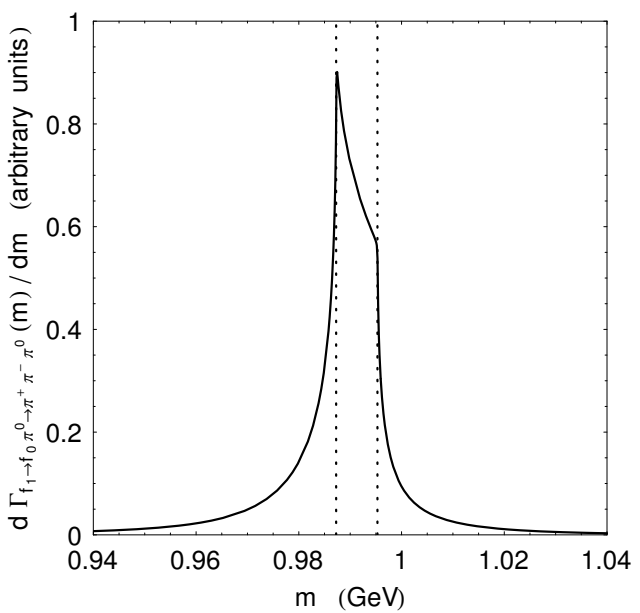

Figure 9: The $\pi^{+} \pi^{-}$mass spectrum in the decay $f_{1}(1285) \rightarrow$ $\left(K^{*} \bar{K}+\bar{K}^{*} K\right) \rightarrow\left(K^{+} K^{-}+K^{0} \bar{K}^{0}\right) \pi^{0} \rightarrow f_{0}(980) \pi^{0} \rightarrow$ $\pi^{+} \pi^{-} \pi^{0}$.

and

$$
\begin{aligned}
\Gamma\left(f_{1}(1285) \rightarrow\left(K^{*} \bar{K}+\bar{K}^{*} K\right)\right. & \rightarrow\left(K^{+} K^{-}+K^{0} \bar{K}^{0}\right) \pi^{0} \\
\rightarrow f_{0}(980) \pi^{0} & \left.\rightarrow \pi^{+} \pi^{-} \pi^{0}\right)
\end{aligned}
$$

in a negligible way.

In obtaining numerical estimates in 95$]$, the contribution to the propagator $1 / D_{K^{*}}\left(k_{1(2)}^{2}\right)=1 /\left(m_{K^{*}}^{2}-k_{1(2)}^{2}-\right.$ $\left.i m_{K^{*}} \Gamma_{K^{*}}\right)$ proportional to $\Gamma_{K^{*}}$ was simply ignored.

Figure 9 shows the $\pi^{+} \pi^{-}$mass spectrum that corresponds to the mechanism of the transition $f_{1}(1285) \rightarrow$ $\left(K^{*} \bar{K}+\bar{K}^{*} K\right) \rightarrow\left(K^{+} K^{-}+K^{0} \bar{K}^{0}\right) \pi^{0} \rightarrow f_{0}(980) \pi^{0} \rightarrow$ $\pi^{+} \pi^{-} \pi^{0}$ [95]. Its shape is virtually the same as the $\pi^{+} \pi^{-}$ mass spectrum for $a_{0}^{0}(980)-f_{0}(980)$ mixing displayed in Fig. 3. Using the $f_{1}(1285)$ decay width corresponding to the diagram in Fig. 8 and diagram (3) in Fig. 7, we integrate numerically to obtain [95]

$$
\begin{aligned}
& \Gamma_{f_{1} \rightarrow\left(K^{*} \bar{K}+\bar{K}^{*} K\right) \rightarrow K \bar{K} \pi}=\frac{g_{f_{1} K^{*+} K^{-}}^{2} g_{K^{*+} K^{+} \pi^{0}}^{2}}{4 \pi^{3}} \\
& \times 0.976 \times 10^{-2} \mathrm{GeV}^{3} \\
& \Gamma_{f_{1} \rightarrow f_{0} \pi^{0} \rightarrow \pi^{+} \pi^{-} \pi^{0}}=\frac{g_{f_{1} K^{*+} K^{-}}^{2} g_{K^{*+} K^{+} \pi^{0}}^{2}}{4 \pi^{3}} \\
& \times 0.277 \times 10^{-4} \mathrm{GeV}^{3}
\end{aligned}
$$

The ratio of these values is

$$
\begin{gathered}
\frac{\Gamma_{f_{1} \rightarrow f_{0} \pi^{0} \rightarrow \pi^{+} \pi^{-} \pi^{0}}}{\Gamma_{f_{1} \rightarrow\left(K^{*} \bar{K}+\bar{K}^{*} K\right) \rightarrow K \bar{K} \pi}} \\
=\frac{B R\left(f_{1} \rightarrow f_{0} \pi^{0} \rightarrow \pi^{+} \pi^{-} \pi^{0}\right)}{B R\left(f_{1} \rightarrow\left(K^{*} \bar{K}+\bar{K}^{*} K\right) \rightarrow K \bar{K} \pi\right)} \\
=0.284 \times 10^{-2} .
\end{gathered}
$$

Using this value and assuming that the entire value of $B R\left(f_{1} \rightarrow K \bar{K} \pi\right)=(9.0 \pm 0.4) \%$ [9] is due to the $f_{1} \rightarrow$
$\left(K^{*} \bar{K}+\bar{K}^{*} K\right) \rightarrow K \bar{K} \pi$ decay mode, we obtain the upper estimate

$$
B R\left(f_{1} \rightarrow f_{0} \pi^{0} \rightarrow \pi^{+} \pi^{-} \pi^{0}\right) \approx 0.255 \times 10^{-3} .
$$

This result is approximately 12 times smaller than the central experimental value (32) obtained by VES. Therefore, the single transition mechanism $f_{1}(1285) \rightarrow$ $\left(K^{*} \bar{K}+\bar{K}^{*} K\right) \rightarrow\left(K^{+} K^{-}+K^{0} \bar{K}^{0}\right) \pi^{0} \rightarrow f_{0}(980) \pi^{0} \rightarrow$ $\pi^{+} \pi^{-} \pi^{0}$ is apparently insufficient for understanding the experimental results.

(4) Finally, we consider the $f_{1}(1285) \rightarrow\left(K_{0}^{*} \bar{K}+\right.$ $\left.\bar{K}_{0}^{*} K\right) \rightarrow\left(K^{+} K^{-}+K^{0} \bar{K}^{0}\right) \pi^{0} \rightarrow f_{0}(980) \pi^{0} \rightarrow \pi^{+} \pi^{-} \pi^{0}$ decay mechanism, which involves the scalar $K_{0}^{*}$ meson. This mechanism is represented by diagram (4) in Fig. 7.

The version where the role of $K_{0}^{*}$ would be played by the existing resonance $K_{0}^{*}(800)$ (or $\kappa$ ) [9] should be disregarded. The point is that for the $\kappa$ resonance with a mass $m_{\kappa} \lesssim 800 \mathrm{MeV}$ and width $\Gamma_{\kappa} \approx 400-550 \mathrm{MeV}$ [9], the shapes of the $K \pi$ and $K \bar{K}$ mass spectra in the decay $f_{1}(1285) \rightarrow(\kappa \bar{K}+\bar{\kappa} K) \rightarrow K \bar{K} \pi$ are literally opposite to those observed in experiment [149 151]. According to the data on the $f_{1}(1285) \rightarrow K \bar{K} \pi$ decay [9, 149 151], a significant bump near the $K \bar{K}$ threshold is observed in the $K \bar{K}$ spectrum, and a significant bump in the $K \pi$ spectrum is observed near its upper edge, i.e., close to $m_{f_{1}}-m_{K} \approx 788 \mathrm{MeV}$. This picture agrees well with the $f_{1}(1285) \rightarrow a_{0}(980) \pi \rightarrow K \bar{K} \pi$ decay mechanism and is consistent with the $f_{1}(1285) \rightarrow\left(K^{*} \bar{K}+\bar{K}^{*} K\right) \rightarrow K \bar{K} \pi$ mechanism. However, the $f_{1}(1285) \rightarrow(\kappa \bar{K}+\bar{\kappa} K) \rightarrow$ $K \bar{K} \pi$ mechanism generates a bump near the upper end of the $K \bar{K}$ spectrum, i.e., close to $m_{f_{1}}-m_{\pi} \approx 1147 \mathrm{MeV}$ and a bump in the $K \pi$ spectrum near its threshold. This mechanism obviously cannot be responsible for any significant contribution to the $f_{1}(1285) \rightarrow K \bar{K} \pi$ decay. We are also unable to suggest any special enhancement of the $f_{1}(1285) \rightarrow f_{0}(980) \pi^{0} \rightarrow \pi^{+} \pi^{-} \pi^{0}$ decay due to that mechanism.

If the mass of the $K_{0}^{*}$ resonance is increased (moved away from the $K \pi$ threshold, $m_{K}+m_{\pi} \approx 0.629 \mathrm{GeV}$ ), the disagreement with the data on the $K \bar{K}$ and $K \pi$ spectra gradually decreases. The resonance $K_{0}^{*}(1430)$ with the mass $m_{K_{0}^{*}} \approx 1425 \mathrm{MeV}$ and width $\Gamma_{K_{0}^{*}} \approx 270 \mathrm{MeV}[9]$ can be considered a candidate responsible for the decays $f_{1}(1285) \rightarrow\left(K_{0}^{*} \bar{K}+\bar{K}_{0}^{*} K\right) \rightarrow K \bar{K} \pi$ and $f_{1}(1285) \rightarrow$ $\left(K_{0}^{*} \bar{K}+\bar{K}_{0}^{*} K\right) \rightarrow\left(K^{+} K^{-}+K^{0} \bar{K}^{0}\right) \pi^{0} \rightarrow f_{0}(980) \pi^{0} \rightarrow$ $\pi^{+} \pi^{-} \pi^{0}$. It is then quite natural that the $\pi^{+} \pi^{-}$mass spectrum in the decay $f_{1}(1285) \rightarrow\left(K_{0}^{*} \bar{K}+\bar{K}_{0}^{*} K\right) \rightarrow$ $\left(K^{+} K^{-}+K^{0} \bar{K}^{0}\right) \pi^{0} \rightarrow f_{0}(980) \pi^{0} \rightarrow \pi^{+} \pi^{-} \pi^{0}$ is similar to the $\pi^{+} \pi^{-}$mass spectra in Figs 3 and 9. The calculated ratio of the decay widths corresponding to the mechanism involving the $K_{0}^{*}(1430)$ resonance is [95]

$$
\begin{gathered}
\frac{\Gamma_{f_{1} \rightarrow f_{0} \pi^{0} \rightarrow \pi^{+} \pi^{-} \pi^{0}}}{\Gamma_{f_{1} \rightarrow\left(K_{0}^{*} \bar{K}+\bar{K}_{0}^{*} K\right) \rightarrow K \bar{K} \pi}} \\
=\frac{B R\left(f_{1} \rightarrow f_{0} \pi^{0} \rightarrow \pi^{+} \pi^{-} \pi^{0}\right)}{B R\left(f_{1} \rightarrow\left(K_{0}^{*} \bar{K}+\bar{K}_{0}^{*} K\right) \rightarrow K \bar{K} \pi\right)} \\
=0.271 \times 10^{-2} .
\end{gathered}
$$


Because estimate (74) virtually coincides with (72), the conclusions regarding $B R\left(f_{1} \rightarrow f_{0} \pi^{0} \rightarrow \pi^{+} \pi^{-} \pi^{0}\right)$ that follow Eqn (72) hold in this case as well. Thus, the transition mechanism

$$
\begin{gathered}
f_{1}(1285) \rightarrow\left(K_{0}^{*} \bar{K}+\bar{K}_{0}^{*} K\right) \rightarrow\left(K^{+} K^{-}+K^{0} \bar{K}^{0}\right) \pi^{0} \\
\rightarrow f_{0}(980) \pi^{0} \rightarrow \pi^{+} \pi^{-} \pi^{0}
\end{gathered}
$$

cannot per se explain the experimental data.

Summarizing, we have considered four possible mechanisms of the isospin-violating decay $f_{1}(1285) \rightarrow \pi^{+} \pi^{-} \pi^{0}$. The conclusions we draw from the obtained estimates are as follows. The experimental data can hardly be explained by any of the considered mechanisms. On the other hand, the common feature of these mechanisms is that in each of them the $\pi^{+} \pi^{-}$mass spectrum in the $f_{1}(1285) \rightarrow \pi^{+} \pi^{-} \pi^{0}$ decay is concentrated between the $K^{+} K^{-}$and $K^{0} \bar{K}^{0}$ thresholds due to the $K \bar{K}$-loop character of isotopic symmetry violation. The considered mechanisms are obviously a basis of the isospin violation in the $f_{1}(1285) \rightarrow \pi^{+} \pi^{-} \pi^{0}$ decay; however, the situation requires further elucidation. For example, it is still difficult to answer the question whether the significant probability of the $f_{1}(1285) \rightarrow \pi^{+} \pi^{-} \pi^{0}$ decay can be explained by the joint action of the described mechanisms. Significant experimental efforts are needed to remove uncertainties in the currently available data (for example, it is desirable to measure different modes of the $f_{1}(1285)$ decay concurrently at the same facility and identify the mechanisms of the $f_{1}(1285)$ decay into $\eta \pi \pi$ and $K \bar{K} \pi$ more accurately). Because data on the probability of the $f_{1}(1285) \rightarrow \pi^{+} \pi^{-} \pi^{0}$ decay vary in a broad range [compare (33), (52), and (53)], an improvement in the accuracy of these data is a challenging and important task.

\subsection{Consistency condition}

We use the decay $f_{1}(1285) \rightarrow f_{0}(980) \pi^{0} \rightarrow \pi^{+} \pi^{-} \pi^{0}$ as an example to discuss a more general approach to estimating the contribution from the $K \bar{K}$-loop mechanisms of isotopic invariance violation. We formulate it as a consistency requirement for data on the decays $f_{1}(1285) \rightarrow \pi^{+} \pi^{-} \pi^{0}$ and $f_{1}(1285) \rightarrow K \bar{K} \pi$ based on the concept that isotopic invariance is violated due to the mass difference between $K^{+}$and $K^{0}$ mesons.

We consider the diagrams shown in Figs 10 and 11. If $f_{1}(1285)$ decays into $K \bar{K} \pi$ (this occurs, as was noted above, in approximately $9 \%$ of cases $[9]$ ), then, due to final-state interaction between $K$ and $\bar{K}$ mesons, i.e., transitions $K^{+} K^{-} \rightarrow f_{0}(980) \rightarrow \pi^{+} \pi^{-}$and $K^{0} \bar{K}^{0} \rightarrow$ $f_{0}(980) \rightarrow \pi^{+} \pi^{-}$, the isospin-violating decay $f_{1}(1285) \rightarrow$ $\left(K^{+} K^{-}+K^{0} \bar{K}^{0}\right) \pi^{0} \rightarrow f_{0}(980) \pi^{0} \rightarrow \pi^{+} \pi^{-} \pi^{0}$ is induced (see Fig. 11). Regardless of the specific mechanism of $K \bar{K}$-pair production, this results, as has been shown above, in the emergence in the $\pi^{+} \pi^{-}$mass spectrum of a narrow resonance structure with a width $\approx 2 m_{K^{0}}-$ $2 m_{K^{+}} \approx 8 \mathrm{MeV}$, located in the region of $K \bar{K}$ thresholds. Observing this structure experimentally would be a direct indication of the general $K \bar{K}$-loop nature of the

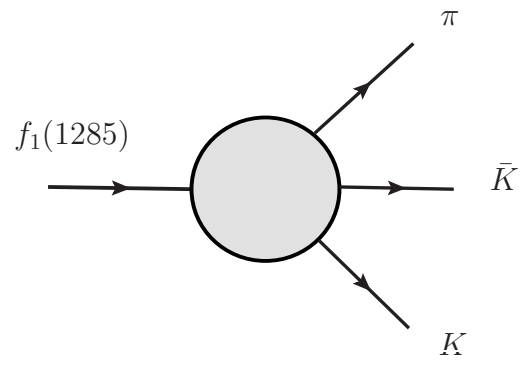

Figure 10: Diagram of the decay $f_{1}(1285) \rightarrow K \bar{K} \pi$.

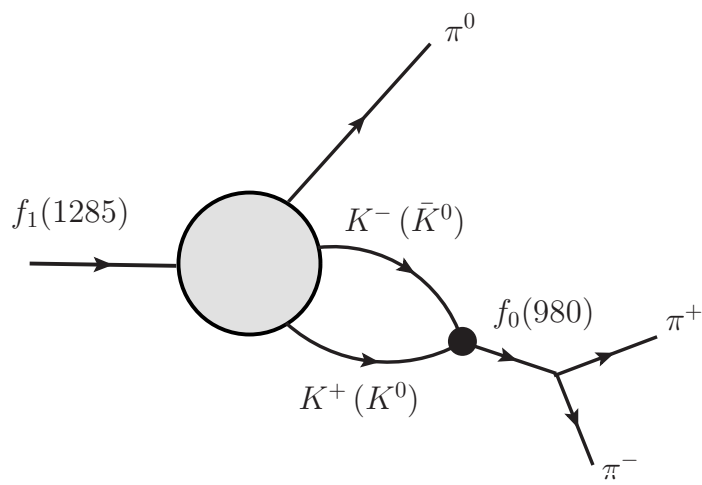

Figure 11: Diagram corresponding to the combined contribution of all possible $K \bar{K}$-loop mechanisms resulting in the decay $f_{1}(1285) \rightarrow\left(K^{+} K^{-}+K^{0} \bar{K}^{0}\right) \pi^{0} \rightarrow f_{0}(980) \pi^{0} \rightarrow$ $\pi^{+} \pi^{-} \pi^{0}$.

mechanisms responsible for isotopic invariance violation.

We expand the isospin-violating amplitude $\mathcal{M}_{f_{1} \rightarrow f_{0} \pi^{0}}(s)$ near the $K \bar{K}$ thresholds in a power series in $\rho_{K \bar{K}}(m)=\sqrt{1-4 m_{K}^{2} / m^{2}}$ :

$$
\begin{aligned}
& \mathcal{M}_{f_{1}(1285) \rightarrow f_{0}(980) \pi^{0}}(m)=g_{f_{0} K^{+} K^{-}}\{A(m) \\
& \times i\left[\rho_{K^{+} K^{-}}(m)-\rho_{K^{0} \bar{K}^{0}}(m)\right]+B(m)\left[\rho_{K^{+} K^{-}}^{2}(m)\right. \\
& \left.\left.-\rho_{K^{0} \bar{K}^{0}}^{2}(m)\right]+O\left[\rho_{K^{+} K^{-}}^{3}(m)-\rho_{K^{0} \bar{K}^{0}}^{3}(m)\right]+\cdots\right\} .
\end{aligned}
$$

With good accuracy,

$$
\begin{aligned}
& \mathcal{M}_{f_{1}(1285) \rightarrow f_{0}(980) \pi^{0}}(m)=g_{f_{0} K^{+} K^{-}} A(m) \\
& \quad \times i\left[\rho_{K^{+} K^{-}}(m)-\rho_{K^{0} \bar{K}^{0}}(m)\right] .
\end{aligned}
$$

The amplitude $A(m)$ contains information about all possible production mechanisms of the $K \bar{K}$ system with the isospin $I=1$ in the $S$-wave in the process $f_{1}(1285) \rightarrow$ $K \bar{K} \pi$.

Data on the decay $f_{1}(1285) \rightarrow f_{0}(980) \pi^{0} \rightarrow \pi^{+} \pi^{-} \pi^{0}$ can be used to extract information about $|A(m)|^{2}$ in the region of $K^{+} K^{-}$and $K^{0} \bar{K}^{0}$ thresholds:

$$
\begin{aligned}
& \frac{d \Gamma_{f_{1}(1285) \rightarrow f_{0}(980) \pi^{0} \rightarrow \pi^{+} \pi^{-} \pi^{0}}(m)}{d m} \\
& =\frac{1}{16 \pi}\left|\mathcal{M}_{f_{1}(1285) \rightarrow f_{0}(980) \pi^{0}}(m)\right|^{2}
\end{aligned}
$$




$$
\times p^{3}(m) \frac{2 m^{2} \Gamma_{f_{0} \rightarrow \pi^{+} \pi^{-}}(m)}{\pi\left|D_{f_{0}}(m)\right|^{2}},
$$

where

$$
p(m)=\frac{\left[m_{f_{1}}^{4}-2 m_{f_{1}}^{2}\left(m^{2}+m_{\pi}^{2}\right)+\left(m^{2}-m_{\pi}^{2}\right)^{2}\right]^{1 / 2}}{2 m_{f_{1}}} .
$$

Information about $|A(m)|^{2}$ at $m>2 m_{K}$ can also be obtained from data on the $K \bar{K}$ mass spectra measured in the $f_{1}(1285) \rightarrow K \bar{K} \pi$ decay. Unfortunately, data on those spectra are still rather scant 149 151]. But in the case of large statistics and good resolution in the invariant mass of the $K \bar{K}$ system, a straightforward scheme for obtaining data about $|A(m)|^{2}$ at $m$ above the $K^{+} K^{-}$, $K^{0} \bar{K}^{0}$, or $K^{ \pm} K_{S}^{0}$ threshold could be as follows.

The $K \bar{K}$ system in the $f_{1}(1285) \rightarrow K \bar{K} \pi$ decay (see Fig. 10) is generated predominantly in the $S$-wave due to the very restricted phase volume available for it $\left(2 m_{K}<\right.$ $\left.m<2 m_{K}+150 \mathrm{MeV}\right)$. Then, for example, the $K^{+} K^{-}$ mass spectrum in the decay $f_{1}(1285) \rightarrow K^{+} K^{-} \pi^{0}$ can be represented in the form

$$
\begin{aligned}
& \frac{d \Gamma_{f_{1}(1285) \rightarrow K^{+} K^{-} \pi^{0}}(m)}{d m} \\
= & \frac{2 m}{\pi} \rho_{K^{+} K^{-}}(m) p^{3}(m)|A(m)|^{2} .
\end{aligned}
$$

By approximating data on $d \Gamma_{f_{1} \rightarrow K^{+} K^{-} \pi^{0}}(m) / d m$, we can determine the function $|A(m)|^{2}$ and use its value $\left|A\left(2 m_{K^{+}}\right)\right|^{2}$ at the $K^{+} K^{-}$threshold (which certainly corresponds to the contribution of the $S$-wave alone) to derive the following approximate estimate for $\Gamma_{f_{1} \rightarrow f_{0} \pi^{0} \rightarrow \pi^{+} \pi^{-} \pi^{0}}$ [95]:

$$
\begin{gathered}
\Gamma_{f_{1} \rightarrow f_{0} \pi^{0} \rightarrow \pi^{+} \pi^{-} \pi^{0}}=\int \frac{d \Gamma_{f_{1} \rightarrow f_{0} \pi^{0} \rightarrow \pi^{+} \pi^{-} \pi^{0}}(m)}{d m} d m \\
=\left|A\left(2 m_{K^{+}}\right)\right|^{2} \int\left|\rho_{K^{+} K^{-}}(m)-\rho_{K^{0} \bar{K}^{0}}(m)\right|^{2} \\
\times p^{3}(m) \frac{g_{f_{0} K^{+} K^{-}}^{2}}{16 \pi} \frac{2 m^{2} \Gamma_{f_{0} \rightarrow \pi^{+} \pi^{-}}(m)}{\pi\left|D_{f_{0}}(m)\right|^{2}} d m \\
\approx\left|A\left(2 m_{K^{+}}\right)\right|^{2} 2.59 \times 10^{-6} \mathrm{GeV}^{5} .
\end{gathered}
$$

A comparison of this estimate with data on $f_{1}(1285) \rightarrow$ $\pi^{+} \pi^{-} \pi^{0}$ decay enables checking their consistency with the data on the $f_{1}(1285) \rightarrow K \bar{K} \pi$ decay and the concept of isotopic invariance violation due to the mass difference between $K^{+}$and $K^{0}$ mesons.

The considered approach can also be used to obtain estimates for other decays of the same type.

If the isospin-violating amplitude contains logarithmic singularities near the $K \bar{K}$ thresholds in the physical region of kinematic variables (as is the case, for example, in the decay $\eta(1405) \rightarrow\left(K^{*} \bar{K}+\bar{K}^{*} K\right) \rightarrow\left(K^{+} K^{-}+\right.$ $\left.K^{0} \bar{K}^{0}\right) \pi^{0} \rightarrow f_{0}(980) \pi^{0} \rightarrow \pi^{+} \pi^{-} \pi^{0}$, which we discuss below), its structure at $m \approx 2 m_{K}$ is more involved than expression (75). The amplitude near the singularities cannot be straightforwardly expanded in $\rho_{K \bar{K}}(m)$, and a simple consistency condition such as that in (80) cannot be obtained. However, the $\pi^{+} \pi^{-}$mass spectrum in the decay $\eta(1405) \rightarrow \pi^{+} \pi^{-} \pi^{0}$ is also concentrated in the region between the $K^{+} K^{-}$and $K^{0} \bar{K}^{0}$ thresholds, and therefore effectively corresponds to the shape characteristic of the $K \bar{K}$-loop mechanism of isotopic invariance violation [135].

5.3. $K \bar{K}$-loop mechanism of isotopic invariance violation in the decay $\eta(1405) \rightarrow f_{0}(980) \pi^{0} \rightarrow 3 \pi$ and the role of anomalous Landau thresholds

In 2012, the BESIII collaboration measured the decays $J / \psi \rightarrow \gamma \pi^{+} \pi^{-} \pi^{0}$ and $J / \psi \rightarrow \gamma \pi^{0} \pi^{0} \pi^{0}$ and found a resonance peak about $50 \mathrm{MeV}$ wide in the three-pion mass spectra around $1.4 \mathrm{GeV}$ [125]. The corresponding $\pi^{+} \pi^{-}$and $\pi^{0} \pi^{0}$ mass spectra in the $990 \mathrm{MeV}$ range (i.e., in the vicinity of $K^{+} K^{-}$and $K^{0} \bar{K}^{0}$ thresholds) proved to contain a narrow structure whose width is about 10 $\mathrm{MeV}$ [125]. Thus, the experiment was the first observation (with a statistical confidence of $10 \mathrm{~s}$ ) of the isospinviolating decay $J / \psi \rightarrow \gamma \eta(1405) \rightarrow \gamma f_{0}(980) \pi^{0}$ followed by the decay $f_{0}(980) \rightarrow \pi^{+} \pi^{-}, \pi^{0} \pi^{0}$. According to the data in [125],

$$
\begin{aligned}
B R(J / \psi & \left.\rightarrow \gamma \eta(1405) \rightarrow \gamma f_{0}(980) \pi^{0} \rightarrow \gamma \pi^{+} \pi^{-} \pi^{0}\right) \\
& =(1.50 \pm 0.11 \pm 0.11) \times 10^{-5} .
\end{aligned}
$$

Using the PDG data, the BESIII collaboration [125] also obtained the ratio

$$
\begin{aligned}
& \frac{B R\left(\eta(1405) \rightarrow f_{0}(980) \pi^{0} \rightarrow \pi^{+} \pi^{-} \pi^{0}\right)}{B R\left(\eta(1405) \rightarrow a_{0}^{0}(980) \pi^{0} \rightarrow \eta \pi^{0} \pi^{0}\right)} \\
& =(17.9 \pm 4.2) \%
\end{aligned}
$$

whose value virtually precludes an explanation of the discovered effect of isotopic invariance violation by the $a_{0}^{0}(980)-f_{0}(980)$ mixing. At the same time, the narrow resonance-like structure discovered in $\pi^{+} \pi^{-}$and $\pi^{0} \pi^{0}$ mass spectra in the decay $\eta(1405) \rightarrow \pi^{+} \pi^{-} \pi^{0}, \pi^{0} \pi^{0} \pi^{0}$ in the vicinity of the $K^{+} K^{-}$and $K^{0} \bar{K}^{0}$ thresholds suggests that the mechanism responsible for the production of the $f_{0}(980)$ resonance in the decay $\eta(1405) \rightarrow f_{0}(980) \pi^{0} \rightarrow 3 \pi$ is similar to that of $a_{0}^{0}(980)-f_{0}(980)$ mixing, i.e., due to the $K \bar{K}$-loop transition $\eta(1405) \rightarrow\left(K^{+} K^{-}+\right.$ $\left.K^{0} \bar{K}^{0}\right) \pi^{0} \rightarrow f_{0}(980) \pi^{0} \rightarrow 3 \pi$, whose amplitude does not vanish owing to the mass difference between $K^{+}$and $K^{0}$ mesons and has a significant magnitude in the narrow region between the $K^{+} K^{-}$and $K^{0} \bar{K}^{0}$ thresholds.

Comparing the result in (81) obtained by BESIII with the PDG data [9] for the dominant decay channel $J / \psi \rightarrow$ $\gamma \eta(1405 / 1475) \rightarrow \gamma K \bar{K} \pi$,

$$
\begin{aligned}
B R(J / \psi & \rightarrow \gamma \eta(1405 / 1475) \rightarrow \gamma K \bar{K} \pi) \\
& =(2.8 \pm 0.6) \cdot 10^{-3},
\end{aligned}
$$

yields

$$
\begin{gathered}
\left.\frac{B R\left(J / \psi \rightarrow \gamma \eta(1405) \rightarrow \gamma f_{0}(980) \pi^{0} \rightarrow \gamma \pi^{+} \pi^{-} \pi^{0}\right)}{B R(J / \psi} \rightarrow \gamma \eta(1405 / 1475) \rightarrow \gamma K \bar{K} \pi\right) \\
=(0.53 \pm 0.13) \% .
\end{gathered}
$$




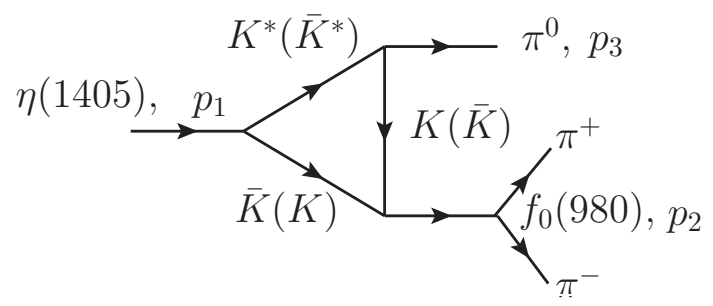

Figure 12: Diagram of the decay $\eta(1405) \rightarrow\left(K^{*} \bar{K}+\bar{K}^{*} K\right) \rightarrow$ $K \bar{K} \pi^{0} \rightarrow f_{0}(980) \pi^{0} \rightarrow \pi^{+} \pi^{-} \pi^{0}$. All intermediate particles in the triangle loop of this diagram in the region of $\eta(1405)$ resonance may be on the mass shell. As a result, a logarithmic singularity emerges in the imaginary part of the trianglediagram amplitude in the case of a hypothetical stable $K^{*}$ meson [135, 152 154]. $p_{1}, p_{2}$, and $p_{3}$ denote the 4-momenta of the particle in the reaction; $p_{1}^{2}=s_{1}$ is the invariant mass squared of $\eta(1405)$-resonance or the final $\pi^{+} \pi^{-} \pi^{0}$ system; $p_{2}^{2}=s_{2}$ is the invariant mass squared of $f_{0}(980)$ or the final $\pi^{+} \pi^{-}$system; and $p_{3}^{2}=m_{\pi^{0}}^{2}$.

The value of the last ratio is also indicative of a very large violation of isospin invariance in the decay $\eta(1405) \rightarrow f_{0}(980) \pi^{0}$.

Below, we consider the theoretical possibility of explaining the strong violation of isotopic invariance in the decay $\eta(1405) \rightarrow f_{0}(980) \pi^{0} \rightarrow \pi^{+} \pi^{-} \pi^{0}$ by anomalous Landau thresholds (or logarithmic triangular singularities) that are present in the amplitude of the transition $\eta(1405) \rightarrow\left(K^{*} \bar{K}+\bar{K}^{*} K\right) \rightarrow K \bar{K} \pi^{0} \rightarrow f_{0}(980) \pi^{0} \rightarrow$ $\pi^{+} \pi^{-} \pi^{0}$ (Fig. 12) near the $K \bar{K}$ thresholds. An attempt to explain the decay $\eta(1405) \rightarrow f_{0}(980) \pi^{0} \rightarrow \pi^{+} \pi^{-} \pi^{0}$ invoking that mechanism was made in [126 128]. Shortly after that, we noted that in the calculations, the vector $K^{*}$ meson $K^{*}(892)$ was considered a stable particle in the intermediate state; we showed in [135] that taking its finite width $\Gamma_{K^{*}} \approx \Gamma_{K^{*} \rightarrow K \pi} \approx 50 \mathrm{MeV}$ into account smoothes the logarithmic singularities in the amplitude and reduces the calculated probability of the decay $\eta(1405) \rightarrow f_{0}(980) \pi^{0} \rightarrow \pi^{+} \pi^{-} \pi^{0}$ by a factor of 6 to 8 compared to that with $\Gamma_{K^{*}}=0$. Also assuming the dominance of the decay $\eta(1405) \rightarrow\left(K^{*} \bar{K}+\bar{K}^{*} K\right) \rightarrow K \bar{K} \pi^{0}$, we obtained the estimate [135]

$$
\begin{gathered}
B R\left(J / \psi \rightarrow \gamma \eta(1405) \rightarrow \gamma f_{0}(980) \pi^{0} \rightarrow \gamma \pi^{+} \pi^{-} \pi^{0}\right) \\
\approx 1.12 \cdot 10^{-5}
\end{gathered}
$$

which agrees with the BESIII data 125] quoted in (81) reasonably well.

To show the effect of the $K^{*}$-meson width on the calculation of the isospin-violating diagram displayed in Fig. 12 in the most transparent way, we ignore the spin effects, which only make the intermediate calculations significantly more complicated [135], having actually no effect whatsoever on the final result [155] (i.e., we treat $K^{*}$ as a spinless particle in what follows). ${ }^{6}$

For the amplitude of the triangle loop in Fig. 12, we introduce the notation

$$
T=2 \frac{g_{1} g_{2} g_{3}}{16 \pi}\left[F_{+}\left(s_{1}, s_{2}\right)-F_{0}\left(s_{1}, s_{2}\right)\right],
$$

where $g_{1}, g_{2}$, and $g_{3}$ are the coupling constants at the vertices (which are assumed to be the same for charged and neutral channels), the amplitudes $F_{+}\left(s_{1}, s_{2}\right)$ and $F_{0}\left(s_{1}, s_{2}\right)$ describe the respective contributions of the charged and neutral intermediate states, and the factor 2 appears because there are two such contributions. We relate the isospin violation in the considered diagram only to the difference in mass between charged and neutral $K$ mesons and set $m_{K^{*+}}=m_{K^{* 0}}=0.8955 \mathrm{GeV}$. The amplitude $F_{+} \equiv F_{+}\left(s_{1}, s_{2}\right)$ is given by

$$
F_{+}=\frac{i}{\pi^{3}} \int \frac{d^{4} k}{D_{1} D_{2} D_{3}}
$$

where $D_{1}=\left(k^{2}-m_{K^{*+}}^{2}+i \varepsilon\right), D_{2}=\left(\left(p_{1}-k\right)^{2}-m_{K^{-}}^{2}+i \varepsilon\right)$, and $D_{3}=\left(\left(k-p_{3}\right)^{2}-m_{K^{+}}^{2}+i \varepsilon\right)$ are inverse propagators of loop particles. The imaginary part of $F_{+}$in the region $s_{1} \geq\left(m_{K^{*+}}+m_{K^{+}}\right)^{2}$ and $s_{2} \geq 4 m_{K^{+}}^{2}$ consists of the imaginary part due to the discontinuity on the $K^{*+} K^{-}$ cut in the variable $s_{1}$ and the imaginary part due to the discontinuity on the $K^{+} K^{-}$cut in the variable $s_{2}$ :

$$
\begin{gathered}
\operatorname{Im} F_{+}=\operatorname{Im} F_{+}^{\left(K^{*+} K^{-}\right)}+\operatorname{Im} F_{+}^{\left(K^{+} K^{-}\right)}, \\
\operatorname{Im} F_{+}^{\left(K^{*+} K^{-}\right)}=\frac{1}{\sqrt{\Delta}} \ln \left[\frac{\alpha_{+}+\sqrt{\Delta \delta_{+}}}{\alpha_{+}-\sqrt{\Delta \delta_{+}}}\right], \\
\operatorname{Im} F_{+}^{\left(K^{+} K^{-}\right)}=\frac{1}{\sqrt{\Delta}} \ln \left[\frac{\alpha_{+}^{\prime}+\sqrt{\Delta \delta_{+}^{\prime}}}{\alpha_{+}^{\prime}-\sqrt{\Delta \delta_{+}^{\prime}}}\right],
\end{gathered}
$$

where

$$
\begin{gathered}
\Delta=s_{1}^{2}-2 s_{1}\left(s_{2}+m_{\pi^{0}}^{2}\right)+\left(s_{2}-m_{\pi^{0}}^{2}\right)^{2} \\
\alpha_{+}=s_{1}^{2}-s_{1}\left(s_{2}+m_{\pi^{0}}^{2}+m_{K^{*+}}^{2}-m_{K^{+}}^{2}\right) \\
+\left(s_{2}-m_{\pi^{0}}^{2}\right)\left(m_{K^{+}}^{2}-m_{K^{*+}}^{2}\right) \\
\delta_{+}=s_{1}^{2}-2 s_{1}\left(m_{K^{*+}}^{2}+m_{K^{+}}^{2}\right)+\left(m_{K^{*+}}^{2}-m_{K^{+}}^{2}\right)^{2} \\
\alpha_{+}^{\prime}=s_{2}\left(s_{2}-s_{1}-m_{\pi^{0}}^{2}-2 m_{K^{+}}^{2}+2 m_{K^{*+}}^{2}\right) \\
\delta_{+}^{\prime}=s_{2}\left(s_{2}-4 m_{K^{+}}^{2}\right) .
\end{gathered}
$$

\footnotetext{
${ }^{6}$ We note that convergence or divergence of both the triangle diagram and the $K \bar{K}$ loops in the case of the $a_{0}^{0}(980) \rightarrow$ $\left(K^{+} K^{-}+K^{0} \bar{K}^{0}\right) \rightarrow f_{0}(980)$ transition does not have any relation to the effect of isospin violation under consideration. The total of the subtraction constants for the contributions from charged and neutral intermediate states to the dispersion relation for the isospin-violating amplitude has a natural order of smallness $\sim\left(m_{K^{0}}-m_{K^{+}}\right)$and cannot be responsible for the enhanced symmetry violation in a narrow region near the $K^{+} K^{-}$ and $K^{0} \bar{K}^{0}$ thresholds.
} 


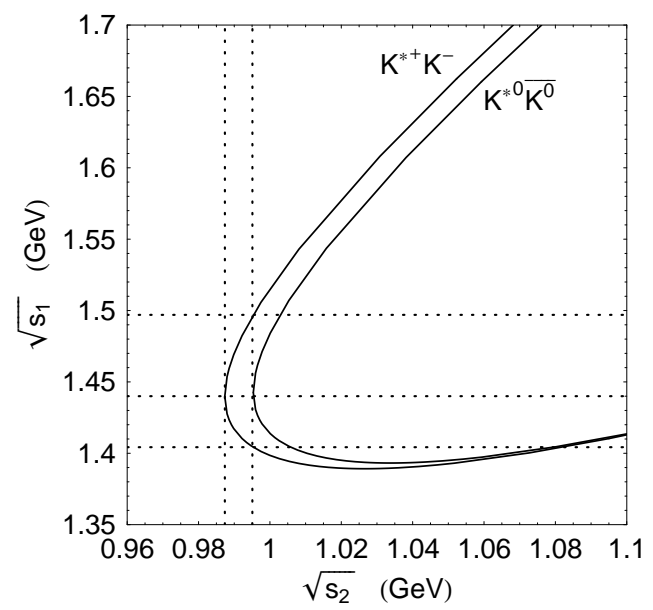

Figure 13: Solid curves in the $\left(\sqrt{s_{2}}, \sqrt{s_{1}}\right)$ plane show the location of the logarithmic singularity in the imaginary part of the triangle diagram (see Fig. 12) that corresponds to the contributions of the $K^{*+} K^{-}$and $K^{* 0} \bar{K}^{0}$ intermediate states. The dotted vertical lines show the $K^{+} K^{-}$and $K^{0} \bar{K}^{0}$ thresholds in the variable $\sqrt{s_{2}}$ (i.e., its value $2 m_{K^{+}}=0.987354 \mathrm{GeV}$ and $2 m_{K^{0}}=0.995344 \mathrm{GeV}$ ). The dotted horizontal lines show the values of the variable $\sqrt{s_{1}} 1.404,1.440$, and $1.497 \mathrm{GeV}$. For $1.404<\sqrt{s_{1}}<1.497 \mathrm{GeV}$, the logarithmic singularity is located in the case of the $K^{*+} K^{-}$intermediate state at a value of $\sqrt{s_{2}}$ between the $K^{+} K^{-}$and $K^{0} \bar{K}^{0}$ thresholds, while in the case of the $K^{* 0} \bar{K}^{0}$ intermediate state, its distance from the $K^{0} \bar{K}^{0}$ thresholds is no more than $6 \mathrm{MeV}$. The singularities touch the $K \bar{K}$ thresholds at approximately $\sqrt{s_{1}}=1.440$ $\mathrm{GeV}$.

Replacing the index + of the functions in (87)-(95) with the index 0 and masses of charged intermediate particles with masses of their neutral counterparts, we obtain everything necessary for describing the amplitude $F_{0} \equiv F_{0}\left(s_{1}, s_{2}\right)$.

A specific feature of the considered case is that all intermediate particles in the triangle diagram in Fig. 12 can be on the mass shell in the region of the $\eta(1405)$ resonance. This situation occurs for the values of the kinematic variables $s_{1}$ and $s_{2}$ related to each other as

$$
\alpha_{+, 0}= \pm \sqrt{\Delta \delta_{+, 0}}
$$

or equivalently

$$
\alpha_{+, 0}^{\prime}= \pm \sqrt{\Delta \delta_{+, 0}^{\prime}}
$$

Therefore, in the hypothetical case of a stable $K^{*}$ meson, the imaginary part of this triangle diagram contains a logarithmic singularity [135, 152 154]. Figure 13 shows the location of the logarithmic singularities due to the contributions of the $K^{*+} K^{-}$and $K^{* 0} \bar{K}^{0}$ intermediate states in the $\left(\sqrt{s_{2}}, \sqrt{s_{1}}\right)$ plane. It can be seen that they come very close to the $K \bar{K}$ thresholds (whose location is shown in this figure and in Figs 14-19 with vertical dotted lines) in the region of the $\eta(1405)$ resonance. For

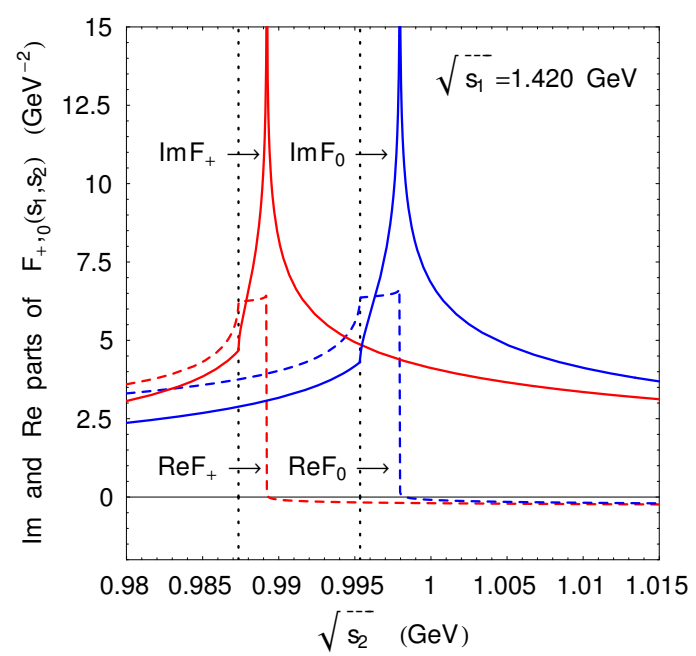

Figure 14: Solid (dashed) curves show the imaginary (real) part of the amplitude $F_{+}\left(s_{1}, s_{2}\right)$ for the charged intermediate state and the amplitude $F_{0}\left(s_{1}, s_{2}\right)$ for the neutral intermediate state in the triangle loop calculated for the hypothetical case of a stable intermediate $K^{*}$ meson.

example, if $\sqrt{s_{1}}=1.420 \mathrm{GeV}$, the singularities in the contributions of the $K^{*+} K^{-}$and $K^{* 0} \bar{K}^{0}$ intermediate states manifest themselves at the respective values $\sqrt{s_{2}} \approx 0.989$ and $0.998 \mathrm{GeV}$ of the $\pi^{+} \pi^{-}$system invariant mass (see Fig. 13). Figure 14 shows a typical behavior of the imaginary and real parts of the amplitudes $F_{+}\left(s_{1}, s_{2}\right)$ and $F_{0}\left(s_{1}, s_{2}\right)$ as functions of $\sqrt{s_{2}}$ in the vicinity of the $K \bar{K}$ thresholds and of $\sqrt{s_{1}}$ in the vicinity of the $\eta(1405)$ resonance, namely, at $\sqrt{s_{1}}=1.420 \mathrm{GeV}$. This behavior is characterized by singularities in $\operatorname{Im} F_{+, 0}\left(s_{1}, s_{2}\right)$ and discontinuities in $\operatorname{Re} F_{+, 0}\left(s_{1}, s_{2}\right)$.

Because the singularities due to the charged and neutral intermediate states, which are located in different places, do not cancel each other, the mechanism under consideration can apparently result in a catastrophic violation of isotopic symmetry in the decay $\eta(1405) \rightarrow$ $\pi^{+} \pi^{-} \pi^{0}$, as illustrated by Fig. 15 .

Such a 'singular' scenario cannot occur in reality. Taking the finite width of the $K^{*}$ resonance into account (i.e., averaging the amplitude over a resonance Breit-Wigner distribution in accordance with the Källén-Lehmann spectral representation for the propagator of an unstable $K^{*}$ meson [152 154]) 'smears out' the logarithmic singularities in the amplitude and in this way enhances the mutual compensation of the contributions of the $\left(K^{*+} K^{-}+K^{*-} K^{+}\right)$and $\left(K^{* 0} \bar{K}^{0}+\bar{K}^{* 0} K^{0}\right)$ intermediate states. This results in a significant decrease in the calculated width of the $\eta(1405) \rightarrow \pi^{+} \pi^{-} \pi^{0}$ decay compared to the case $\Gamma_{K^{*} \rightarrow K \pi}=0$ and in localization of the main effect of isospin violation in the $\pi^{+} \pi^{-}$invariant mass region between the $K \bar{K}$ thresholds.

As stated above, we write the propagator of an unstable $K^{*}$ meson in the form of a spectral Källén-Lehmann 


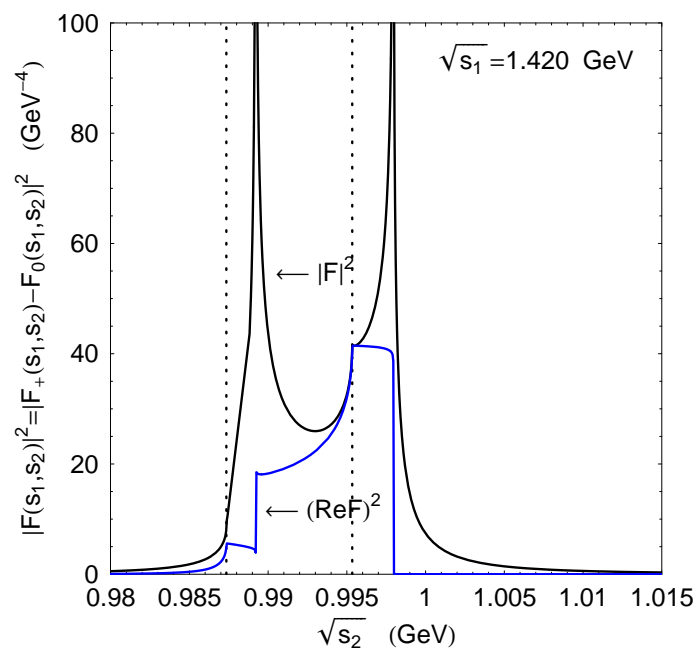

Figure 15: Absolute value squared and the real part squared of the triangle- loop isospin-violating amplitude $F\left(s_{1}, s_{2}\right) \equiv$ $F_{+}\left(s_{1}, s_{2}\right)-F_{0}\left(s_{1}, s_{2}\right)$ for a hypothetical stable intermediate $K^{*}$ meson. The integral contributions from the imaginary and real parts of the amplitude are approximately the same here.

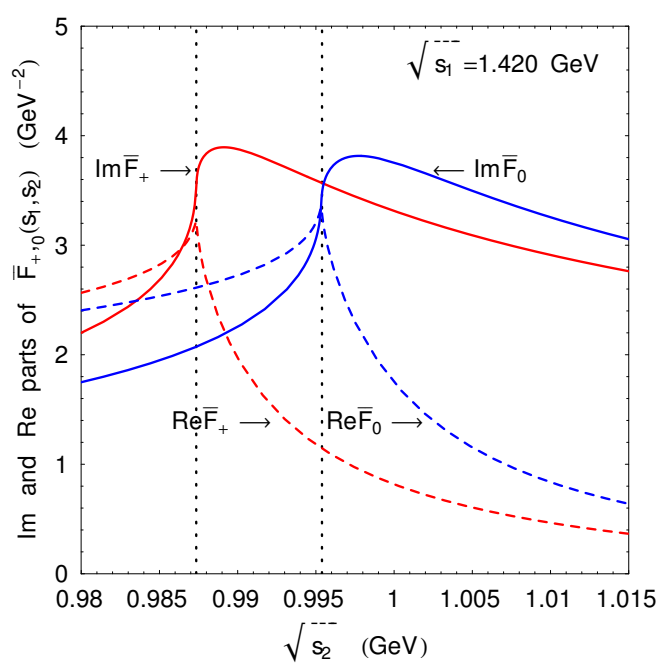

Figure 16: Solid (dashed) curves show the imaginary (real) parts of the amplitude $\bar{F}_{+}\left(s_{1}, s_{2}\right)$ for the charged intermediate state and the amplitude $\bar{F}_{0}\left(s_{1}, s_{2}\right)$ for the neutral intermediate state in the triangle loop calculated taking the instability of the intermediate $K^{*}$ meson into consideration.

representation [152-154]

$$
\frac{1}{m_{K^{*}}^{2}-k^{2}-i m_{K^{*}} \Gamma_{K^{*}}} \rightarrow \int_{\left(m_{K}+m_{\pi}\right)^{2}}^{\infty} d m^{2} \frac{\rho\left(m^{2}\right)}{m^{2}-k^{2}-i \varepsilon}
$$

and approximate $\rho\left(m^{2}\right)$ as

$$
\rho\left(m^{2}\right)=\frac{1}{\pi} \frac{m_{K^{*}} \Gamma_{K^{*}}}{\left(m^{2}-m_{K^{*}}^{2}\right)^{2}+\left(m_{K^{*}} \Gamma_{K^{*}}\right)^{2}} .
$$
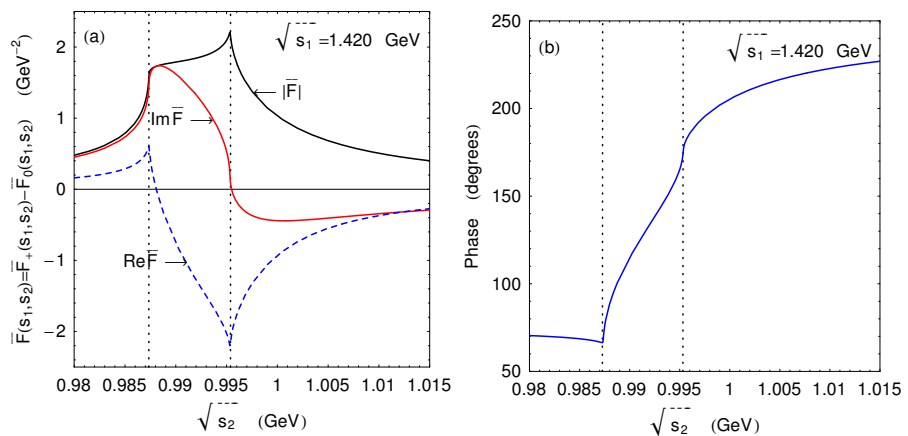

Figure 17: (a) Absolute value, imaginary and real parts of the triangle-loop amplitude $\bar{F}\left(s_{1}, s_{2}\right)=\bar{F}_{+}\left(s_{1}, s_{2}\right)-\bar{F}_{0}\left(s_{1}, s_{2}\right)$ calculated taking the instability of the intermediate $K^{*}$ meson into account. (b) Phase of the amplitude $\bar{F}\left(s_{1}, s_{2}\right)$.

Next, in the formulas for the amplitude $F_{+, 0}\left(s_{1}, s_{2}\right)$, we replace the $K^{*}$-meson mass squared $m_{K^{*}}^{2}$ with the variable mass squared $m^{2}$ and define the amplitudes weighted with the spectral density $\rho\left(m^{2}\right)$ [152 154$]$,

$\bar{F}_{+, 0}\left(s_{1}, s_{2}\right)=\int_{\left(m_{K}+m_{\pi}\right)^{2}}^{\infty} \rho\left(m^{2}\right) F_{+, 0}\left(s_{1}, s_{2} ; m^{2}\right) d m^{2} \cdot(100)$

Figure 16 illustrates the behavior of the imaginary and real parts of the weighted amplitudes $\bar{F}_{+}\left(s_{1}, s_{2}\right)$ and $\bar{F}_{0}\left(s_{1}, s_{2}\right)$ as a function of $\sqrt{s_{2}}$ in the region of $K \bar{K}$ thresholds at $\sqrt{s_{1}}=1.420 \mathrm{GeV}$. A comparison of this figure with Fig. 14 shows that the singular behavior of $F_{+}\left(s_{1}, s_{2}\right)$ and $F_{0}\left(s_{1}, s_{2}\right)$ almost completely disappears if the $K^{*}$ meson instability is taken into account.

The absolute value, the imaginary and real parts, and the phase of the amplitude of the triangle loop $\bar{F}\left(s_{1}, s_{2}\right) \equiv \bar{F}_{+}\left(s_{1}, s_{2}\right)-\bar{F}_{0}\left(s_{1}, s_{2}\right)$ that violates isotopic invariance calculated with the instability of the intermediate $K^{*}$ meson taken into account are displayed in Fig. 17. We can see that all the characteristic features of the amplitude $\bar{F}\left(s_{1}, s_{2}\right)$ are linked to the $K \bar{K}$ thresholds, and the behavior of its absolute value and phase are essentially similar to those of the $a_{0}^{0}(980)-f_{0}(980)$ mixing amplitude $\Pi_{a_{0}^{0} f_{0}}(m)$ shown in Fig. 2.

Shown in Fig. 18 is the absolute value squared of the amplitude $\bar{F}\left(s_{1}, s_{2}\right)=\bar{F}_{+}\left(s_{1}, s_{2}\right)-\bar{F}_{0}\left(s_{1}, s_{2}\right)$, which was obtained taking the instability of the intermediate $K^{*}$ meson into account. It should be compared with its ana$\log$ for $\Gamma_{K^{*}}=0$ shown in Fig. 15. It is noteworthy that the areas below the corresponding curves differ by about an order of magnitude. This is the effect of the finite width $\Gamma_{K^{*}}=50 \mathrm{MeV}$.

A similar situation occurs for all values of $\sqrt{s_{1}}$ in the region of the $\eta(1405)$ resonance. Figure 19 shows the overall view of the $\pi^{+} \pi^{-}$mass spectrum in the decay $\eta(1405) \rightarrow \pi^{+} \pi^{-} \pi^{0}$ calculated for the nominal mass of 


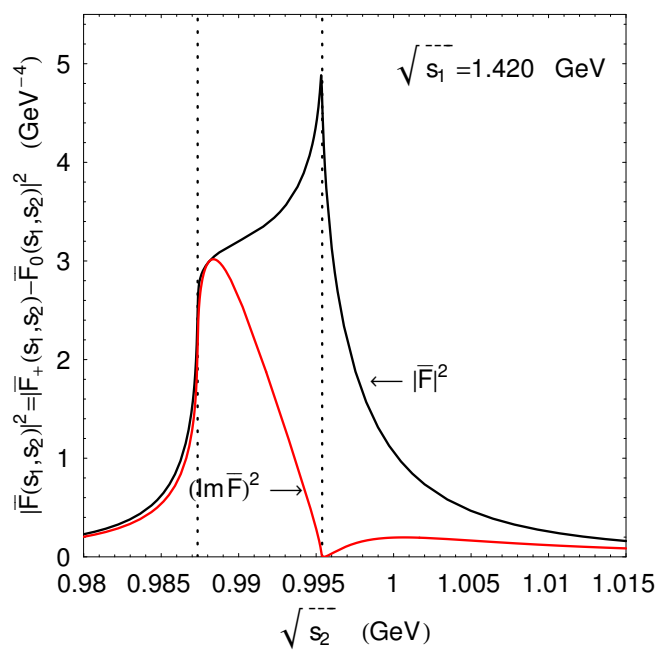

Figure 18: Absolute value squared and the imaginary part squared of the triangle-loop amplitude $\bar{F}\left(s_{1}, s_{2}\right)=$ $\bar{F}_{+}\left(s_{1}, s_{2}\right)-\bar{F}_{0}\left(s_{1}, s_{2}\right)$ calculated taking the instability of the intermediate $K^{*}$ meson into consideration. The plots should be compared with Fig. 15.

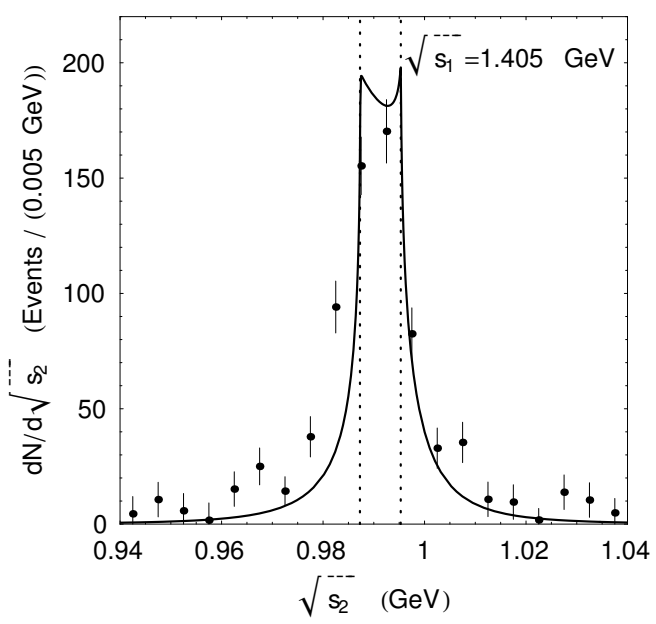

Figure 19: Illustration of the $\pi^{+} \pi^{-}$mass spectrum shape in the decay $\eta(1405) \rightarrow \pi^{+} \pi^{-} \pi^{0}$ plotted using Eqn (101), which corresponds to the contribution of the diagram in Fig. 12. The dots with error bars represent the first BESIII data for this decay [125].

$\eta(1405)$, i.e., at $\sqrt{s_{1}}=1.405 \mathrm{GeV}$, using the formula

$$
\begin{aligned}
\frac{d N}{d \sqrt{s_{2}}}=C & \sqrt{\frac{\Delta}{s_{1}}}\left|\bar{F}_{+}\left(s_{1}, s_{2}\right)-\bar{F}_{0}\left(s_{1}, s_{2}\right)\right|^{2} \\
& \times \frac{s_{2} \Gamma_{f_{0} \rightarrow \pi^{+} \pi^{-}}\left(\sqrt{s_{2}}\right)}{\pi\left|D_{f_{0}}\left(\sqrt{s_{2}}\right)\right|^{2}},
\end{aligned}
$$

where $C$ is a normalization constant.

In conclusion, we consider the high-level hierarchy of isotopic-symmetry violations induced by the mass differ- ence of $K^{+}$and $K^{0}$ mesons discussed above.

The baseline isotopic symmetry violation in the process amplitude is of the order of

$$
\simeq \frac{m_{K^{0}}-m_{K^{+}}}{m_{K^{0}}} \approx \frac{1}{126} .
$$

Symmetry violation in the process amplitude in the region between the $K^{+} K^{-}$and $K^{0} \bar{K}^{0}$ thresholds owing to any mechanism of production of $K \bar{K}$ pairs with a definite isospin in the $S$-wave without anomalous Landau thresholds, in particular, as a result of the $a_{0}^{0}(980)-f_{0}(980)$ mixing, is of the order of

$$
\simeq \sqrt{\frac{2\left(m_{K^{0}}-m_{K^{+}}\right)}{m_{K^{0}}}} \approx 0.127 .
$$

Symmetry violation in the amplitude of the decay $\eta(1405) \rightarrow f_{0}(980) \pi^{0} \rightarrow \pi^{+} \pi^{-} \pi^{0}$ due to logarithmic triangular singularities in the contributions of $\left(K^{*} \bar{K}+\right.$ $\bar{K}^{*} K$ ) intermediate states in the $\sqrt{s_{2}}$ region between the $K^{0} \bar{K}^{0}$ and $K^{+} K^{-}$thresholds [135] is of the order of

$$
\simeq|\ln | \frac{\Gamma_{K^{*}} / 2}{\sqrt{m_{K^{0}}^{2}-m_{K^{+}}^{2}+\Gamma_{K^{*}}^{2} / 4}}|| \approx 1
$$

(this estimate of the uncompensated part of the contributions of charged and neutral intermediate states in the triangle diagram, which is in agreement with Fig. 17a, can be obtained, for example, from Eqn (90) if $m_{K^{*}}^{2}$ at the singularity point is replaced with $\left.m_{K^{*}}^{2}-i m_{K^{*}} \Gamma_{K^{*}}\right)$.

In all of the cases of anomalous isotopic symmetry violation, the phase of the symmetry-violating amplitude varies in the region between the $K^{+} K^{-}$and $K^{0} \bar{K}^{0}$ thresholds by approximately $90^{\circ}$.

\section{Manifestation of $a_{0}^{0}(980)-f_{0}(980)$ mix- ing in decays of charmed mesons}

Studies of the spectroscopy of light resonances (in particular, $a_{0}(980)$ and $f_{0}(980)$ ) in weak hadronic decays of $D$ and $D_{s}$ mesons is one of the main areas of experimental activities in charmed particle physics at the CERN LHCb detector [84, 85]. We show below that observation of the effects related to $a_{0}^{0}(980)-f_{0}(980)$ mixing in the decays $D_{c}^{+} \rightarrow \eta \pi^{0} \pi^{+}$[140], $D^{0} \rightarrow K_{S}^{0} \pi^{+} \pi^{-}$ and $D^{0} \rightarrow K_{S}^{0} \eta \pi^{0}$ [141] can shed additional light on the mechanisms of $f_{0}(980)$ and $a_{0}(980)$ production in threeparticle hadronic decays of the $D_{s}^{+}$and $D^{0}$ mesons (the nature of these mechanisms is far from being well understood at the current stage of research.) The huge statistics that can be collected in LHCb experiments makes successful detection of the $a_{0}^{0}(980)-f_{0}(980)$ mixing effects quite a feasible task.

\subsection{Decay $D_{s}^{+} \rightarrow \eta \pi^{0} \pi^{+}$}

Figure 20 displays data on the $S$-wave spectrum of the $K^{+} K^{-}$system mass in the decay $D_{s}^{+} \rightarrow K^{+} K^{-} \pi^{+}$ that were obtained by the BaBar collaboration [156]. 


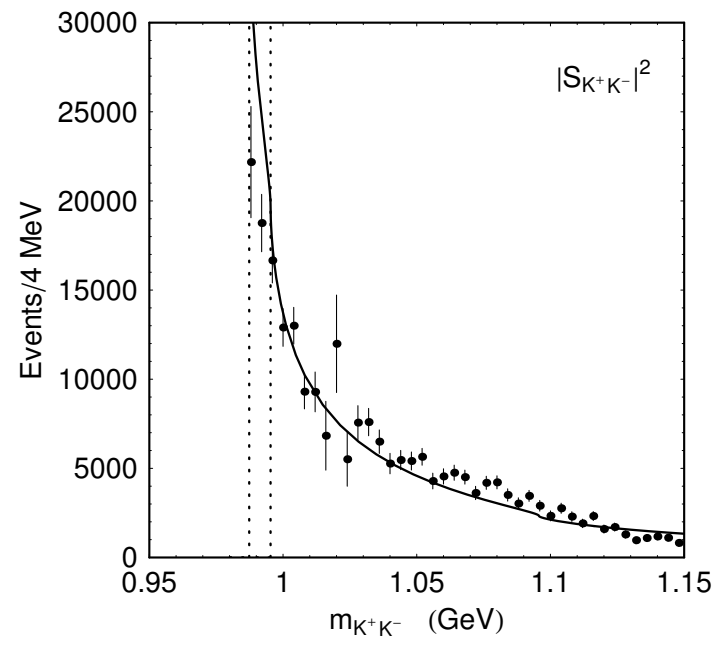

Figure 20: $S$-wave $K^{+} K^{-}$-mass spectrum in the decay $D_{s}^{+} \rightarrow$ $K^{+} K^{-} \pi^{+}$according to the BaBar data [156]; it corresponds to the absolute value squared of the transition amplitude and does not include the phase space factor of the $K^{+} K^{-}$system in $D_{s}^{+} \rightarrow K^{+} K^{-} \pi^{+}$. The vertical dotted lines show the location of the $K^{+} K^{-}$and $K^{0} \bar{K}^{0}$ thresholds. The solid curve corresponds to the contribution of the $f_{0}(980)$ resonance (see details in the text).

The shape of this spectrum and data on the shape of the $S$-wave $\pi^{+} \pi^{-}$mass spectrum in the decay $D_{s}^{+} \rightarrow$ $\pi^{+} \pi^{-} \pi^{+}$[157] can be successfully approximated by the contribution of the $f_{0}(980)$ resonance (see curves in Figs 20 and 21). Indeed, up to overall normalization constants, the curves reproduce the absolute value squared of the scalar resonance $f_{0}(980)$ propagator: $\left|S_{K^{+} K^{-}}\right|^{2} \sim$ $1 /\left|D_{f_{0}}\left(m_{K^{+} K^{-}}^{2}\right)\right|^{2}$, where $m_{K^{+} K^{-}}$is the $K^{+} K^{-}$invariant mass in the region above the $K^{+} K^{-}$threshold, $\left|S_{\pi^{+} \pi^{-}}\right|^{2} \sim 1 /\left|D_{f_{0}}\left(m_{\pi^{+} \pi^{-}}^{2}\right)\right|^{2}$, and $m_{\pi^{+} \pi^{-}}$is the $\pi^{+} \pi^{-}$ invariant mass. The $f_{0}(980)$ propagator itself was taken from [95] without any modifications.

According to the PDG [9], the decay probability is

$$
\begin{aligned}
B R\left(D_{s}^{+}\right. & \left.\rightarrow f_{0}(980) \pi^{+} \rightarrow K^{+} K^{-} \pi^{+}\right) \\
& =(1.15 \pm 0.32) \% .
\end{aligned}
$$

The value itself and its accuracy, which are based on the data of the BaBar [156] and CLEO [158] experiments, need further exploration. It was actually assumed in the initial analysis of BaBar 156 and CLEO 158 that the possible presence of the $a_{0}^{0}(980)$ resonance in the $K^{+} K^{-}$ system produced in the $D_{s}^{+} \rightarrow K^{+} K^{-} \pi^{+}$decay can be disregarded. Therefore, the value in (105) effectively corresponds to the sum of the $f_{0}(980)$ and $a_{0}^{0}(980)$ contributions to $D_{s}^{+}$-meson decay (the discussion of the data in [156 158] is continued in footnote 8 and at the end of this section).

The reliability of result (105) can be assessed by exploring the three-particle decays of the $D_{s}^{+}$meson, which are akin to $D_{s}^{+} \rightarrow K^{+} K^{-} \pi^{+}$. For example,

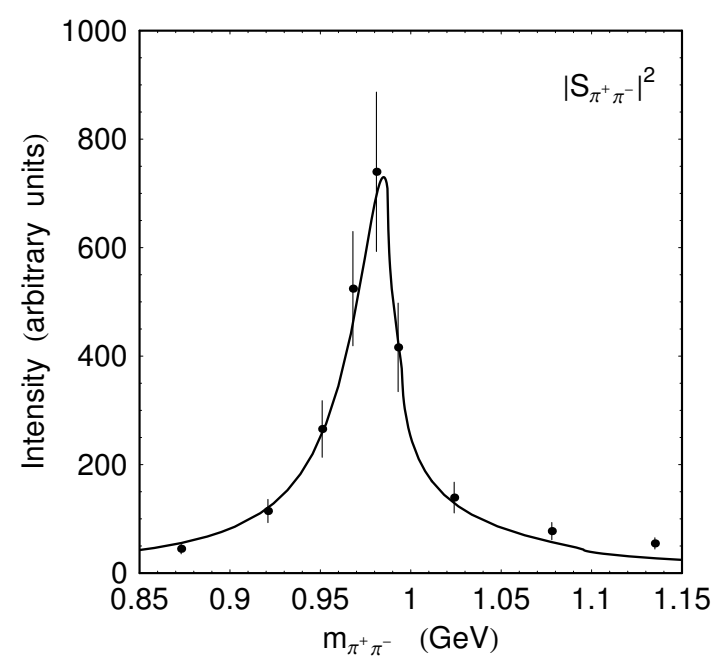

Figure 21: $S$-wave $\pi^{+} \pi^{-}$mass spectrum in the decay $D_{s}^{+} \rightarrow$ $\pi^{+} \pi^{-} \pi^{+}$according to the BaBar data [157]. The shape of the curve corresponds to the contribution of the $f_{0}(980)$ resonance (see details in the text).

we proposed in 140 to explore manifestations of the $a_{0}^{0}(980)-f_{0}(980)$ mixing in the $\eta \pi^{0}$ mass spectrum in the decay ${ }^{7} D_{s}^{+} \rightarrow \eta \pi^{0} \pi^{+}$to obtain additional information about the $f_{0}(980)$ and $a_{0}^{0}(980)$ resonance production mechanisms.

Guided by the value $B R\left(D_{s}^{+} \rightarrow f_{0}(980) \pi^{+} \rightarrow\right.$ $\left.K^{+} K^{-} \pi^{+}\right)$in (105) and the parameters of $f_{0}(980)$ and $a_{0}^{0}(980)$ resonances discussed above, we obtained the following estimate for the contribution to the decay $D_{s}^{+} \rightarrow$ $\eta \pi^{0} \pi^{+}$due to the $a_{0}^{0}(980)-f_{0}(980)$ mixing:

$$
\begin{gathered}
B R\left(D _ { s } ^ { + } \rightarrow \left[f_{0}(980) \rightarrow\left(K^{+} K^{-}+K^{0} \bar{K}^{0}\right)\right.\right. \\
\left.\left.\rightarrow a_{0}^{0}(980)\right] \pi^{+} \rightarrow \eta \pi^{0} \pi^{+}\right)=4.1 \times 10^{-4} .
\end{gathered}
$$

The corresponding amplitude of the transition $D_{s}^{+} \rightarrow$ $\left[f_{0}(980) \rightarrow\left(K^{+} K^{-}+K^{0} \bar{K}^{0}\right) \rightarrow a_{0}^{0}(980)\right] \pi^{+} \rightarrow \eta \pi^{0} \pi^{+}$ is presented in Eqn (110).

Data on the decay $D_{s}^{+} \rightarrow \eta \pi^{0} \pi^{+}[9,159,160]$ indicate that it is almost completely dominated by the $\eta \rho^{+}$ intermediate state:

$$
\begin{gathered}
B R\left(D_{s}^{+} \rightarrow \eta \rho^{+} \rightarrow \eta \pi^{0} \pi^{+}\right)=(8.9 \pm 0.8) \% \\
B R\left(D_{s}^{+} \rightarrow \eta \pi^{0} \pi^{+}\right)=(9.2 \pm 1.2) \%
\end{gathered}
$$

We let $A_{\eta \rho^{+}}$and $A_{f_{0} a_{0}^{0}}$ denote the amplitudes of the respective transitions $D_{s}^{+} \rightarrow \eta \rho^{+} \rightarrow \eta \pi^{0} \pi^{+}$and $D_{s}^{+} \rightarrow\left[f_{0}(980) \rightarrow\left(K^{+} K^{-}+\right.\right.$ $\left.\left.K^{0} \bar{K}^{0}\right) \rightarrow a_{0}^{0}(980)\right] \pi^{+} \rightarrow \eta \pi^{0} \pi^{+}$, and, to describe their

\footnotetext{
${ }^{7}$ It would be reasonable to explore the contributions of various intermediate states to the decay $D_{s}^{+} \rightarrow K_{S}^{0} K_{S}^{0} \pi^{+}$in detail.
} 
dependence on mass variables, use the expressions

$$
\begin{gathered}
A_{\eta \rho^{+}} \equiv A_{\eta \rho^{+}}\left(m_{\eta \pi^{0}}^{2}, m_{\eta \pi^{+}}^{2}, m_{\pi^{0} \pi^{+}}^{2}\right) \equiv A_{\eta \rho^{+}}(s, t, u) \\
=C_{D_{s}^{+} \eta \rho^{+}} \frac{s-t}{D_{\rho^{+}}(u)} F_{\rho}(u) \sqrt{\frac{g_{\rho \pi \pi}^{2}}{16 \pi}} \\
A_{f_{0} a_{0}^{0}} \equiv A_{f_{0} a_{0}^{0}}\left(m_{\eta \pi^{0}}^{2}\right) \equiv A_{f_{0} a_{0}^{0}}(s) \\
=C_{D_{s}^{+} f_{0} \pi^{+}} \frac{\Pi_{a_{0}^{0} f_{0}}(s)}{D_{a_{0}^{0}}(s) D_{f_{0}}(s)-\Pi_{a_{0}^{0} f_{0}}^{2}(s)} \sqrt{\frac{g_{a_{0}^{0} \eta \pi^{0}}^{2}}{16 \pi}},
\end{gathered}
$$

where $s=m_{\eta \pi^{0}}^{2}, t=m_{\eta \pi^{+}}^{2}$, and $u=m_{\pi^{0} \pi^{+}}^{2}$ are the invariant masses squared of the specified meson pairs in the decay $D_{s}^{+} \rightarrow \eta \pi^{0} \pi^{+}\left(\Sigma=s+t+u=m_{D_{s}}^{2}+2 m_{\pi}^{2}+\right.$ $\left.m_{\eta}^{2}\right) ; D_{\rho^{+}}(u)$ and $F_{\rho}(u)$ are the $\rho^{+}$-meson propagator and barrier factor in the decay $\rho^{+} \rightarrow \pi^{+} \pi^{0}$ [140]; $C_{D_{s}^{+} \eta \rho^{+}}$ and $C_{D_{s}^{+} f_{0} \pi^{+}}$are the invariant amplitudes of the decays $D_{s}^{+} \rightarrow \eta \rho^{+}$and $D_{s}^{+} \rightarrow f_{0}(980) \pi^{+}$. The effective vertices $D_{s}^{+} \rightarrow \eta \rho^{+}$and $\rho^{+} \rightarrow \pi^{0} \pi^{+}$were taken in the form

$$
\begin{gathered}
V_{D_{s}^{+} \eta \rho^{+}}=C_{D_{s}^{+} \eta \rho^{+}}\left(\epsilon_{\rho^{+}}^{*}, p_{D_{s}^{+}}+p_{\eta}\right) \\
V_{\rho^{+} \pi^{0} \pi^{+}}=g_{\rho \pi \pi}\left(\epsilon_{\rho^{+}}, p_{\pi^{+}}-p_{\pi^{0}}\right)
\end{gathered}
$$

where $\epsilon_{\rho^{+}}$is the $\rho^{+}$-meson polarization 4 -vector, and $p_{D_{s}^{+}}, p_{\eta}, p_{\pi^{0}}$, and $p_{\pi^{+}}$are the 4-momenta of the mesons $D_{s}^{+}, \eta, \pi^{0}$, and $\pi^{+}$in the decay $D_{s}^{+} \rightarrow \eta \pi^{0} \pi^{+}$. Thus, the kinematic factor $s-t$ in (109) is $\left(p_{D_{s}^{+}}+p_{\eta}, p_{\pi^{0}}-p_{\pi^{+}}\right)$. The amplitude $A_{f_{0} \pi}$ that is responsible for the decay $D_{s}^{+} \rightarrow f_{0}(980) \pi^{+} \rightarrow K^{+} K^{-} \pi^{+}[$see (105)] is given by

$$
\begin{gathered}
A_{f_{0} \pi} \equiv A_{f_{0} \pi}\left(m_{K^{+} K^{-}}^{2}\right) \equiv A_{f_{0} \pi}(s) \\
=C_{D_{s}^{+} f_{0} \pi^{+}} \frac{1}{D_{f_{0}}(s)} \sqrt{\frac{g_{f_{0} K^{+} K^{-}}^{2}}{16 \pi}} .
\end{gathered}
$$

Each invariant amplitude $C_{D_{s}^{+} \eta \rho^{+}}$and $C_{D_{s}^{+} f_{0} \pi^{+}}$is described by a pair of real numbers, the absolute value and phase, which do not depend on mass variables: $C_{D_{s}^{+} \eta \rho^{+}}=a_{1} e^{i \varphi_{1}}$ and $C_{D_{s}^{+} f_{0} \pi^{+}}=a_{2} e^{i \varphi_{2}}$. A similar approximation for the amplitudes of heavy-quarkonium decays containing light resonances in intermediate states is widely used for fitting distributions of Dalitz plot events (see, e.g., [156 158]). It is specifically this approximation that we use to obtain estimates.

As follows from (105) and (107),

$$
\frac{\left|C_{D_{s}^{+} f_{0} \pi^{+}}\right|}{\left|C_{D_{s}^{+} \eta \rho^{+}}\right|}=\frac{a_{2}}{a_{1}} \approx 4.5 \mathrm{GeV} .
$$

The $\eta \pi^{0}$ and $\pi^{0} \pi^{+}$mass spectra for the decay $D_{s}^{+} \rightarrow$ $\eta \pi^{0} \pi^{+}$, which correspond to a noncoherent sum of contributions from two mechanisms, $D_{s}^{+} \rightarrow \eta \rho^{+} \rightarrow \eta \pi^{0} \pi^{+}$and $D_{s}^{+} \rightarrow\left[f_{0}(980) \rightarrow\left(K^{+} K^{-}+K^{0} \bar{K}^{0}\right) \rightarrow a_{0}^{0}(980)\right] \pi^{+} \rightarrow$ $\eta \pi^{0} \pi^{+}$, are plotted in Figs $22 \mathrm{a}$ and $\mathrm{b}$ taking this relation into consideration. The emergence of the sharp peak with a width $\approx 2\left(m_{K^{0}}-m_{K^{+}}\right) \approx 8 \mathrm{MeV}$ at $m_{\eta \pi^{0}}$ in the region of the $K^{+} K^{-}$and $K^{0} \bar{K}^{0}$ thresholds in Fig. $22 \mathrm{a}$ is due to the $\eta \pi^{0}$-production mechanism owing to $a_{0}^{0}(980)-f_{0}(980)$ mixing. Figures $22 \mathrm{c}$ and d show, as an example, the distributions of approximately $10^{4}$ Monte Carlo events on $s-u$ and $s-t$ Dalitz diagrams for the decay $D_{s}^{+} \rightarrow \eta \pi^{0} \pi^{+}$, which also correspond to the hypothetical case of noncoherent addition of the two mechanisms. As follows from Eqn (109), the $s-u$ and $s$ - $t$ distributions for the mechanism $D_{s}^{+} \rightarrow \eta \rho^{+} \rightarrow \eta \pi^{0} \pi^{+}$vanish on the dotted lines $u=m_{D_{s}}^{2}+2 m_{\pi}^{2}+m_{\eta}^{2}-2 s$ and $t=s$ respectively plotted in Figs $22 \mathrm{c}$ and $\mathrm{d}$. Half of the $D_{s}^{+} \rightarrow \eta \rho^{+} \rightarrow \eta \pi^{0} \pi^{+}$events are located to the left and half to the right of these lines. The events that are due to the $a_{0}^{0}(980)-f_{0}(980)$ mixing concentrate in $s-u$ and $s-t$ Dalitz diagrams at $s=m_{\eta \pi^{0}}^{2} \approx 4 m_{K}^{2}$; they constitute about $1 \%$ of the half of the $D_{s}^{+} \rightarrow \eta \rho^{+} \rightarrow \eta \pi^{0} \pi^{+}$ events [see (106) and (107)]. This value is too high for the noncoherent isospin-violating contribution. As was noted above, it would be natural to expect that contributions of that type to the reaction amplitude are of the order of $\left(m_{d}-m_{u}\right) / \bar{m}$, where $m_{d}$ and $m_{u}$ are the masses of constituent quarks, $\bar{m}=\left(m_{d}+m_{u}\right) / 2$ [or of the order of the electromagnetic constant $\left.\alpha=e^{2} / 4 \pi\right]$, and hence of the order of $10^{-4}$ to the absolute value of the amplitude squared.

The mass spectrum of the $\eta \pi^{0}$ system in the decay $D_{s}^{+} \rightarrow \eta \pi^{0} \pi^{+}$in the case of coherent addition of the amplitude $A_{\eta \rho^{+}}$of the $D_{s}^{+} \rightarrow \eta \rho^{+} \rightarrow \eta \pi^{0} \pi^{+}$transition and the amplitude $A_{f_{0} a_{0}^{0}}$ due to the $a_{0}^{0}(980)-f_{0}(980)$ mixing has the form

$$
\frac{d N_{\eta \pi^{0}}}{d m_{\eta \pi^{0}}}=\int\left|A_{\eta \rho^{+}}+A_{f_{0} a_{0}^{0}}\right|^{2} 2 m_{\eta \pi^{0}} d m_{\pi^{0} \pi^{+}}^{2},
$$

where integration is performed over the physical domain of the variable $m_{\pi^{0} \pi^{+}}^{2}=u$. The relative phase of the amplitudes $C_{D_{s}^{+} f_{0} \pi^{+}}$and $C_{D_{s}^{+} \eta \rho^{+}}$becomes of importance here: $\varphi_{21}=\varphi_{2}-\varphi_{1}$ [or the parameter $\left.\xi=e^{i \varphi_{21}}\right]$. This phase is not known, and to illustrate possible outcomes of interference between the amplitudes $A_{\eta \rho^{+}}$and $A_{f_{0} a_{0}^{0}}$, we set it equal to $0^{\circ}, \pm 90^{\circ}$, and $180^{\circ}$ (correspondingly, $\xi=1, \pm i$ and -1$)$. The short and long dashed lines in Fig. 23a show the version of the $\eta \pi^{0}$ mass spectrum in the region of the $K^{+} K^{-}$and $K^{0} \bar{K}^{0}$ thresholds that correspond to the respective values $\xi=1$ and $\xi=-1$; the dotted line shows the contribution of the amplitude $A_{\eta \rho^{+}}$alone, and the solid curve represents the case of noncoherent addition of the two mechanisms that was discussed above. The dotted and solid lines in Fig. 23b show the same as in Fig. 23a, and the short and long dashed curves display the versions that correspond to the respective values $\xi=i$ and $\xi=-i$. It is clear that the interference of the amplitude $A_{f_{0} a_{0}^{0}}$ with other contributions virtually always manifests itself owing to rapid change of the phase of the $a_{0}^{0}(980)-f_{0}(980)$ transition in the region between the $K^{+} K^{-}$and $K^{0} \bar{K}^{0}$ thresholds (Fig. 2b).

As was noted in [140], the decay $D_{s}^{+} \rightarrow \eta \pi^{0} \pi^{+}$can oc- 

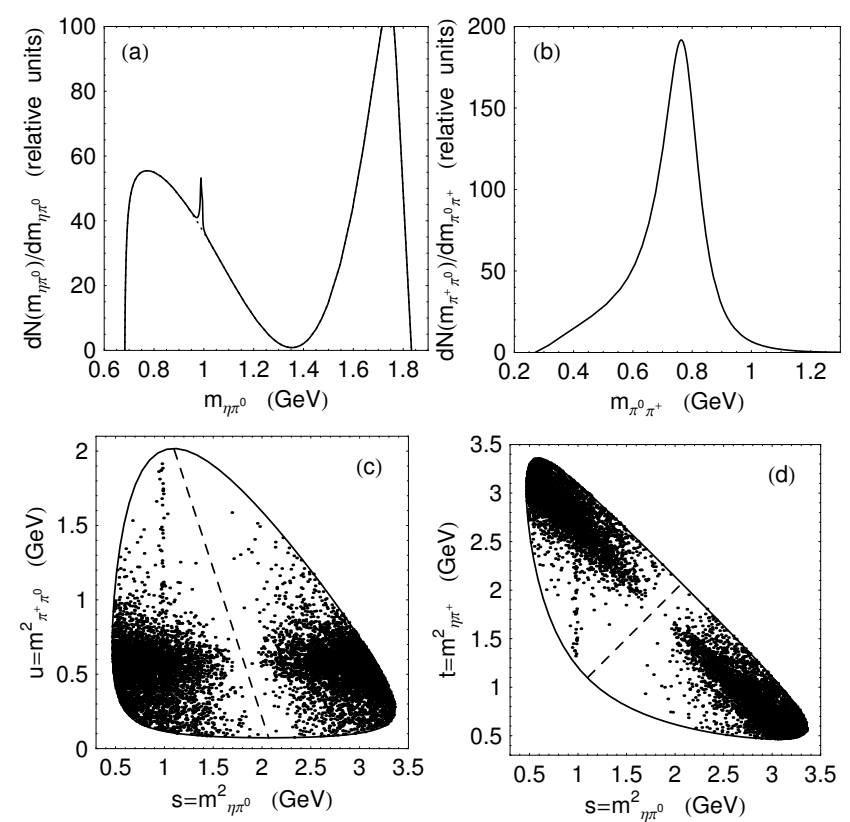

Figure 22: Illustration of a manifestation of $a_{0}^{0}(980)-$ $f_{0}(980)$ mixing in the decay $D_{s}^{+} \rightarrow \eta \pi^{0} \pi^{+}$on the background of its main mechanism $D_{s}^{+} \rightarrow \eta \rho^{+} \rightarrow$ $\eta \pi^{0} \pi^{+}$in the case of noncoherent addition of the contributions from $D_{s}^{+} \rightarrow \eta \rho^{+} \rightarrow \eta \pi^{0} \pi^{+}$and $D_{s}^{+} \rightarrow$ $\left[f_{0}(980) \rightarrow\left(K^{+} K^{-}+K^{0} \bar{K}^{0}\right) \rightarrow a_{0}^{0}(980)\right] \pi^{+} \rightarrow \eta \pi^{0} \pi^{+}$. (a, b) Mass spectra of the $\eta \pi^{0}$ and $\pi^{0} \pi^{+}$systems in the decay $D_{s}^{+} \rightarrow \eta \pi^{0} \pi^{+}$. (c, d) Examples of Monte Carlo distributions of the $D_{s}^{+} \rightarrow \eta \pi^{0} \pi^{+}$decay events in $s-u$ and $s-t$ Dalitz diagrams, respectively.

cur not only via the $\eta \rho^{+}$intermediate state or owing to the $a_{0}^{0}(980)-f_{0}(980)$ mixing but also via the intermediate state $\left(a_{0}(980) \pi\right)^{+}: D_{s}^{+} \rightarrow\left[a_{0}^{+}(980) \pi^{0}+a_{0}^{0}(980) \pi^{+}\right] \rightarrow$ $\eta \pi^{0} \pi^{+}$. We can expect, however, that the probability of that transition is not large. Using the data in (107) and (108) as a very rough (upper) estimate, we find $B R\left(D_{s}^{+} \rightarrow\left(a_{0}(980) \pi\right)^{+} \rightarrow \eta \pi^{0} \pi^{+}\right) \approx 1 \%{ }^{8}$ The case of three different mutually interfering mechanisms of the decay $D_{s}^{+} \rightarrow \eta \pi^{0} \pi^{+}$seems to be more realistic in principle. The corresponding combined amplitude of the decay is

$$
A_{D_{s}^{+} \rightarrow \eta \pi^{0} \pi^{+}}=A_{\eta \rho^{+}}+A_{f_{0} a_{0}^{0}}+A_{a_{0} \pi}
$$

where $A_{a_{0} \pi}$ is the amplitude of the transition $D_{s}^{+} \rightarrow$ $\left(a_{0}(980) \pi\right)^{+} \rightarrow \eta \pi^{0} \pi^{+}$, which [similarly to the amplitude $A_{\eta \rho^{+}}$; see (109)] is (in the isotopic-invariance approximation) antisymmetric with respect to permutations of $s$

\footnotetext{
8 The upper bound of $B R\left(D_{s}^{+} \rightarrow a_{0}^{0}(980) \pi^{+} \rightarrow K^{+} K^{-} \pi^{+}\right)$ is, in this case, $\approx 0.1 \%$. It is noteworthy that this estimate agrees with the initial assumption about the dominance of the $D_{s}^{+} \rightarrow f_{0}(980) \pi^{+} \rightarrow K^{+} K^{-} \pi^{+}$transition involving the $f_{0}(980)$ resonance [156 158] [see Eqn (105)].
}
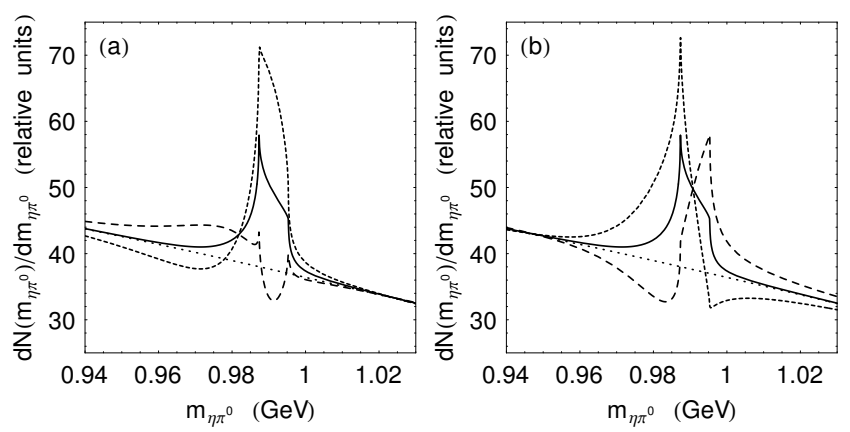

Figure 23: $\eta \pi^{0}$ mass spectra in the region of the $K^{+} K^{-}$and $K^{0} \bar{K}^{0}$ thresholds for four versions of interference between the amplitudes $D_{s}^{+} \rightarrow \eta \rho^{+} \rightarrow \eta \pi^{0} \pi^{+}$ and $D_{s}^{+} \rightarrow\left[f_{0}(980) \rightarrow\left(K^{+} K^{-}+K^{0} \bar{K}^{0}\right) \rightarrow a_{0}^{0}(980)\right] \pi^{+} \rightarrow$ $\eta \pi^{0} \pi^{+}$. These versions are compared with the spectrum in the noncoherent case. All the curves are described in the text.

and $t$ [140]. Given this feature, the amplitude $A_{a_{0} \pi}$ can be naturally approximated using the formula

$$
\begin{aligned}
& A_{a_{0} \pi} \equiv A_{a_{0} \pi}\left(m_{\eta \pi^{0}}^{2}, m_{\eta \pi^{+}}^{2}\right) \equiv A_{a_{0} \pi}(s, t) \\
= & C_{D_{s}^{+} a_{0}^{0} \pi^{+}}\left[\frac{1}{D_{a_{0}^{0}}(s)}-\frac{1}{D_{a_{0}^{+}}(t)}\right] \sqrt{\frac{g_{a_{0} \eta \pi^{0}}^{2}}{16 \pi}},
\end{aligned}
$$

where the amplitude $C_{D_{s}^{+} a_{0}^{0} \pi^{+}}=a_{3} e^{i \varphi_{3}}$ is assumed to be a complex constant independent of $s$ and $t$. We note that on the $s-t$ Dalitz diagram, any coherent sum of the amplitudes $A_{\eta \rho^{+}}$and $A_{a_{0} \pi}$ yields a distribution of $\eta \pi^{0} \pi^{+}$ events that is symmetric with respect to the $t=s$ line (and vanishes on the line itself.) Therefore, any asymmetry in the distribution of $\eta \pi^{0} \pi^{+}$events in the $s-t$ Dalitz diagram (with respect to the $t=s$ line) is only due to the $A_{f_{0} a_{0}^{0}}=A_{f_{0} a_{0}^{0}}(s)$ amplitude, which depends on $s$ alone, manifests itself in the region of the $K \bar{K}$ thresholds, and is generated by the isospin-violating $a_{0}^{0}(980)-f_{0}(980)$ mixing. An example of such an asymmetric $s-t$ distribution not involving the $A_{a_{0} \pi}$ amplitude is shown in Fig. $22 \mathrm{~d}$. A variety of examples of $\eta \pi^{0}$ mass spectra and asymmetric distributions of the $D_{s}^{+} \rightarrow \eta \pi^{0} \pi^{+}$decay events in the $s-t$ Dalitz diagrams, which illustrate possible scenarios of interference among amplitudes $A_{\eta \rho^{+}}, A_{f_{0} a_{0}^{0}}$, and $A_{a_{0} \pi}$ described by Eqns (109), (110), and (116), are reported in [140]. Interested readers are referred to that study for details.

Finding signatures of the mechanisms of the decay $D_{s}^{+} \rightarrow\left(a_{0}^{0}(980) \pi^{+}+a_{0}^{+}(980) \pi^{0}\right) \rightarrow \eta \pi^{0} \pi^{+}$is a challenging task for the physics of both weak hadronic decays of $D_{s}^{+}$mesons and light scalar $a_{0}(980)$ and $f_{0}(980)$ mesons. Progress in these areas in the nearest future will naturally be related to studies conducted by the $\mathrm{LHCb}$, BaBar, CLEO, BESIII, Belle, and Belle II facilities.

It is clear that the observation of $a_{0}^{0}(980)-f_{0}(980)$ mixing in the decay $D_{s}^{+} \rightarrow \eta \pi^{0} \pi^{+}$would provide independent information about the mechanism of production 
of $f_{0}(980)$ mesons (or the $K \bar{K}$ system with the isospin $I=0$ in an $S$-wave) and would therefore facilitate elucidating its role in the decay channel $D_{s}^{+} \rightarrow K^{+} K^{-} \pi^{+}$. This observation seems to be even more important in relation to another phenomenon that has not been explained yet. If $f_{0}(980)$ is produced in the $\pi^{+} \pi^{-}$and $K^{+} K^{-}$channels as an isolated resonance without any background, the CLEO result $B R\left(D_{s}^{+} \rightarrow \pi^{+} \pi^{-} \pi^{+}\right)=$ $(1.11 \pm 0.04 \pm 0.04) \%$ [160] (see also [9, 157]) disagrees with the assumption made by BaBar [156] and CLEO [158] that $f_{0}(980)$ dominates in the decay $D_{s}^{+} \rightarrow f_{0}(980) \pi^{+} \rightarrow$ $K^{+} K^{-} \pi^{+}$. We recall that the PDG's average value is $B R\left(D_{s}^{+} \rightarrow f_{0}(980) \pi^{+} \rightarrow K^{+} K^{-} \pi^{+}\right)=(1.15 \pm 0.32) \%$ [see (105)]. Our estimates show that agreement with that value and the shape of the line in Fig. 21 can be reproduced with $B R\left(D_{s}^{+} \rightarrow f_{0}(980) \pi^{+} \rightarrow \pi^{+} \pi^{-} \pi^{+}\right) \approx 3.4 \%$. The probability of the decay of an isolated $f_{0}(980)$ resonance into $\pi^{+} \pi^{-}$is higher than that into $K^{+} K^{-}$simply because of the $K^{+} K^{-}$system phase-space factor that suppresses the $f_{0}(980) \rightarrow K^{+} K^{-}$decay near the threshold. The situation undoubtedly requires further exploration to elucidate the $f_{0}(980)$ resonance production mechanisms in the decays $D_{s}^{+} \rightarrow f_{0}(980) \pi^{+} \rightarrow \pi^{+} \pi^{-} \pi^{+}$ and $D_{s}^{+} \rightarrow f_{0}(980) \pi^{+} \rightarrow K^{+} K^{-} \pi^{+}$.

\subsection{Decays $D^{0} \rightarrow K_{S}^{0} \pi^{+} \pi^{-}$and $D^{0} \rightarrow K_{S}^{0} \eta \pi^{0}$}

Studies of the $a_{0}^{0}(980)-f_{0}(980)$ mixing in three-particle decays of $D^{0}$ mesons, $D^{0} \rightarrow K_{S}^{0} \pi^{+} \pi^{-}, D^{0} \rightarrow K_{S}^{0} \eta \pi^{0}$, $D^{0} \rightarrow \bar{K}^{0} K^{-} K^{+}, D^{0} \rightarrow K^{-} K^{+} \pi^{0}$, and $D^{0} \rightarrow \pi^{+} \pi^{-} \pi^{0}$, are both promising and challenging. We here analyze possible manifestations of that mixing in the decays $D^{0} \rightarrow K_{S}^{0} \pi^{+} \pi^{-}$and $D^{0} \rightarrow K_{S}^{0} \eta \pi^{0}$ [141] and show that the $\pi^{+} \pi^{-}$mass spectrum in the decay $D^{0} \rightarrow K_{S}^{0} \pi^{+} \pi^{-}$is most strongly affected by the $a_{0}^{0}(980)-f_{0}(980)$ mixing. Owing to that mixing, the shape of the $f_{0}(980)$ peak in the decay $D^{0} \rightarrow K_{S}^{0} f_{0}(980) \rightarrow K_{S}^{0} \pi^{+} \pi^{-}$can experience deformations comparable to its magnitude. It is of importance that this effect significantly depends on the relative phase of the $D^{0} \rightarrow K_{S}^{0} f_{0}(980)$ and $D^{0} \rightarrow K_{S}^{0} a_{0}^{0}(980)$ decay amplitudes.

In the $D^{0} \rightarrow K_{S}^{0} \pi^{+} \pi^{-}$and $D^{0} \rightarrow K_{S}^{0} \eta \pi^{0}$ decays, the $\pi^{+} \pi^{-}$and $\eta \pi^{0}$ mass spectra that are due to the contribution of $f_{0}(980)$ and $a_{0}^{0}(980)$ resonances with the $a_{0}^{0}(980)-f_{0}(980)$ mixing taken into account are given by

$$
\begin{aligned}
& \frac{d N_{\pi^{+} \pi^{-}}}{d m}=2 m^{2} P_{K_{S}^{0}}(m) \Gamma_{f_{0} \rightarrow \pi^{+} \pi^{-}}(m) \\
& \times\left|\frac{C_{1}}{D_{f_{0}}(m)}+\frac{e^{i \varphi} C_{2} \Pi_{a_{0}^{0} f_{0}}(m)}{D_{a_{0}^{0}}(m) D_{f_{0}}(m)-\Pi_{a_{0}^{0} f_{0}}^{2}(m)}\right|^{2}, \\
& \frac{d N_{\eta \pi^{0}}}{d m}=2 m^{2} P_{K_{S}^{0}}(m) \Gamma_{a_{0}^{0} \eta \pi^{0}}(m) \\
& \times\left|\frac{e^{i \varphi} C_{2}}{D_{a_{0}^{0}}(m)}+\frac{C_{1} \Pi_{a_{0}^{0} f_{0}}(m)}{D_{a_{0}^{0}}(m) D_{f_{0}}(m)-\Pi_{a_{0}^{0} f_{0}}^{2}(m)}\right|^{2},
\end{aligned}
$$

where $m$ is the invariant mass of the $\pi^{+} \pi^{-}$or $\eta \pi^{0}$ system,

$P_{K_{S}^{0}}(m)=\frac{\left[m_{D^{0}}^{4}-2 m_{D^{0}}^{2}\left(m_{K^{0}}^{2}+m^{2}\right)+\left(m_{K^{0}}^{2}-m^{2}\right)^{2}\right]^{1 / 2}}{2 m_{D^{0}}}$,

and $\varphi$ is the relative phase between the $D^{0} \rightarrow K_{S}^{0} a_{0}^{0}(980)$ and $D^{0} \rightarrow K_{S}^{0} f_{0}(980)$ decay amplitudes. We have set the values of the constants $C_{1}=0.047 \mathrm{GeV}^{-1 / 2}$ and $C_{2}=0.095 \mathrm{GeV}^{-1 / 2}$ given the CLEO Data [161, 162], PDG information [9], and the relations

$$
\begin{aligned}
& B R\left(D^{0} \rightarrow K_{S}^{0} f_{0}(980) \rightarrow K_{S}^{0} \pi^{+} \pi^{-}\right) \\
& =\left(1.23_{-0.24}^{+0.40}\right) \times 10^{-3} \\
& =\int_{2 m_{\pi}^{+}}^{m_{D^{0}}-m_{K^{0}}} 2 P_{K_{S}^{0}}(m) \Gamma_{f_{0} \rightarrow \pi^{+} \pi^{-}}(m)\left|\frac{m C_{1}}{D_{f_{0}}(m)}\right|^{2} d m \\
& B R\left(D^{0} \rightarrow K_{S}^{0} a_{0}^{0}(980) \rightarrow K_{S}^{0} \eta \pi^{0}\right) \\
& =(6.6 \pm 2.0) \times 10^{-3} \\
& =\int_{m_{\eta}+m_{\pi^{0}}}^{m_{D^{0}}-m_{K^{0}}} 2 P_{K_{S}^{0}}(m) \Gamma_{a_{0}^{0} \eta \pi^{0}}(m)\left|\frac{m C_{2}}{D_{a_{0}^{0}}(m)}\right|^{2} d m,
\end{aligned}
$$

in which the same parameters as before are used for the $f_{0}(980)$ and $a_{0}^{0}(980)$ resonances.

We discuss the situation with decays that are of interest to us in more detail below. We only note here that the numerical values for the decay probabilities used in (119) and (120) are based on limited experimental statistics and an analysis of decay amplitudes in the isobar model. More accurate information about these values is definitely quite desirable. Unfortunately, in processing more recent experimental data on the $D^{0} \rightarrow K_{S}^{0} \pi^{+} \pi^{-}$ decay, the BaBar [163] and Belle [164] collaborations (see also [165]) have not specifically separated the $f_{0}(980)$ resonance contribution from the entire array of $S$-wave contributions. Nevertheless, the $f_{0}(980)$ peak is clearly seen in the $\pi^{+} \pi^{-}$mass spectrum in this decay [163, 164].

We define the fractions of isospin-violating contributions to the decays $D^{0} \rightarrow K_{S}^{0} \pi^{+} \pi^{-}$and $D^{0} \rightarrow K_{S}^{0} \eta \pi^{0}$ due to the $a_{0}^{0}(980)-f_{0}(980)$ mixing as

$$
\begin{aligned}
& \Delta B R\left(\pi^{+} \pi^{-}\right)=\frac{1}{1.23 \cdot 10^{-3}} \int_{2 m_{\pi}^{+}}^{m_{D^{0}}-m_{K^{0}}} \frac{d N_{\pi^{+} \pi^{-}}}{d m} d m-1, \\
& \Delta B R\left(\eta \pi^{0}\right)=\frac{1}{6.6 \cdot 10^{-3}} \int_{m_{\eta}+m_{\pi^{0}}}^{m_{D^{0}}-m_{K^{0}}} \frac{d N_{\eta \pi^{0}}}{d m} d m-1 . \quad(122)
\end{aligned}
$$

The quantities $\Delta B R\left(\pi^{+} \pi^{-}\right)$and $\Delta B R\left(\eta \pi^{0}\right)$ as functions of the relative phase $\varphi$ between the $D^{0} \rightarrow K_{S}^{0} a_{0}^{0}(980)$ and $D^{0} \rightarrow K_{S}^{0} f_{0}(980)$ decay amplitudes are shown in Fig. 24 by respective solid and dashed lines. The horizontal solid 


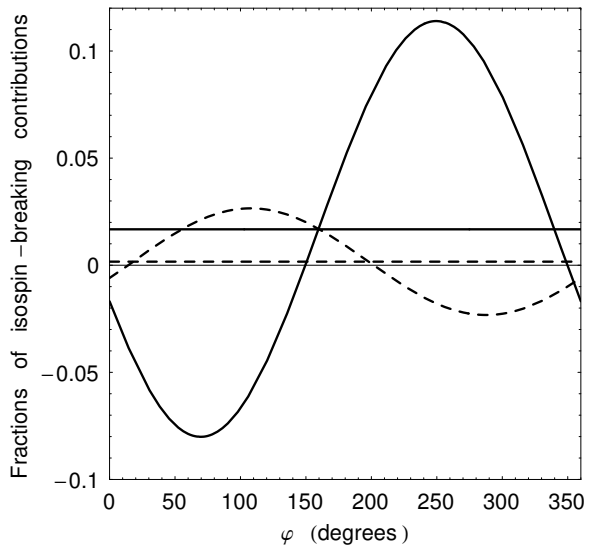

Figure 24: Dependences of the isospin-violating contributions $\Delta B R\left(\pi^{+} \pi^{-}\right)$(solid curve) and $\Delta B R\left(\eta \pi^{0}\right)$ (dashed curve) on the phase $\varphi$. The solid and dashed horizontal lines show the respective noncoherent contributions from the $a_{0}^{0}(980)-f_{0}(980)$ mixing in the $\pi^{+} \pi^{-}$and $\eta \pi^{0}$ channels.

and dashed lines in the same figure show the noncoherent contributions to $\Delta B R\left(\pi^{+} \pi^{-}\right)$and $\Delta B R\left(\eta \pi^{0}\right)$ from the $a_{0}^{0}(980)-f_{0}(980)$ mixing, i.e., the contributions due to the absolute values squared of the second summands in (117) and (118), which amount to $\approx 1.7 \%$ in the $\pi^{+} \pi^{-}$ channel and $\approx 0.17 \%$ in the $\eta \pi^{0}$ channel.

As follows from Fig. 24, the maximum constructive (destructive) interference of the contribution from $a_{0}^{0}(980)-f_{0}(980)$ mixing in the $\pi^{+} \pi^{-}$channel corresponds to the phase $\varphi \approx 245^{\circ}\left(70^{\circ}\right)$, and the maximum constructive (destructive) interference of the contribution from $a_{0}^{0}(980)-f_{0}(980)$ mixing in the $\eta \pi^{0}$ channel corresponds to the phase $\varphi \approx 110^{\circ}\left(290^{\circ}\right)$. Figure 25 shows the $d N_{\pi^{+} \pi^{-}} / d m$ and $d N_{\eta \pi^{0}} / d m$ mass spectra for these limit interference cases.

The branching ratio $B R\left(D^{0} \rightarrow K_{S}^{0} a_{0}^{0}(980) \rightarrow K_{S}^{0} \eta \pi^{0}\right)$ being approximately five times larger than $B R\left(D^{0} \rightarrow\right.$ $K_{S}^{0} f_{0}(980) \rightarrow K_{S}^{0} \pi^{+} \pi^{-}$) [see (119) and (120)] results in an enhancement of the $a_{0}^{0}(980)-f_{0}(980)$ mixing effect in the $\pi^{+} \pi^{-}$channel compared with this effect in the $\eta \pi^{0}$ channel. Owing to the interference, the integral effect of the $a_{0}^{0}(980)-f_{0}(980)$ mixing in the $\pi^{+} \pi^{-}$channel can be as high as $\approx 11 \%$ (see Fig. 24), a value that is too large for an isospin-violating effect. We can see from Fig. 25 that in the $\pi^{+} \pi^{-}$mass spectrum, the $a_{0}^{0}(980)-f_{0}(980)$ mixing can result in a significant deformation of the shape of the $f_{0}(980)$ resonance peak, for example, narrowing by a factor of approximately 1.5, and increasing its height by up to $60 \%$, or even in the emergence of two peaks. The effect significantly depends on the relative phase of the $D^{0} \rightarrow K_{S}^{0} f_{0}(980)$ and $D^{0} \rightarrow K_{S}^{0} a_{0}^{0}(980)$ decay amplitudes. To detect these fine phenomena, high resolution in the invariant mass $\mathrm{m}$ and large statistics of events are certainly needed.

We now discuss the experimental situation 9, 161[166]. The only experiment where the $D^{0} \rightarrow K_{S}^{0} \eta \pi^{0}$ decay
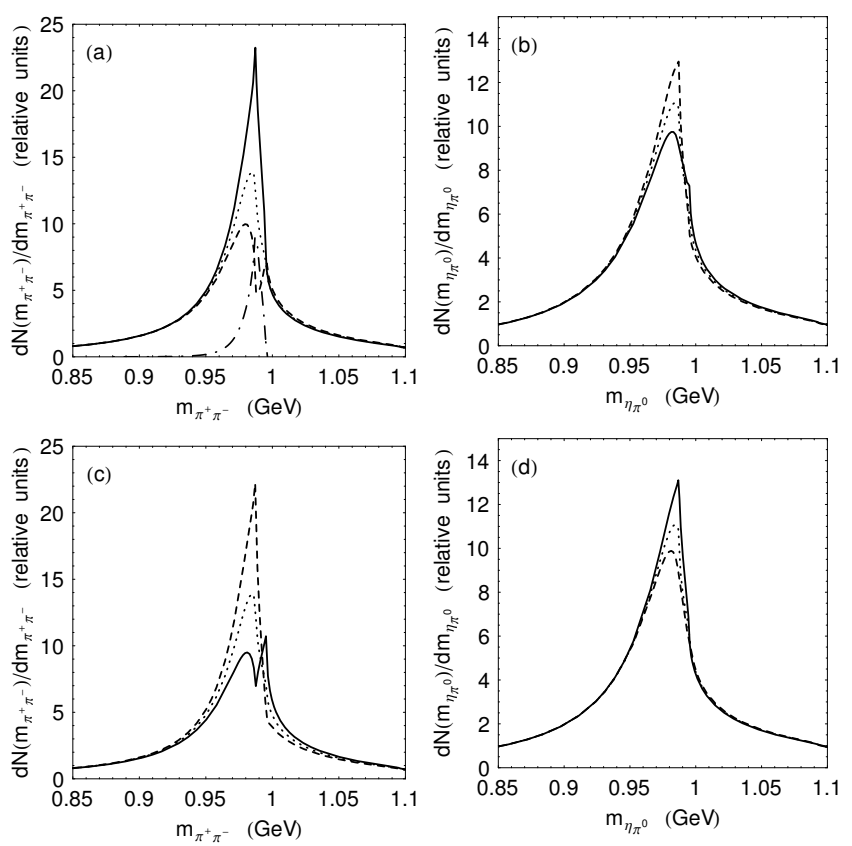

Figure 25: Mass spectra of $\pi^{+} \pi^{-}$(a) and $\eta \pi^{0}$ (b) at $\varphi=245^{\circ}$ (solid curves) and $\varphi=70^{\circ}$ (dashed curves); the dotted lines in Figs a and b correspond to spectra without the $a_{0}^{0}(980)-$ $f_{0}(980)$ mixing, i.e., contributions from the respective $f_{0}(980)$ and $a_{0}^{0}(980)$ resonances. (c, d) The same as in Fig. a and b but for $\varphi=110^{\circ}$ (solid curves) and $\varphi=290^{\circ}$ (dashed curves). The dashed-dotted curve that corresponds to the difference between the values of the solid and dashed curves is shown as an example in Fig. a.

has been observed was performed by the CLEO collaboration [162] with statistics from 155 events. An analysis of the distribution of these events in the Dalitz diagram showed in [162] that the $D^{0} \rightarrow K_{S}^{0} \eta \pi^{0}$ decay primarily occurs via the $K_{S}^{0} a_{0}^{0}(980)$ and $K^{* 0}(892) \eta$ intermediate states, of which the former dominates. It is noteworthy that $a_{0}^{0}(980)$ production was observed not only in the decay $D^{0} \rightarrow K_{S}^{0} a_{0}^{0}(980) \rightarrow K_{S}^{0} \eta \pi^{0}$ (for which $B R\left(D^{0} \rightarrow\right.$ $\left.K_{S}^{0} a_{0}^{0}(980) \rightarrow K_{S}^{0} \eta \pi^{0}\right)=(6.6 \pm 2.0) \times 10^{-3}[9,[162])$ but also in the channel $D^{0} \rightarrow K_{S}^{0} a_{0}^{0}(980) \rightarrow K_{S}^{0} K^{+} K^{-}$ [165, 166]. According to data in [166], $B R\left(D^{0} \rightarrow\right.$ $\left.K_{S}^{0} a_{0}^{0}(980) \rightarrow K_{S}^{0} K^{+} K^{-}\right)=(3.0 \pm 0.4) \times 10^{-3}$, while it follows from the supplementary material in 165] (see Ref. [18] there) that the central value is $B R\left(D^{0} \rightarrow\right.$ $\left.K_{S}^{0} a_{0}^{0}(980) \rightarrow K_{S}^{0} K^{+} K^{-}\right) \approx 2.3 \times 10^{-3}$. It is noteworthy that the quoted branching ratios of the decays $D^{0} \rightarrow K_{S}^{0} a_{0}^{0}(980) \rightarrow K_{S}^{0} \eta \pi^{0}[9]$ and $D^{0} \rightarrow K_{S}^{0} a_{0}^{0}(980) \rightarrow$ $K_{S}^{0} K^{+} K^{-}$agree with the $q^{2} \bar{q}^{2}$ model of the $a_{0}^{0}(980)$ resonance [3, 7, 18].

The number of candidates for the events of the $D^{0} \rightarrow$ $K_{S}^{0} \pi^{+} \pi^{-}$decay selected in the CLEO [161], BaBar [163], and Belle [164] experiments was 5,299, 487,000, and $1,231,731$, respectively. The Dalitz distributions of the $D^{0} \rightarrow K_{S}^{0} \pi^{+} \pi^{-}$events exhibit a rich structure. The list of possible intermediate states includes $K^{*-}(982) \pi^{+}$, 
$K^{*-}(1430) \pi^{+}, \quad K_{S}^{0} \rho^{0}, \quad K_{S}^{0} f_{0}(980), \quad K_{S}^{0} f_{2}(1270), \quad$ and $K_{S}^{0} f_{0}(1370)$. An estimate in [161] shows that the contribution of the $K_{S}^{0} f_{0}(980) \rightarrow K_{S}^{0} \pi^{+} \pi^{-}$channel is $\left(4.3_{-0.8}^{+1.4}\right) \%$. In total, production of $K_{S}^{0}$ together with the $S$-wave $\pi^{+} \pi^{-}$system, $K_{S}^{0}\left(\pi^{+} \pi^{-}\right)_{S}$, yields about $12 \%$ of the $D^{0} \rightarrow K_{S}^{0} \pi^{+} \pi^{-}$decay probability [163, 164]. The $\pi^{+} \pi^{-}$invariant-mass step in the mass region of $1 \mathrm{GeV}$ was about $5 \mathrm{MeV}$ in the BaBar [163] and Belle [164] experiments. It is noteworthy that the entire visible $f_{0}(980)$ peak contains 6 to 7 points, implying that its width is less than $25 \mathrm{MeV}$. The narrowness of the $f_{0}(980)$ peak can be related to the effect of $a_{0}^{0}(980)-f_{0}(980)$ mixing. However, interference with the background from other intermediate states certainly cannot be ruled out a priori. Future studies will hopefully resolve this issue. The most clear-cut information about events corresponding to the $D^{0} \rightarrow K_{S}^{0} f_{0}(980) \rightarrow K_{S}^{0} \pi^{+} \pi^{-}$channel and the possible influence of background events on that channel comes from the distribution of $D^{0} \rightarrow K_{S}^{0} \pi^{+} \pi^{-}$events in the $\left(m_{\pi^{+} \pi^{-}}^{2}, m_{K_{S}^{0} \pi^{ \pm}}^{2}\right)$ Dalitz diagram.

\section{Bottomonium decay $\Upsilon(10860) \rightarrow$ $\Upsilon(1 S) f_{0}(980) \rightarrow \Upsilon(1 S) \eta \pi^{0}$}

The Belle collaboration has recently performed a complete amplitude analysis of three-particle transitions $e^{+} e^{-} \rightarrow \Upsilon(n S) \pi^{+} \pi^{-}(n=1,2,3)$ for the energy $\sqrt{s}=$ $10.865 \mathrm{GeV}$ in the $e^{+} e^{-}$c.m.s. and determined the relative weight of different quasi-two-particle components of the three-particle amplitudes and the spin and parity of two observed $Z_{b}$ states [167]. ${ }^{9}$ The first data on the transition $e^{+} e^{-} \rightarrow \Upsilon(1 S) f_{0}(980)$ have also been reported. According to [167], the fraction of the decay $\Upsilon(10860) \rightarrow \Upsilon(1 S) f_{0}(980)$ is

$$
\begin{gathered}
\frac{B R\left(\Upsilon(10860) \rightarrow \Upsilon(1 S) f_{0}(980) \rightarrow \Upsilon(1 S) \pi^{+} \pi^{-}\right)}{B R\left(\Upsilon(10860) \rightarrow \Upsilon(1 S) \pi^{+} \pi^{-}\right)} \\
=\left(6.9 \pm 1.6_{-2.8}^{+0.8}\right) \%
\end{gathered}
$$

Apart from decaying into $\pi^{+} \pi^{-}$, the resonance $f_{0}(980)$ can also decay into $\eta \pi^{0}$ via the transition

$$
f_{0}(980) \rightarrow\left(K^{+} K^{-}+K^{0} \bar{K}^{0}\right) \rightarrow a_{0}^{0}(980) \rightarrow \eta \pi^{0},
$$

i.e., owing to the $a_{0}^{0}(980)-f_{0}(980)$ mixing. Guided by the central value of the strength of the $f_{0}(980) \rightarrow a_{0}^{0}(980)$ transition measured in the BES III experiment for the reaction $J / \psi \rightarrow \phi f_{0}(980) \rightarrow \phi a_{0}^{0}(980) \rightarrow \phi \eta \pi^{0}$ [see (34)], i.e., assuming that

$$
\frac{B R\left(f_{0}(980) \rightarrow K \bar{K} \rightarrow a_{0}^{0}(980) \rightarrow \eta \pi^{0}\right)}{B R\left(f_{0}(980) \rightarrow \pi^{+} \pi^{-}\right)} \approx 0.009
$$

\footnotetext{
9 We are grateful to A E Bondar', who directed our attention to the decay $\Upsilon(10860) \rightarrow \Upsilon(1 S) f_{0}(980) \rightarrow \Upsilon(1 S) \eta \pi^{0}$ and data from the Belle collaboration [167].
}

we arrive at the following estimate for the fraction of isospin- violating decays of the $\Upsilon(10860)$ meson [168]:

$$
\begin{gathered}
\frac{B R\left(\Upsilon(10860) \rightarrow \Upsilon(1 S) f_{0}(980) \rightarrow \Upsilon(1 S) \eta \pi^{0}\right)}{B R\left(\Upsilon(10860) \rightarrow \Upsilon(1 S) \pi^{+} \pi^{-}\right)} \\
\approx 6.2 \times 10^{-4} .
\end{gathered}
$$

We note that the $f_{0}(980)$ resonance in the $\pi^{+} \pi^{-}$mass spectrum in the decay $\Upsilon(10860) \rightarrow \Upsilon(1 S) \pi^{+} \pi^{-}$was observed in [167] not as a peak but as a deep dip due to destructive interference with a large and smooth background. The dominance of a narrow resonance peak in the $\eta \pi^{0}$ mass spectrum in the region of $K \bar{K}$ thresholds is supposed to be a characteristic feature of the decay $\Upsilon(10860) \rightarrow \Upsilon(1 S) f_{0}(980) \rightarrow \Upsilon(1 S) \eta \pi^{0}$ because there is no obvious background in the $\eta \pi^{0}$ channel of the decay $\Upsilon(10860) \rightarrow \Upsilon(1 S) \eta \pi^{0}$. The $\eta \pi^{0}$ spectrum is [168]

$$
\begin{aligned}
\frac{d N\left(\Upsilon(5 S) \rightarrow \Upsilon(1 S) \eta \pi^{0}\right)}{d m}=C p(m) \frac{2 m^{2} \Gamma_{a_{0}^{0} \rightarrow \eta \pi^{0}}(m)}{\pi} \\
\quad \times\left|\frac{\Pi_{a_{0}^{0} f_{0}}(m)}{D_{a_{0}^{0}}(m) D_{f_{0}}(m)-\Pi_{a_{0}^{0} f_{0}}^{2}(m)}\right|^{2}
\end{aligned}
$$

where $\Upsilon(5 S)$ is a shorthand notation for $\Upsilon(10860), m$ is the invariant mass of the $\eta \pi^{0}$ system, $p(m)$ is the $\eta \pi^{0}$ momentum in the $\Upsilon(5 S)$ rest frame, and $C$ is a normalization constant. The momentum $p(m)$ is weakly dependent on $m$ in the mass range $m \sim 1 \mathrm{GeV}$ in the decay $\Upsilon(5 S) \rightarrow \Upsilon(1 S) \eta \pi^{0}$, and therefore the spectrum virtually coincides with the $\eta \pi^{0}$ mass spectrum shown by the solid curve in Fig. 3.

The number of events selected as candidates for the decay $\Upsilon(10860) \rightarrow \Upsilon(1 S) \pi^{+} \pi^{-}$in the Belle experiment under discussion in [167] was 2,090土115. Thus, one or two events corresponding to the decay $\Upsilon(10860) \rightarrow \Upsilon(1 S) \eta \pi^{0}$ could be produced owing to the $a_{0}(980)-f_{0}(980)$ mixing in the course of that experiment. One may hope that the Belle II detector at the $e^{+} e^{-}$collider SuperKEK B to be launched shortly will allow measuring this rare decay with an accuracy of no worse than 10 percent. Given the instantaneous luminosity $8 \times 10^{35} \mathrm{~cm}^{-2} \mathrm{~s}^{-1}$ of the SuperKEK B collider (40 times larger than that of KEK), the Bell detector will be able to record approximately $100 \Upsilon(10860) \rightarrow \Upsilon(1 S) \eta \pi^{0}$ events (i.e., from 50 to 150 events) in a new experiment in a narrow region of the $\eta \pi^{0}$ invariant mass near the $K \bar{K}$ thresholds within a timeframe that was needed for the initial experiment of the Belle collaboration [167]. ${ }^{10}$ This implies that the anomalous violation of isotopic invariance will enable studying the production mechanisms and nature of light scalar mesons also in the bottomonium domain.

In conclusion, we make a comment that is primarily addressed to experimentalists. In obtaining result

\footnotetext{
${ }^{10}$ We estimate that about three months was needed to collect the
} statistics in the experiment [167]. 


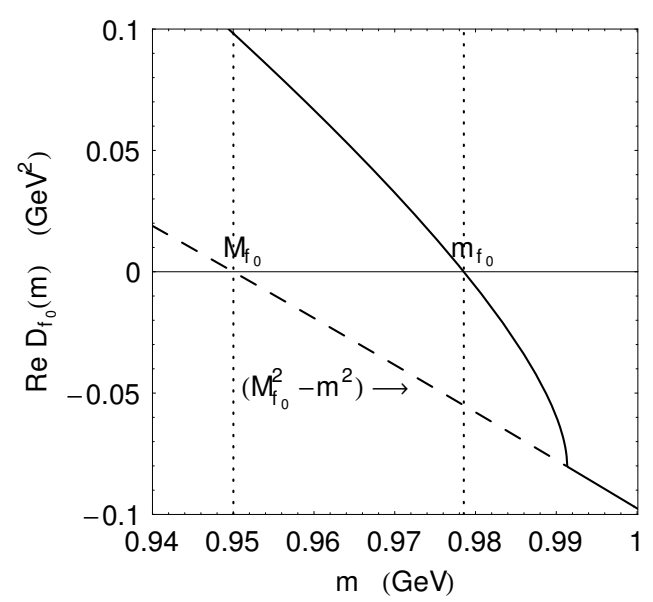

Figure 26: Illustration $m_{f_{0}}$ shift to values larger than $M_{f_{0}}$. The solid curve shows $\operatorname{Re} D_{f_{0}}(m)=$ $\operatorname{Re}\left(M_{f_{0}}^{2}-m^{2}+M_{f_{0}} g_{K \bar{K}}\left(m_{K}^{2}-m^{2} / 4\right)^{1 / 2}\right)$ as a function of $m$.

(123) for the relative fraction of the decay $\Upsilon(10860) \rightarrow$ $\Upsilon(1 S) f_{0}(980) \rightarrow \Upsilon(1 S) \pi^{+} \pi^{-}$, the Belle collaboration [167] used the Flatté propagator for the $f_{0}(980)$ resonance [10, 12],

$$
\frac{1}{D_{f_{0}}(m)}=\frac{1}{M_{f_{0}}^{2}-m^{2}-i M_{f_{0}}\left(g_{\pi \pi} q_{\pi}+g_{K \bar{K}} q_{K}\right)}
$$

where $q_{\pi}=\sqrt{m^{2} / 4-m_{\pi}^{2}}$ and

$$
q_{K}= \begin{cases}\sqrt{m^{2} / 4-m_{K}^{2}}, & \text { above the } K \bar{K} \text { threshold, } \\ i \sqrt{m_{K}^{2}-m^{2} / 4}, & \text { below the } K \bar{K} \text { threshold, }\end{cases}
$$

with the mass $M_{f_{0}}=950 \mathrm{MeV}$ and constants $g_{\pi \pi}=0.23$ and $g_{K \bar{K}}=0.75$ obtained in [169 from the analysis of data on the $B^{ \pm} \rightarrow K^{ \pm} \pi^{ \pm} \pi^{ \pm}$decay. The mass $M_{f_{0}}=$ $950 \mathrm{MeV}$, which is $30-40 \mathrm{MeV}$ smaller than the value in tables [9], may be perplexing. However, we should take into account that at $M_{f_{0}}<2 m_{K}$, the value of $M_{f_{0}}^{2}$ in the Flatté formula does not coincide with the location of a zero of the real part of the resonance propagator, in contrast to the case of the resonance mass squared if the generally accepted definition is used [see, e.g., the definition of $m_{f_{0}}$ in Eqn (5)]. The value of $m_{f_{0}}$ as the location of a zero of $\operatorname{Re} D_{f_{0}}(m)$ should be determined from the equation

$$
\operatorname{Re}\left(M_{f_{0}}^{2}-m^{2}+M_{f_{0}} g_{K \bar{K}} \sqrt{m_{K}^{2}-\frac{m^{2}}{4}}\right)=0 .
$$

We find $m_{f_{0}} \approx 979 \mathrm{MeV}$ from this equation. Figure 26 clearly shows that $m_{f_{0}}$ is shifted to values larger than $M_{f_{0}}$.

It has been noted many times that the Flatté propagators are not satisfactory for studying $f_{0}(980)$ and $a_{0}(980)$ resonances [41, 433, 97, 170]. If these propagators are employed for the $f_{0}(980)$ and $a_{0}(980)$ states with masses below the $K \bar{K}$ thresholds (see examples in 9, 156, 167, 169, 171, 172]), the resonance masses found by fitting must be renormalized. Our propagators [see (5)-(9)], which were first proposed in [2], are not plagued with this problem. Therefore, we once again recommend using them to determine the parameters of $f_{0}(980)$ and $a_{0}(980)$ mesons.

\section{Reactions violating isotopic invariance in the central region}

The exclusive reactions of hadron production in the central region ${ }^{11}$ of high-energy $p p$ collisions, $p p \rightarrow p\left(X^{0}\right) p$, were studied at the ISR (Intersecting Storage Rings) and $S p \bar{p} S$ (Super Proton-Antiproton Synchrotron) at CERN and the Tevatron at the Fermi National Accelerator Laboratory (Fermilab) using a fixed target, and are currently being explored at the Large Hadron Collider (LHC) at CERN (see, e.g., reviews [24, 173 176]). Mass spectra and production cross sections have been measured in these experiments for a number of hadronic systems $X^{0}: \pi \pi$ 176 [181], $K \bar{K}$ [179, 182 184], $\eta \pi^{+} \pi^{-}$[185, 186], $K \bar{K} \pi$ [187, 188], $4 \pi$ [189, 190], $\eta \pi^{0}$ [191], etc. Special attention was paid to studying resonance contributions.

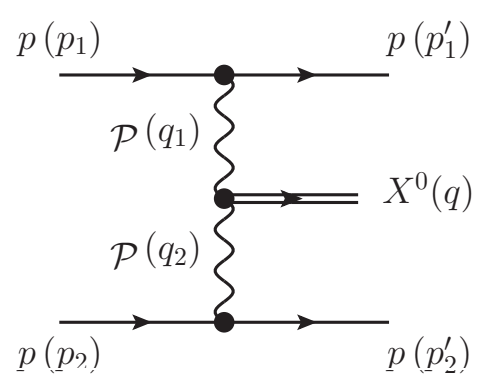

Figure 27: Production of the $X^{0}$ hadron system in the central region in the reaction $p p \rightarrow p\left(X^{0}\right) p$ due to the double pomeron exchange, $\mathcal{P} \mathcal{P}$. Indicated in parentheses are the 4 momenta of the initial and final protons, $\mathcal{P}$-exchanges, and the $X^{0}$-system; the main kinematic variables in this reaction are $s=\left(p_{1}+p_{2}\right)^{2}, s_{1}=\left(p_{1}^{\prime}+q\right)^{2}, s_{2}=\left(p_{2}^{\prime}+q\right)^{2}$, $M^{2}=q^{2}=\left(q_{1}+q_{2}\right)^{2}$, and $t_{1}=q_{1}^{2}, t_{2}=q_{2}^{2}$.

Resonances are produced in the central region at high energies primarily due to the double pomeron exchange (Fig. 27). Only resonances with positive $C$-parity and the isotopic spin $I=0$ can be produced in a two-pomeron collision. The cross sections of processes driven by that mechanism do not decrease as a power of energy 24, 173, 192 194]. Therefore, the observation of resonances in $X^{0}$ states with $I=1$ is a signal that they are produced or decay with isotopic invariance violation. Below, we

11 The value of the Feynman variable $x_{F} \approx 0$ can be considered a common feature of the particles produced in the central region. 
present examples where such situations can occur [195].

\subsection{Reactions $p p \rightarrow p\left(f_{1}(1285) / f_{1}(1420)\right) p \rightarrow$ $p\left(\pi^{+} \pi^{-} \pi^{0}\right) p$}

Clear-cut signals from $f_{1}(1285)$ and $f_{1}(1420)$ resonances (with $\left.I^{G}\left(J^{P C}\right)=0^{+}\left(1^{++}\right)\right)$produced in the central region of the reaction $p p \rightarrow p\left(f_{1}(1285) / f_{1}(1420)\right) p \rightarrow$ $p\left(X^{0}\right) p$ have been observed in all of their main decay modes: $\eta \pi \pi$ [185, 186], $K \bar{K} \pi$ [175, 187, 188], and $4 \pi$ [189]. Experiments were performed at the momenta $P_{l a b}^{p}=85,300,450$, and $800 \mathrm{GeV} / c$ of the protons incident on a fixed target, or, correspondingly, at the full energy $\sqrt{s}=12.7,23.8,29$, and $40 \mathrm{GeV}$ in the reaction center-of-mass system. Data on the production cross sections of these resonances are compatible with the $\mathcal{P} \mathcal{P}$-exchange mechanism [174, 175, 185 189]. An important complementary experimental observation is that the central-region production of states with $I^{G}\left(J^{P C}\right)=$ $0^{+}\left(0^{-+}\right)$and masses around 1.28 and $1.4 \mathrm{GeV}$ was fully suppressed [174, 175, 185 189]. In practical terms, this circumstance may be helpful in determining the properties of the $f_{1}(1285)$ and $f_{1}(1420)$ resonances more accurately than in other experiments where states with both $J^{P C}=1^{++}$and $J^{P C}=0^{-+}$are concurrently observed.

Thus, in studying the production of $f_{1}(1285)$ and $f_{1}(1420)$ in the central region in $p p$ collisions, it is possible to determine the probabilities of decays of the two resonances into all of their main modes in a single experiment. We also note that due to the isotopic neutrality of $\mathcal{P} \mathcal{P}$ exchange, the reaction $p p \rightarrow p\left(f_{1}(1285)\right) p \rightarrow$ $p\left(\pi^{+} \pi^{-} \pi^{0}\right) p$ provides a unique possibility of studying the isospin-violating decay $f_{1}(1285) \rightarrow f_{0}(980) \pi^{0} \rightarrow$ $\pi^{+} \pi^{-} \pi^{0}$ in a setup that is virtually free of any background [195]. Owing to a substantially different arrangement of the experiment, such a study would be a very efficient test of the first results from VES [122] [see (32) and (33)] and BESIII [134] [see (52)], which indicate a very strong violation of isospin invariance in that decay. A search for isospin-violating events $p p \rightarrow p\left(\pi^{+} \pi^{-} \pi^{0}\right) p$ in the vicinity of the $f_{1}(1420)$ resonance is also of interest.

Regarding options to measure the reaction $p p \rightarrow$ $p\left(f_{1}(1285)\right) p \rightarrow p\left(\pi^{+} \pi^{-} \pi^{0}\right) p$, we note the following. In principle, one could use the data recorded by the Omega spectrometer at CERN and the CDF detector (Collider Detector at Fermilab) to extract information about the events $p p \rightarrow p\left(\pi^{+} \pi^{-} \pi^{0}\right) p$ in the central region. However, enthusiasts are needed for this task, because the facilities themselves were decommissioned long ago. The reaction $p p \rightarrow p\left(f_{1}(1285)\right) p \rightarrow p\left(\pi^{+} \pi^{-} \pi^{0}\right) p$ could currently be measured at the LHC using the CMS (Compact Muon Solenoid) detector. The CMS collaboration has recently reported data on $\pi^{+} \pi^{-}$production in the central region in $p p$ collisions at $\sqrt{s}=7 \mathrm{TeV}$ [176]. At an energy this immense, the energies $\sqrt{s_{1}}$ and $\sqrt{s_{2}}$ of the subprocesses $p\left(p_{1}\right) \mathcal{P}\left(q_{2}\right) \rightarrow p\left(p_{1}^{\prime}\right) X^{0}(q)$ and $p\left(p_{2}\right) \mathcal{P}\left(q_{1}\right) \rightarrow p\left(p_{2}^{\prime}\right) X^{0}(q)$ (see Fig. 27) are also very large (and correspond to the energy range where exchange by secondary Regge trajec-

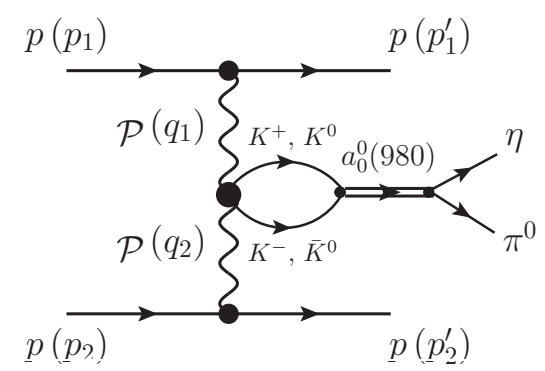

Figure 28: $K \bar{K}$-loop mechanism of $a_{0}^{0}(980)$ production in the central region in $\mathcal{P} \mathcal{P}$ collisions.

tories, $\mathcal{R}$, can de facto be disregarded compared to the $\mathcal{P}$ exchange). Setting $s_{1} \approx s_{2}, M \approx 1 \mathrm{GeV}$, and $\sqrt{s}=7$ $\mathrm{TeV}$ and using the relation $s_{1} s_{2} \approx M^{2} s$ (which holds for processes in the central region [192 194]), we obtain $\sqrt{s_{1}} \approx \sqrt{s_{2}} \approx 84 \mathrm{GeV}$. Thus, the dominance of the $\mathcal{P} \mathcal{P}$ exchange in LHC experiments is a very good approximation. We note for comparison that in the fixed-target experiments performed at CERN and the Tevatron, the corresponding values of $\sqrt{s_{1}} \approx \sqrt{s_{2}}$ were $\approx 3.6,4.9,5.4$, and $6.3 \mathrm{GeV}$. Therefore, in interpreting the experimental results, it was necessary in some cases to take possible contributions from $\mathcal{R} \mathcal{P}$ and $\mathcal{R} \mathcal{R}$ exchanges into account, in addition to the $\mathcal{P} \mathcal{P}$ exchange.

Production of the $\left.f_{1}(1285)\right)$ resonance and its subsequent decay into $\pi^{+} \pi^{-} \pi^{0}$ could also be studied in the central region of $p p, p A, \pi^{-} p$, and $\pi^{-} A$ collisions in the accelerator of the Institute of High Energy Physics in Protvino.

\subsection{Reaction $p p \rightarrow p\left(a_{0}^{0}(980)\right) p \rightarrow p\left(\eta \pi^{0}\right) p$}

The $a_{0}^{0}(980)$ resonance production at LHC energies in the central region in the reaction $p p \rightarrow p\left(a_{0}^{0}(980)\right) p \rightarrow$ $p\left(\eta \pi^{0}\right) p$ is supposed to primarily occur owing to the mechanism shown in Fig. 28 [195]. Due to an incomplete cancellation of the $K^{+} K^{-}$and $K^{0} \bar{K}^{0}$ intermediate states produced in $\mathcal{P} \mathcal{P}$ collisions, the isospin-violating amplitude of $a_{0}^{0}(980)$ production does not decrease as energy grows. The mass spectrum of the final $\eta \pi^{0}$ system should be a narrow resonance peak concentrated in the region of the $K \bar{K}$ thresholds, similarly to that depicted with a solid curve in Fig. 3. Judging by available data, the amplitude of the $\mathcal{P} \mathcal{P} \rightarrow K \bar{K}$ transition, which drives the process shown in Fig. 28, is dominated by the contribution from $f_{0}(980)$ resonance production 174, 175, 177-184], $\mathcal{P} \mathcal{P} \rightarrow f_{0}(980) \rightarrow K \bar{K}$ (Fig. 29).

Indeed, the mass spectra of $K^{+} K^{-}$and $K^{0} \bar{K}^{0}$ pairs exhibit a powerful enhancement in the vicinity of their thresholds [182 184]. The resonance $f_{0}(980)$ also clearly manifests itself in the $\pi^{+} \pi^{-}$and $\pi^{0} \pi^{0}$ mass spectra, albeit in the form of a narrow dip that occurs as a result of destructive interference with a large and smooth coherent background [174, 176 181. Thus, $a_{0}^{0}(980)$ is quite probably produced due to the $a_{0}^{0}(980)-f_{0}(980)$ mixing, $\mathcal{P P} \rightarrow f_{0}(980) \rightarrow\left(K^{+} K^{-}+K^{0} \bar{K}^{0}\right) \rightarrow a_{0}^{0}(980) \rightarrow \eta \pi^{0}$. 


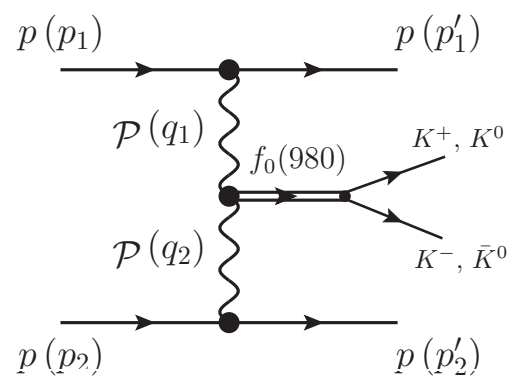

Figure 29: Production of the $f_{0}(980)$ resonance in $K \bar{K}$ decay channels in the central region in $\mathcal{P} \mathcal{P}$ collisions.

The corresponding cross section as a function of the invariant mass $M \equiv m$ of the $\eta \pi^{0}$ system is given by

$$
\begin{gathered}
\sigma\left(\mathcal{P P} \rightarrow f_{0}(980) \rightarrow\left(K^{+} K^{-}+K^{0} \bar{K}^{0}\right)\right. \\
\left.\rightarrow a_{0}^{0}(980) \rightarrow \eta \pi^{0} ; m\right)=\left|C_{\mathcal{P} \mathcal{P} \rightarrow f_{0}}\right|^{2} m \Gamma_{a_{0}^{0} \rightarrow \eta \pi^{0}}(m) \\
\times\left|\frac{\Pi_{a_{0}^{0} f_{0}}(m)}{D_{a_{0}^{0}}(m) D_{f_{0}}(m)-\Pi_{a_{0}^{0} f_{0}}^{2}(m)}\right|^{2}
\end{gathered}
$$

where $C_{\mathcal{P} \mathcal{P} \rightarrow f_{0}}$ is the $\mathcal{P} \mathcal{P} \rightarrow f_{0}(980)$ transition amplitude.

The $a_{0}^{0}(980)$ production cross section in $\mathcal{P P}$ collisions followed by the decay into $\eta \pi^{0}$ can be estimated without going into details of the transition $\mathcal{P} \mathcal{P} \rightarrow K \bar{K}$ (see Fig. $29)$. The latter can, in principle, be due to not only the contribution of the $f_{0}(980)$ resonance (see Fig. 29) but also a nonresonance mechanism of $K \bar{K}$ production.

For this, we use the relation

$$
\begin{gathered}
\sigma\left(\mathcal{P P} \rightarrow a_{0}^{0}(980) \rightarrow \eta \pi^{0} ; m\right) \\
\approx\left|\widetilde{A}\left(2 m_{K^{+}}\right)\right|^{2}\left|\rho_{K^{+} K^{-}}(m)-\rho_{K^{0} \bar{K}^{0}}(m)\right|^{2} \\
\times \frac{g_{a_{0}^{0} K^{+} K^{-}}^{2}}{16 \pi} \frac{m \Gamma_{a_{0}^{0} \rightarrow \eta \pi^{0}}(m)}{\left|D_{a_{0}^{0}}(m)\right|^{2}}
\end{gathered}
$$

in which $\left|\widetilde{A}\left(2 m_{K^{+}}\right)\right|^{2}$ should be determined using data on the $K^{+} K^{-}$production cross section in the near-threshold region:

$$
\sigma\left(\mathcal{P P} \rightarrow K^{+} K^{-} ; m\right)=\rho_{K^{+} K^{-}}(m)|\widetilde{A}(m)|^{2} .
$$

An order-of-magnitude estimate in the region of $m$ between the $K^{+} K^{-}$and $K^{0} \bar{K}^{0}$ thresholds yields

$$
\sigma\left(\mathcal{P P} \rightarrow a_{0}^{0}(980) \rightarrow \eta \pi^{0} ; m\right) \approx 0.05\left|\widetilde{A}\left(2 m_{K^{+}}\right)\right|^{2} .
$$

A comparison of this estimate with data on $\sigma(\mathcal{P P} \rightarrow$ $\left.a_{0}^{0}(980) \rightarrow \eta \pi^{0} ; m\right)$ allows verifying their consistency with data on $\sigma\left(\mathcal{P P} \rightarrow K^{+} K^{-} ; m\right)$ and the hypothesized violation of isotopic invariance due to the mass difference of $K^{+}$and $K^{0}$ mesons. It should be kept in mind that a similar way to check the consistency of the results of measurements for the decays $f_{1}(1285) \rightarrow \pi^{+} \pi^{-} \pi^{0}$ and $f_{1}(1285) \rightarrow K \bar{K} \pi$ was discussed in Section 5.2. Formulas relating $\sigma\left(\mathcal{P P} \rightarrow X^{0} ; m\right)$ to the experimentally measured cross section of the reaction $p p \rightarrow p\left(X^{0}\right) p$ can be found, e.g., in 192 194].

Data about the central production of $a_{0}^{0}(980)$ in the reaction $p p \rightarrow p\left(\eta \pi^{0}\right) p$ are only available from measurements made by the Omega spectrometer at CERN at $\sqrt{s}=29 \mathrm{GeV}$ [191, 196] (see the discussion on the interpretation of those data in 104, 107, 109, 123]). We note the following. Clear-cut peaks at $\sqrt{s}=29 \mathrm{GeV}$ have been observed in the $\eta \pi^{0}$ mass spectrum that correspond to $a_{0}^{0}(980)$ and $a_{2}^{0}(1320)$ resonances with widths typical of those mesons, $\Gamma\left(a_{0}(980)\right)=72 \pm 16 \mathrm{MeV}$ and $\Gamma\left(a_{2}(1320)\right)=115 \pm 20 \mathrm{MeV}$ (see PDG data [9]). This picture at the energies $\sqrt{s_{1}} \approx \sqrt{s_{2}} \approx\left(m_{a_{0}^{0}}^{2} s\right)^{1 / 4} \approx$ $\left(29^{2} \mathrm{GeV}^{4}\right)^{1 / 4} \approx 5.4 \mathrm{GeV}$ indicates a significant role of secondary Regge trajectories, for which the $\eta \pi^{0}$ production in the central region is not forbidden by $G$-parity. For example, the $a_{0}^{0}(980)$ resonance can be produced in merging the $\eta \pi^{0}, a_{2}^{0} f_{2}$, and $a_{2}^{0} \mathcal{P}$ Regge exchanges. Contributions from the secondary Regge trajectories significantly taper off at LHC energies, and it is natural to expect that the $a_{0}^{0}(980)$ resonance will only be produced owing to the mechanism described above (see Fig. 28), which does not 'fade' and violates isotopic invariance. The change in the mechanism of central production of $a_{0}^{0}(980)$ will be signalled by a narrowing of the $a_{0}^{0}(980)$ peak in the $\eta \pi^{0}$ channel with increasing energy.

\section{Conclusion}

The phenomenon of $a_{0}^{0}(980)-f_{0}(980)$ mixing [2] gave a boost to the experimental search for its effects in reactions (24)-(28) as performed by the VES [120, 122] and BES III 96, 125, 134] collaborations. These activities clearly showed that studies of the $a_{0}^{0}(980)-f_{0}(980)$ mixing in various reactions and a more general phenomenon, the $K \bar{K}$-loop mechanism of isotopic invariance violation, can be very helpful in identifying both the production mechanism of light scalar mesons and the nature of those mesons [1, [5].

We emphasize once again that the mass spectrum of light scalar mesons $\sigma(600), \kappa(800), a_{0}(980)$, and $f_{0}(980)$ indicates their four-quark, $q^{2} \bar{q}^{2}$, structure. The rates and mechanisms of the production of $a_{0}(980)$ and $f_{0}(980)$ resonances in radiative decays of the $\phi(1020)$ meson, four-quark transitions $\phi(1020) \rightarrow K^{+} K^{-} \rightarrow$ $\gamma\left[a_{0}(980) / f_{0}(980)\right]$, indicate their $q^{2} \bar{q}^{2}$ nature. The rates and mechanisms of two-photon production of light scalars, four-quark transitions $\gamma \gamma \rightarrow \pi^{+} \pi^{-} \rightarrow$ $\sigma(600), \gamma \gamma \rightarrow \pi^{0} \eta \rightarrow a_{0}(980)$, and $\gamma \gamma \rightarrow K^{+} K^{-} \rightarrow$ $f_{0}(980) / a_{0}(980)$, are also evidence in favor of their $q^{2} \bar{q}^{2}$ nature.

We also note that these states cannot be loosely bound molecules [1]. The decay $\phi(1020) \rightarrow K^{+} K^{-} \rightarrow$ $\gamma a_{0}^{0}(980) / f_{0}(980)$ was shown in [41, 65, 66] to involve virtual momenta of $K(\bar{K})$ mesons over $2 \mathrm{GeV} / c$, while in the case of loosely bound molecules with a binding energy of about $20 \mathrm{MeV}$ the corresponding momenta 
would be approximately $100 \mathrm{MeV} / c$. It is noteworthy that the production of scalar mesons in $\pi N$ collisions at large transferred momenta also indicates a compact structure of these particles [197]. Promising prospects are presented in [1] for studying the nature of $a_{0}(980)$ and $f_{0}(980)$ states in $\gamma \gamma$ and $\gamma \gamma^{*}$ collisions (where $\gamma^{*}$ is the virtual gamma-quantum), in $J / \psi$ decays, and in $\pi N$ collisions, and when comparing the production of light scalar and pseudoscalar mesons in semileptonic decays of $D_{s}$ and $D$ mesons in $\mathrm{c}-\tau$ and super-c- $-\tau$ factories and semileptonic decays of $B$ mesons in super-b factories.

The high statistical accuracy of modern-day experiments raises hopes that data on $a_{0}^{0}(980)-f_{0}(980)$ mixing will be elucidated (as confirmed by the recent study of the BESIII collaboration [198]), and new precise data on the decays $f_{1}(1285) \rightarrow f_{0}(980) \pi^{0} \rightarrow \pi^{+} \pi^{-} \pi^{0}$ and $\eta(1405) \rightarrow$ $f_{0}(980) \pi^{0} \rightarrow \pi^{+} \pi^{-} \pi^{0}$ will be obtained. The isotopic in- variance violation discussed in this review will hopefully be discovered in polarization experiments, weak hadronic decays of charmed $D_{s}^{+}$and $D^{0}$ mesons, the bottomonium decay $\Upsilon(10860) \rightarrow \Upsilon(1 S) f_{0}(980) \rightarrow \Upsilon(1 S) \eta \pi^{0}$, and the production of hadrons in the central region. It is quite probable that other interesting cases of strong isospin violation in the production of $a_{0}^{0}(980)$ and $f_{0}(980)$ resonances related to the difference in masses between $K^{+}$ and $K^{0}$ mesons will be observed, in particular, in $B$ and $B_{s}$ decays.

This study was partially supported by the grant no. 16-02-00065 from the Russian Foundation for Basis Research and the grant no. 0314-2019-0021 from Program no. II.15.1 of fundamental scientific research of the Siberian Branch of the Russian Academy of Sciences.
[1] Achasov N N Phys. Part. Nucl. 48681 (2017)

[2] Achasov N N, Devyanin S A, Shestakov G N Phys. Lett. $B$ 88, 367 (1979)

[3] Achasov N N, Devyanin S A, Shestakov G N Sov. J. Nucl. Phys. 33, 715 (1981); Yad. Fiz. 33, 1337 (1981)

[4] Achasov N N, Devyanin S A, Shestakov G N Sov. Phys. Usp. 27, 161 (1984); Usp. Fiz. Nauk 142, 361 (1984)

[5] Achasov N N, Shestakov G N Nuclear and Particle Physics Proceedings 287-288, 89 (2017)

[6] Achasov N N, Shestakov G N Sov. Phys. Usp. 34, 471 (1991); Usp. Fiz. Nauk 161, (6) 53 (1991)

[7] Achasov N N Phys. Usp. 41, 1149 (1998); Usp. Fiz. Nauk 168, 1257 (1998)

[8] Achasov N N, Shestakov G N Phys. Usp. 54, 799 (2011); Usp. Fiz. Nauk 181, 827 (2011)

[9] Tanabashi M et al. (Particle Data Group) Phys. Rev. D 98, 030001 (2018)

[10] Flatté S M et al. Phys. Lett. B 38, 232 (1972)

[11] Gay J B et al. Phys. Lett. B 63, 220 (1976)

[12] Flatté S M Phys. Lett. B 63, 224 (1976); Phys. Lett. B 63, 228 (1976)

[13] Jaffe R L Phys. Rev. D 15, 267, 281 (1977); Phys. Rev. D 15, 281 (1977)

[14] Martin A D, Ozmutlu E N, Squires E J Nucl. Phys. B 121, 514 (1977)

[15] Achasov N N, Devyanin S A, Shestakov G N Sov. J. Nucl. Phys. 32, 566 (1980); Yad. Fiz. 32, 1098 (1980)

[16] Achasov N N, Devyanin S A, Shestakov G N Phys. Lett. $B$ 96, 168 (1980)

[17] Achasov N N, Devyanin S A, Shestakov G N Z. Phys. C 22, 53 (1984)

[18] Achasov N N, Ivanchenko V N Nucl. Phys. B 315, 465 (1989)

[19] Close F E, Törnqvist N A J. Phys. G 28, R249 (2002)

[20] Amsler C, Törnqvist N A Phys. Rep. 389, 61 (2004)

[21] Maiani L et al. Phys. Rev. Lett. 93, 212002 (2004)

[22] Fariborz A H, Jora R, Schechter J, Phys. Rev. D 72, 034001 (2005)

[23] Jaffe R L Phys. Rep. 409, 1 (2005)

[24] Klempt E, Zaitsev A Phys. Rep. 454, 1 (2007)

[25] 't Hooft G et al. Phys. Lett. B 662, 424 (2008)
[26] Weinberg S Phys. Rev. Lett. 110, 261601 (2013)

[27] Dai L-Y, Pennington M R Phys. Rev. D 90, 036004 (2014)

[28] Peláez J R Phys. Rep. 658, 1 (2016)

[29] Wolkanowski T, Giacosa F, Rischke D H, Phys. Rev. D 93, 014002 (2016)

[30] Kim K S, Kim H Eur. Phys. J. C 77, 435 (2017)

[31] Gell-Mann M, Lévy M Nuovo Cim. 16, 705 (1960)

[32] Gell-Mann M Physics 1, 63 (1964)

[33] Lévy M Nuovo Cim. A 52, 23 (1967)

[34] Achasov N N, Shestakov G N Phys. Rev. D 49, 5779 (1994)

[35] Barnett R M et al. (Particle Data Group) Phys. Rev. D 54, 1 (1996)

[36] Eidelman S et al. (Particle Data Group) Phys. Lett. B 592, 1 (2004)

[37] Achasov N N, Shestakov G N Phys. Rev. Lett. 99, 072001 (2007)

[38] Bramon A, Grau A, Pancheri G Phys. Lett. B 289, 97 (1992)

[39] Close F E, Isgur N, Kumano S Nucl. Phys. B 389, 513 (1993)

[40] Lucio J L, Napsuciale M Phys. Lett. B 331, 418 (1994)

[41] Achasov N N, Gubin V V, Shevchenko V I Phys. Rev. D 56, 203 (1997)

[42] Achasov N N, Gubin V V, Solodov E P Phys. Rev. D 55, 2672 (1997)

[43] Achasov N N, Gubin V V Phys. Rev. D 56, 4084 (1997)

[44] Achasov N N, Gubin V V Phys. Rev. D 57, 1987 (1998)

[45] Achasov M N et al. (SND Collab.) Phys. Lett. B 438, 441 (1998)

[46] Achasov M N et al. (SND Collab.) Phys. Lett. B 440, 442 (1998)

[47] Achasov M N et al. (SND Collab.) Phys. Lett. B 479, $53(2000)$

[48] Achasov M N et al. (SND Collab.) Phys. Lett. B 485, 349 (2000)

[49] Akhmetshin R R et al. (CMD-2 Collab.) Phys. Lett. B 462, 371 (1999)

[50] Akhmetshin R R et al. (CMD-2 Collab.) Phys. Lett. B 462, 380 (1999) 
[51] Aloisio A et al. (KLOE Collab.) Phys. Lett. B 536, 209 (2002)

[52] Aloisio A et al. (KLOE Collab.) Phys. Lett. B 537, 21 (2002)

[53] Ambrosino F et al. (KLOE Collab.) Phys. Lett. B 634, 148 (2006)

[54] Ambrosino F et al. (KLOE Collab.) Eur. Phys. J. C 49, 473 (2007)

[55] Bossi F et al. (KLOE Collab.) Riv. Nuovo Cim. 031, 531 (2008)

[56] Ambrosino F et al. (KLOE Collab.) Phys. Lett. B 679, $10(2009)$

[57] Ambrosino F et al. (KLOE Collab.) Phys. Lett. B 681, 5 (2009)

[58] Achasov N N, Gubin V V Phys. Rev. D 63, 094007 (2001)

[59] Achasov N N, Gubin V V Phys. Rev. D 64, 094016 (2001)

[60] Achasov N N Phys. Atom. Nucl. 65, 546 (2002); Yad. Fiz. 65, 573 (2002)

[61] Achasov N N Nucl. Phys. A 728, 425 (2003)

[62] Achasov N N Phys. Atom. Nucl. 67, 1529 (2004); Yad. Fiz. 67, 1552 (2004)

[63] Achasov N N, Kiselev A V Phys. Rev. D 68, 014006 (2003)

[64] Achasov N N, Kiselev A V Phys. Rev. D 73, 054029 (2006)

[65] Achasov N N, Kiselev A V Phys. Rev. D 76, 077501 (2007)

[66] Achasov N N, Kiselev A V Phys. Rev. D 78, 058502 (2008)

[67] Achasov N N, Kiselev A V Phys. Rev. D 85, 094016 (2012)

[68] Achasov N N, Devyanin S A, Shestakov G N Phys. Lett. $B$ 108, 134 (1982)

[69] Achasov N N, Devyanin S A, Shestakov G N Z. Phys. C 16, 55 (1982)

[70] Antreasyan D et al. (Crystal Ball Collab.) Phys. Rev. D 33, 1847 (1986)

[71] Marsiske $\mathrm{H}$ et al. (Crystal Ball Collab.) Phys. Rev. D 41, 3324 (1990)

[72] Boyer J et al. (MARK II Collab.) Phys. Rev. D 42, 1350 (1990)

[73] Achasov N N, Shestakov G N Z. Phys. C 41, 309 (1988)

[74] Mori T et al. (Belle Collab.) Phys. Rev. D 75, 051101(R) (2007)

[75] Mori T et al. (Belle Collab.) J. Phys. Soc. Jpn. 76, 074102 (2007)

[76] Uehara S et al. (Belle Collab.) Phys. Rev. D 78, 052004 (2008)

[77] Uehara S et al. (Belle Collab.) Phys. Rev. D 80, 032001 (2009)

[78] Pennington M R et al. Eur. Phys. J. C 56, 1 (2008)

[79] Achasov N N, Shestakov G N Phys. Rev. D 72, 013006 (2005)

[80] Achasov N N, Shestakov G N Phys. Rev. D 77, 074020 (2008)

[81] Achasov N N, Kiselev A V Phys.Rev. D 86, 114010 (2012)

[82] Achasov N N, Kiselev A V Int. J. Mod. Phys. Conf. Ser. 35, 1460447 (2014)

[83] Asner D M et al. (BESIII Collab.) Int. J. Mod. Phys. A 24, S1 (2009)

[84] Alvarenga Nogueira J H et al. "Summary of the 2015
LHCb Workshop on Multi-Body Decays of $D$ and $B$ Mesons", arXiv:1605.03889

[85] dos Reis A C "LHCb-three-body decays of charged $D$ mesons", in Alvarenga Nogueira $\mathrm{J} \mathrm{H}$ et al., arXiv:1605.03889 Sec. IV, p. 13; "Amplitude analysis of charm decays at LHCb", in Proc. of the 8th Intern. Workshop on Charm Physics, 5-9 September, 2016, Bologna, Italy; PoS CHARM2016, 034 (2017)

[86] Loiseau B, in Proc. of the VIII International Workshop on Charm Physics, 5-9 September, 2016 Bologna, Italy; arXiv:1611.05286

[87] Achasov N N, Devyanin S A, Shestakov G N Sov. Phys. JETP Lett. 40, 1173 (1984); Pis'ma Zh. Eksp. Teor. 40, 365 (1984)

[88] Achasov N N, Devyanin S A, Shestakov G N Z. Phys. C 27, 99 (1985)

[89] Kolanoski H Two-photon Physics at $e^{+} e^{-}$Storage Rings (Tracts in Modern Physics, Vol. 105) (Berlin: SpringerVerlag, 1984)

[90] Albrecht H et al. (ARGUS Collab.) Phys. Lett. B 217, 205 (1989)

[91] Behrend H J et al. (CELLO Collab.) Phys. Lett. B 218, 493 (1989)

[92] Achasov N N, Shestakov G N Int. J. Mod. Phys. A 7, 4313 (1992)

[93] Achasov N N, Shestakov G N Phys. Rev. D 60, 114021 (1999)

[94] Ioffe B L Phys. Usp. 44, 1211 (2001); Usp. Fiz. Nauk 171, 1273 (2001)

[95] Achasov N N, Kozhevnikov A A, Shestakov G N Phys. Rev. D 93, 114027 (2016)

[96] Ablikim M et al. (BESIII Collab.) Phys. Rev. D 83, 032003 (2011)

[97] Achasov N N, Kiselev A V Phys. Rev. D 70, 111901(R) (2004)

[98] Achasov N N, Shestakov G N Phys. Rev. D 56, 212 (1997)

[99] Irving A C Nucl. Phys. B 105, 491 (1976)

[100] Dzierba A R, in Proc. of the Second Workshop on Physics and Detectors for DAFNE'95 (Eds R Baldini et al.) (Frascati Physics Ser., Vol. 4) (Frascati: INFN, 1996), p. 99

[101] Teige S et al. (E852 Collab.) Phys. Rev. D 59, 012001 (1998)

[102] Sadovsky S A, AIP Conf. Proc. 432, 774 (1998)

[103] Alde D et al. Phys. At. Nucl. 62, 421 (1999); Yad. Fiz. 62, 462 (1999)

[104] Achasov N N, Shestakov G N Phys. Rev. Lett. 92, 182001 (2004)

[105] Achasov N N, Shestakov G N Phys. Rev. D 70, 074015 (2004)

[106] Kerbikov B, Tabakin F Phys. Rev. C 62, 064601 (2000)

[107] Close F E, Kirk A Phys. Lett. B 489, 24 (2000)

[108] Grishina V Yu et al. Phys. Lett. B 521, 217 (2001)

[109] Achasov N N, Kiselev A V Phys. Lett. B 534, 83 (2002)

[110] Black D, Harada M, Schechter J Phys. Rev. Lett. 88, 181603 (2002)

[111] Kudryavtsev A E et al. Phys. Rev. C 66, 015207 (2002); Phys. At. Nucl. 66, 1946 (2003); Yad. Fiz. 66, 1994 (2003)

[112] Büscher M et al. arXiv:hep-ph/0301126

[113] Kondratyuk L A et al. Yad. Fiz. 66, 155 (2003) [Phys. At. Nucl. 66, 152 (2003)]

[114] Hanhart C AIP Conf. Proc. 688, 61 (2003); Phys. Rep. 
397, 155 (2004)

[115] Büscher M Acta Phys. Pol. B 35, 1055 (2004)

[116] Wang Z-G, Yang W-M, Wan S-L Eur. Phys. J. C 37, $223(2004)$

[117] Adam H-H et al. nucl-ex/0411038

[118] Hanhart C, Kubis B, Peláez J R Phys. Rev. D 76, 074028 (2007)

[119] Nikolaenko V, in Proc. of the 12th Intern. Conf. on Hadron Spectroscoty (Frascati Physics Series Vol. 46, Eds L Benussi et al.) (Frascati: INFN, 2007), p. 617

[120] Dorofeev V et al. (VES Collab.) Eur. Phys. J. A 38, 149 (2008)

[121] Nikolaenko V et al. (VES Collab.) Int. J. Mod. Phys. A 24, 295 (2009)

[122] Dorofeev V et al. (VES Collab.) Eur. Phys. J. A 47, 68 (2011)

[123] Wu J-J, Zhao Q, Zou B S Phys. Rev. D 75, 114012 (2007)

[124] Wu J-J, Zou B S Phys. Rev. D 78, 074017 (2008)

[125] Ablikim M et al. (BESIII Collab.) Phys. Rev. Lett. 108, 182001 (2012)

[126] Wu J-J et al. Phys. Phys. Rev. Lett. 108, 081803 (2012)

[127] Aceti F et al. Phys. Rev. D 86, 114007 (2012)

[128] Wu X-G et al. Phys. Rev. D 87, 014023 (2013)

[129] Roca L Phys. Rev. D 88, 014045 (2013).

[130] Close F E, Kirk A Phys. Rev. D 91, 114015 (2015)

[131] Sekihara T, Kumano S Phys. Rev. D 92, 034010 (2015)

[132] Sekihara T, Kumano S J. Phys. Soc. Jpn. Conf. Proc. 8, 022006 (2015)

[133] Aceti F, Dias J M, Oset E Eur. Phys. J. A 51, 48 (2015)

[134] Ablikim M et al. (BESIII Collab.) Phys. Rev. D 92, 012007 (2015)

[135] Achasov N N, Kozhevnikov A A, Shestakov G N Phys. Rev. D 92, 036003 (2015)

[136] Albaladejo M, Moussallam B PoS 15, (2015) 057; arXiv: 1510.06626

[137] Wang W Phys. Lett. B 759, 501 (2016)

[138] Albaladejo M et al. JHEP 2017, 10 (2017)

[139] Albaladejo M, Moussallam B Eur.Phys.J. C 77, 508 (2017)

[140] Achasov N N, Shestakov G N Phys. Rev. D 96, 036013 (2017)

[141] Achasov N N, Shestakov G N Phys. Rev. D 96, 016027 (2017)

[142] Sakai S, Oset E, Liang W H Phys. Rev. D 96, 074025 (2017)

[143] Bayar M, Debastiani V R Phys. Lett. B 775, 94 (2017)

[144] Ablikim M et al. (BES Collab.) Phys. Lett. B 607, 243 (2005)

[145] Nakamura K et al. (Particle Data Group) J. Phys. G 37, 075021 (2010)

[146] Ecker G et al. Phys. Lett. B 223, 425 (1989)

[147] Ecker G et al. Nucl. Phys. B 321, 311 (1989)

[148] Birse M C Z. Phys. A 355, 231 (1996)

[149] Bitukov S I et al. (LEPTON-F Collab.) Phys. Lett. B 144, 133 (1984)

[150] Armstrong T A et al. (ABBC Collab.) Phys. Lett. B 146, 273 (1984)

[151] Armstrong T A et al. (WA76 Collab.) Z. Phys. C 34, 23 (1987)

[152] Achasov N N, Kozhevnikov A A Z. Phys. C 48, 121 (1990)

[153] Achasov N N, Kozhevnikov A A Phys. Atom. Nucl. 56, 1261 (1993); Yad. Fiz. 56, 191 (1993)
[154] Achasov N N, Kozhevnikov A A Phys. Rev. D 49, 275 (1994)

[155] Achasov N N, Shestakov G N JETP Lett. 107, 276 (2018); Pis'ma Zh. Eksp. Teor. Fiz. 107, 292 (2018)

[156] del Amo Sanchez P et al. (BaBar Collab.) Phys. Rev. D 83, 052001 (2011)

[157] Aubert B et al. (BaBar Collab.) Phys. Rev. D $\mathbf{7 9}$, 032003 (2009)

[158] Mitchell R E et al. (CLEO Collab.) Phys. Rev. D 79, 072008 (2009)

[159] Naik P et al. (CLEO Collab.) Phys. Rev. D 80, 112004 (2009)

[160] Onyisi P U E et al. (CLEO Collab.) Phys. Rev. D 88, 032009 (2013)

[161] Muramatsu H et al. (CLEO Collab.) Phys. Rev. Lett. 89, 251802 (2002)

[162] Rubin P et al. (CLEO Collab.) Phys. Rev. Lett. 93, 111801 (2004)

[163] Aubert B et al. (BaBar Collab.) Phys. Rev. D 78, 034023 (2008)

[164] Peng $\mathrm{T}$ et al. (Belle Collab.) Phys. Rev. D 89, 091103(R) (2014)

[165] del Amo Sanchez P et al. (BaBar Collab.) Phys. Rev. Lett. 105, 081803 (2010)

[166] Aubert B et al. (BaBar Collab.) Phys. Rev. D $\mathbf{7 2}$, 052008 (2005)

[167] Garmash A et al. (Belle Collab.) Phys. Rev. D 91, 072003 (2015)

[168] Achasov N N, Shestakov G N Phys. Rev. D 96, 091501(R) (2017)

[169] Garmash A et al. (Belle Collab.) Phys. Rev. Lett. 96, 251803 (2006)

[170] Achasov N N, Gubin V V Phys. Lett. B 363, 106 (1995)

[171] Ablikim M et al. (BES Collab.) Phys. Lett. B 607, 243 (2005)

[172] Bonvicini G et al. (CLEO Collab.) Phys. Rev. D 76, 012001 (2007)

[173] Albrow M G, Coughlin T D, Forshaw J R Prog. Part. Nucl. Phys., 65149 (2010)

[174] Kirk A Int. J. Mod. Phys. A 29, 1446001 (2014)

[175] Gutierrez G, Reyes M A Int. J. Mod. Phys. A 29, 1446008 (2014)

[176] Khachatryan V et al. (CMS Collab.) arXiv:1706.08310

[177] Akesson T et al. (AFS Collab.) Nucl. Phys. B 264, 154 (1986)

[178] Au K L, Morgan D, Pennington M R Phys. Rev. D 35, 1633 (1987)

[179] Armstrong T A et al. (WA76 Collab.) Z. Phys. C 51, 351 (1991)

[180] Barberis D et al. (WA102 Collab.) Phys. Lett. B 453 , 316 (1999)

[181] Barberis D et al. (WA102 Collab.) Phys. Lett. B 453, 325 (1999)

[182] Reyes M A et al. (E690 Collab.) Phys. Rev. Lett. 81, 4079 (1998)

[183] Barberis D et al. (WA102 Collab.) Phys. Lett. B 453 , 305 (1999)

[184] Barberis D et al. (WA102 Collab.) Phys. Lett. B 462 , 462 (1999)

[185] Armstrong T A et al. (WA76 Collab.) Z. Phys. C 52, 389 (1991)

[186] Barberis D et al. (WA102 Collab.) Phys. Lett. B 440, 225 (1998)

[187] Barberis D et al. (WA102 Collab.) Phys. Lett. B 413, 
$225(1997)$

[188] Sosa M et al. (E690 Collab.) Phys. Rev. Lett. 83 913, (1999)

[189] Barberis D et al. (WA102 Collab.) Phys. Lett. B 471, 440 (2000)

[190] Barberis D et al. (WA102 Collab.) Phys. Lett. B 474, $423(2000)$

[191] Barberis D et al. (WA102 Collab.) Phys. Lett. B 488, 225 (2000)

[192] Ganguli S N, Roy D P Phys. Rep. 67, 203 (1980)

[193] Alberi G, Goggi G Phys. Rep. 74, 1 (1981)
[194] Streng K H Phys. Lett. B 166, 443 (1986)

[195] Achasov N N, Shestakov G N Phys. Rev. D 97, 054033 (2018)

[196] Sobol A E, Ph.D. Thesis (Protvino-Annecy-le-Vienx, 2001)

[197] Achasov N N and Shestakov G N Phys. Rev. D 58, 054011 (1998)

[198] Ablikim M et al. (BESIII Collab.) Phys. Rev. Lett. 121, 022001 (2018) 